\title{
WestVirginiaUniversity
}

THE RESEARCH REPOSITORY @ WVU

Graduate Theses, Dissertations, and Problem Reports

2003

\section{Finite element analysis of cell subjected to compressive loading}

Vidhyashankar Venkatesan

West Virginia University

Follow this and additional works at: https://researchrepository.wvu.edu/etd

\section{Recommended Citation}

Venkatesan, Vidhyashankar, "Finite element analysis of cell subjected to compressive loading" (2003). Graduate Theses, Dissertations, and Problem Reports. 1302.

https://researchrepository.wvu.edu/etd/1302

This Thesis is protected by copyright and/or related rights. It has been brought to you by the The Research Repository @ WVU with permission from the rights-holder(s). You are free to use this Thesis in any way that is permitted by the copyright and related rights legislation that applies to your use. For other uses you must obtain permission from the rights-holder(s) directly, unless additional rights are indicated by a Creative Commons license in the record and/ or on the work itself. This Thesis has been accepted for inclusion in WVU Graduate Theses, Dissertations, and Problem Reports collection by an authorized administrator of The Research Repository @ WVU. For more information, please contact researchrepository@mail.wvu.edu. 


\title{
Finite Element Analysis of cell subjected to compressive loading
}

\author{
Vidhyashankar Venkatesan \\ Thesis submitted to the \\ College of Engineering and Mineral Resources \\ at West Virginia University \\ in partial fulfillment of the requirements \\ for the degree of \\ Master of Science \\ in \\ Mechanical Engineering \\ Nilay Mukherjee, Ph.D., Chair \\ Ever J. Barbero, Ph.D., \\ Timothy L. Norman, Ph.D.,
}

Department of Mechanical and Aerospace Engineering

Morgantown, West Virginia

2002

Keywords: Cell mechanics, Buckling analysis, ABAQUS, Finite element. 


\title{
Abstract \\ Finite Element Analysis of cell subjected to compressive loading
}

\author{
Vidhyashankar Venkatesan
}

A 3D finite element model was built to study the mechanical response of the cell to load. The model included major structural components of a cell like cytoskeletal elements, cell membrane and the presence of internal fluid pressure. The cell was considered as a pressurized, fluid filled bag where inflation of the cell membrane is resisted by the cytoskeletal elements. The behavior of the cell model with microtubules prone to buckling and stabilized by actin filaments was also studied.

A point load was applied to a microtubule in a cell model with there buckling effect taken into consideration. The microtubule started to buckle at around 54.5 PN. A parametric analysis was performed to study the effect of each structural component on overall stiffness of cell measured in terms of modulus. This study showed microtubules and internal fluid pressure were important elements in determining the overall stiffness of the cell. 
This thesis is dedicated to B apu and M otima 


\section{Acknowledgement}

I would first like to thank God for being with me at all time and giving me the strength.

I would like to thank Dr. Nilay Mukherjee, for giving me the opportunity to work under him as a Graduate Research Assistant and finish my thesis under his guidance. He has been a major source for the knowledge I have gained. I would also like to thank Dr. Timothy L Norman and Dr. Ever J Barbero for serving on my committee and providing with valuable suggestions.

Thanks to Ganesh Thyagarajan and Madhavi Ayyalasomayajula for being of constant help during the project. I would also like to thank my cousin Shyam Davey for being a source of encouragement and support through out my stay away from home.

I would also like to thank all my friends for being there when I needed them and for all the fun that we had during our study away from home. Special thanks to Padha, Michael, and all my roommates for being a source of encouragement to me.

Finally I would like to thank my family, especially my grand mother, for their encouragement and prayers. 


\section{TABLE OF CONTENTS}

Chapter 1: Introduction .......................................................................................... 1

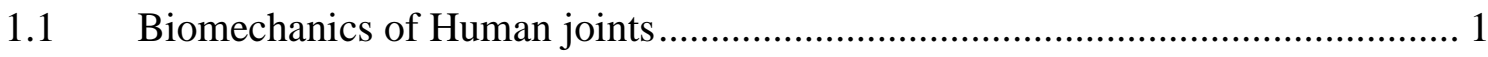

1.2 Articular cartilage structure, shape and function ........................................... 4

1.3 Bone- Structure, shape and function .................................................. 6

1.4 Mechanical loading affect biological behavior of tissue ............................... 7

1.5 Structural elements of cell ................................................................... 9

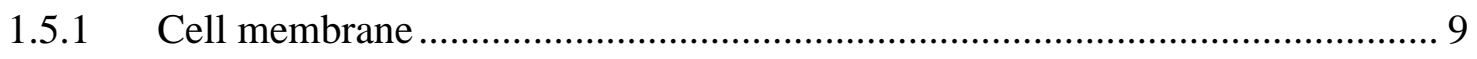

1.5.2 Cytoskeletal elements ...................................................................... 9

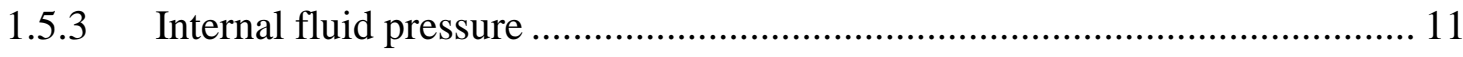

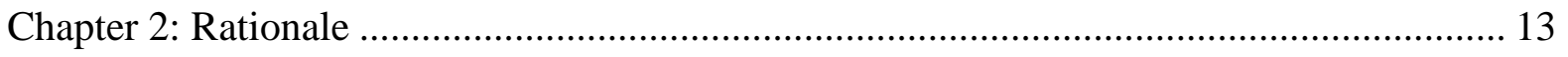

Chapter 3: 3D Finite Element Model of a Cell incorporating internal fluid pressure ........... 16

3.1 Description of cell model.................................................................... 16

3.2 Description of the model generation program .......................................... 18

3.3 Bulk modulus extraction program .......................................................... 24

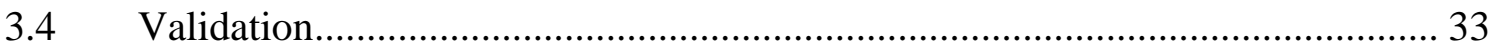

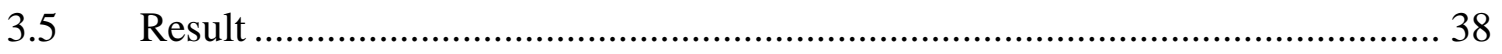


3.6 Discussion

Chapter 4: Buckling Behavior of the microtubule 40

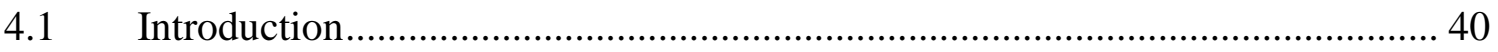

4.2 Buckling - a review ........................................................................... 41

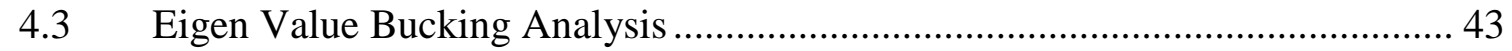

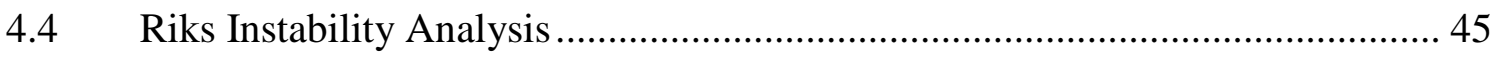

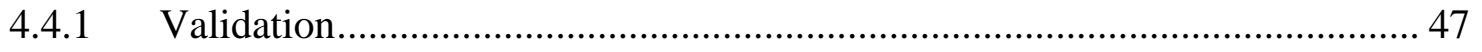

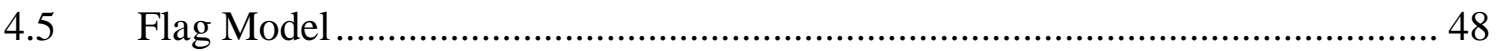

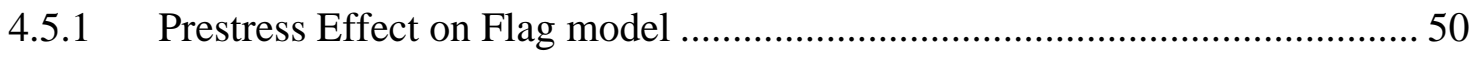

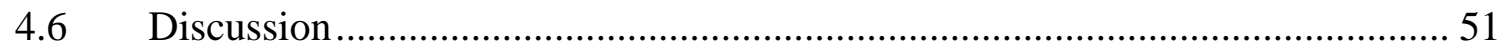

Chapter 5: Construction of a layered Finite Element Model of a Cell ................................ 53

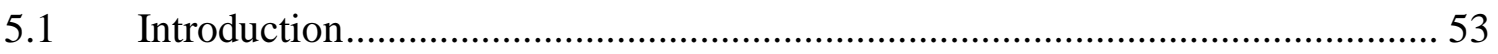

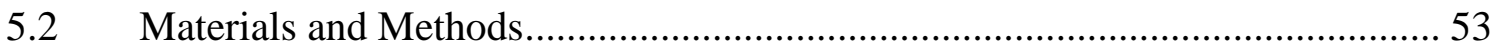

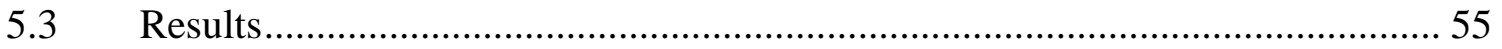

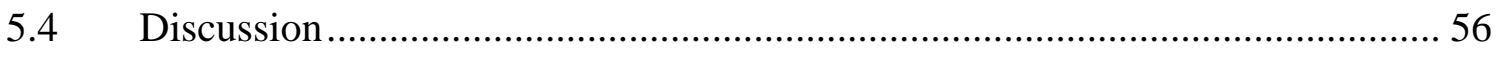


Chapter 6 -Buckling of microtubules in a 3D cell model.

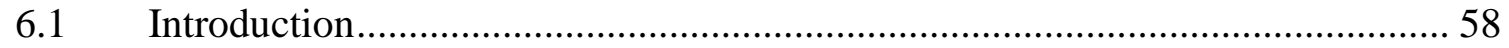

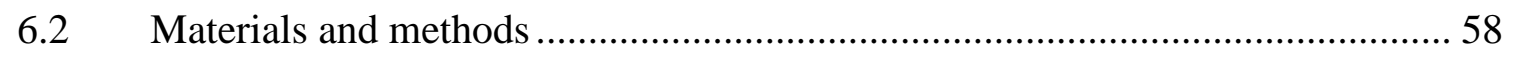

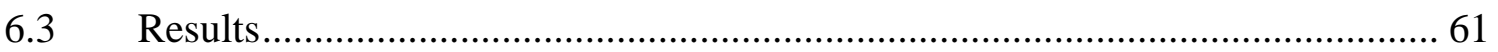

Chapter-7 :Uniaxial loading of 3D Finite Element Model of cell ........................................... 68

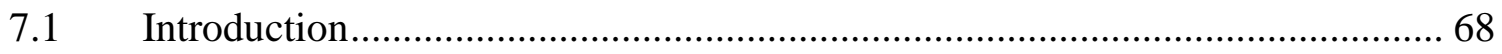

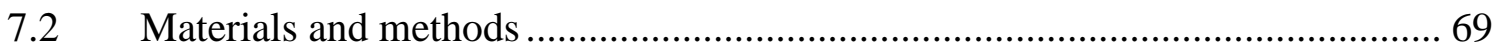

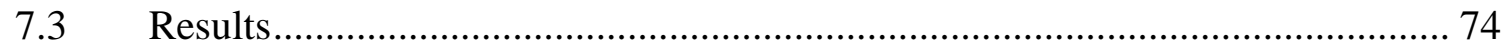

Chapter 8 : Conclusion and Future Studies..................................................................... 79

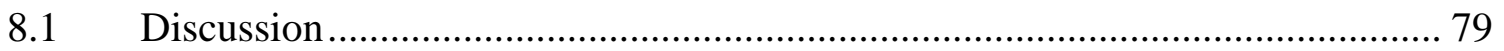

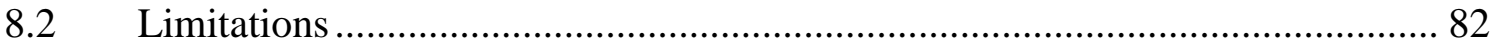

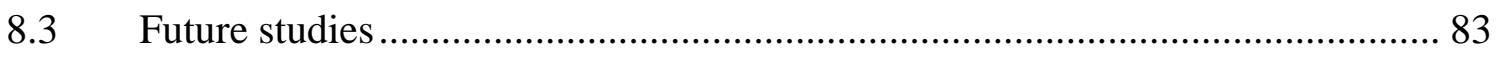

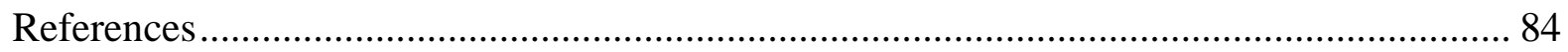

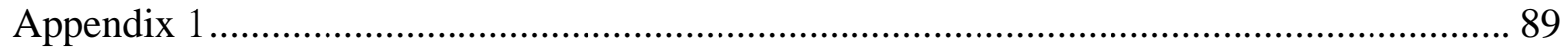

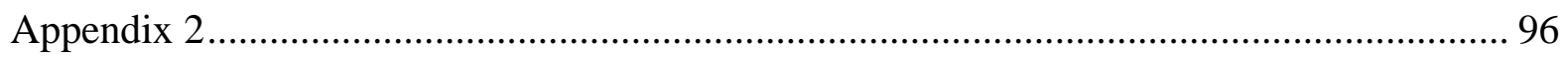


Appendix 3 ......

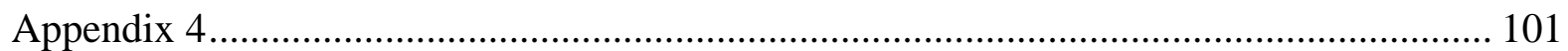

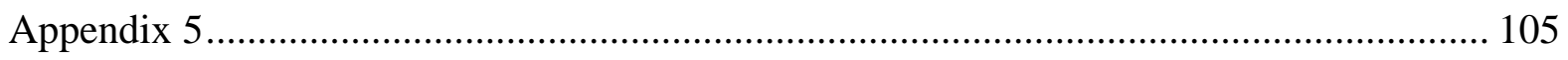

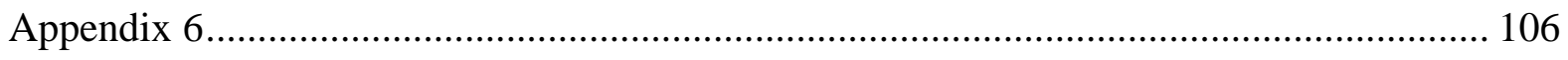

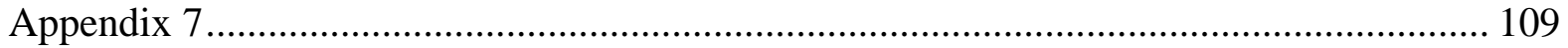

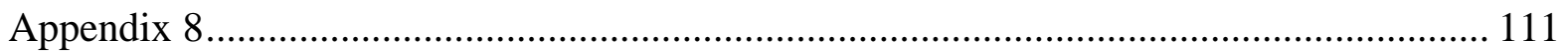

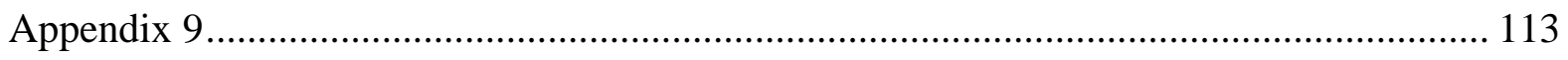




\section{LIST OF FIGURES}

Figure $1 \mathrm{~A}$ biomechanics of a hip in single legged stance................................................ 1

Figure 2 Biomechanics of a leg when climbing the stairs. ................................................. 1

Figure 3 Structure of cartilage tissue. ......................................................................... 5

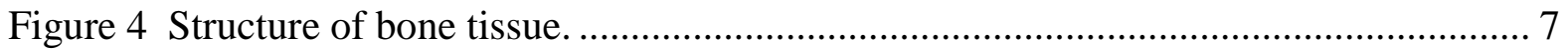

Figure 5 Structure of membrane elements ............................................................ 9

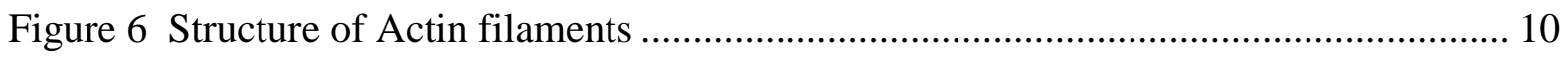

Figure 7 Structure of microtubule elements........................................................... 11

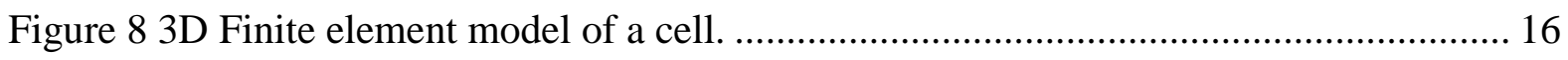

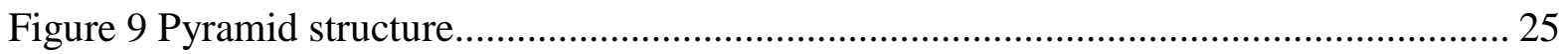

Figure 10 Procedure for cytoskeletal cell model validation. .......................................... 34

Figure 11 Validation of Finite element model with closed form solution ............................ 36

Figure 12 Accuracy of continuum FE spherical model with a closed form solution. ............ 37

Figure 13 Accuracy of continuum FE spherical model with a closed form solution. ............ 37

Figure 14 Effect of Young's modulus of cytoskeletons on the bulk modulus of cell. .......... 38

Figure 15 Effect of number of cytoskeletal elements on the bulk modulus of cell. .............. 38

Figure 16 Effect of internal fluid pressure on the bulk modulus of the cell. ....................... 39

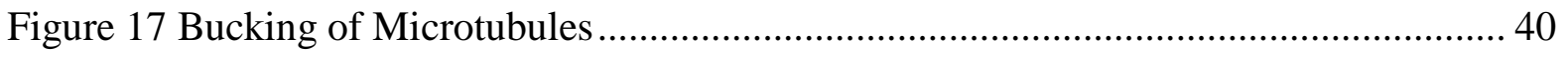

Figure 18 columns with pin-ended boundary condition. ........................................... 42 


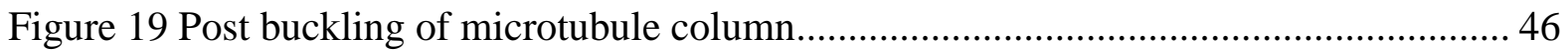

Figure 20 comparision of post buckling results of ABAQUS with elastica solution............ 48

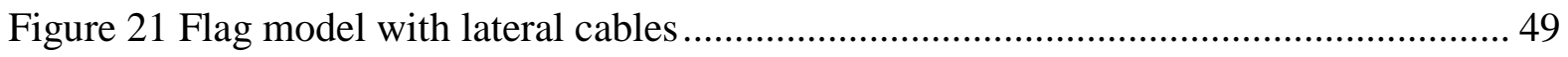

Figure 22 comparision of postubuckling of flag model with rope like lateral elements Vs rod

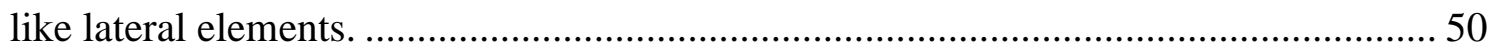

Figure 23 Effect of prestress on the pre and post buckling behavior of the flag model ......... 51

Figure 24 Schematic representation of layered addition of structural elements ..................... 54

Figure 25 3D finite element model of a cell ...................................................................... 59

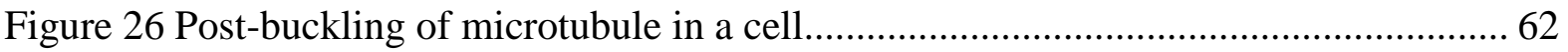

Figure 27 Effect of pre-stress of actin filaments on the post-buckling stiffness of the cell. .. 63

Figure 28 Post-buckling behavior of the neighboring microtubules ...................................... 64

Figure 29 Action at a distance- a pulling load applied at a point. .......................................... 65

Figure 30 Action at a distance - microtubule in a cell subjected to compressive point load. 66

Figure 31 Goodier's model of spherical inclusion inside a infinite medium.......................... 69

Figure 32 load required in goodier's model equivalent to the load applied to cell model .... 71

Figure 33 Schematic representation of finding the equivalent young's modulus for uniaxial

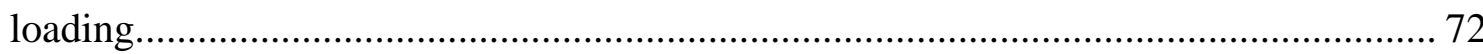

Figure 34 Closeness of Fit between the cell model Vs the goodier's closed form solution... 73 Figure 35 Variation of dimensionless equivalent young's modulus of the 3D cell model with

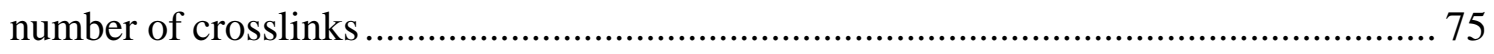

Figure 36 Variation of dimensionless equivalent young's modulus of 3D cell model with

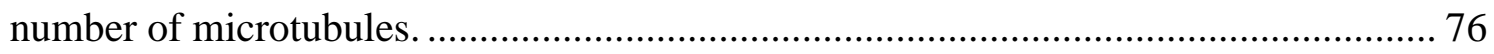


Figure 37 A variation of dimensionless young's modulus of cell (E/E0) with the variation of

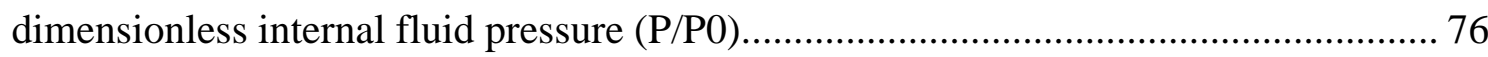

Figure 38 Variation of dimensionless young's modulus of cell (E/E0) with the variation of

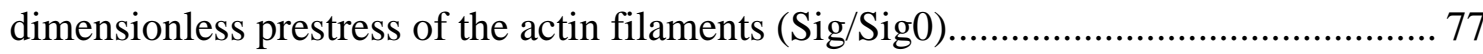




\section{LIST OF TABLES}

Table 1 Accuracy of the validation of cytoskeletal model with a closed form solution......... 34

Table 2 Effective length for a column with different end conditions............................... 41

Table 3 Comparison of ABAQUS Eigen value results with theoretical calculation............. 44

Table 4 Effect of dead load on the critical buckling load.............................................. 45

Table 5 The stress on structural elements layered addition of each component.................... 56 


\section{Chapter 1: Introduction}

\subsection{Biomechanics of Human joints}

Man is involved in various kinds of physical activities everyday like walking, running, jumping, lifting, etc., Biomechanics is the science that estimates the mechanical forces and moments on various tissue in our body as a result of these activities.

Biomechanics uses the principal of kinetics to study the various kinds of forces and their reactions on various orthopedic joints like knee, spine, shoulder, foot, etc., and also tissues like bone, cartilage, ligament, tendons etc., Various activities result in different kinds of forces acting on joints like push, pull, twist etc., These forces and reactions are represented in terms of vector components that can be broken down into normal and shear force.

The focus on the hip joint is shown in Fig-1 [1].
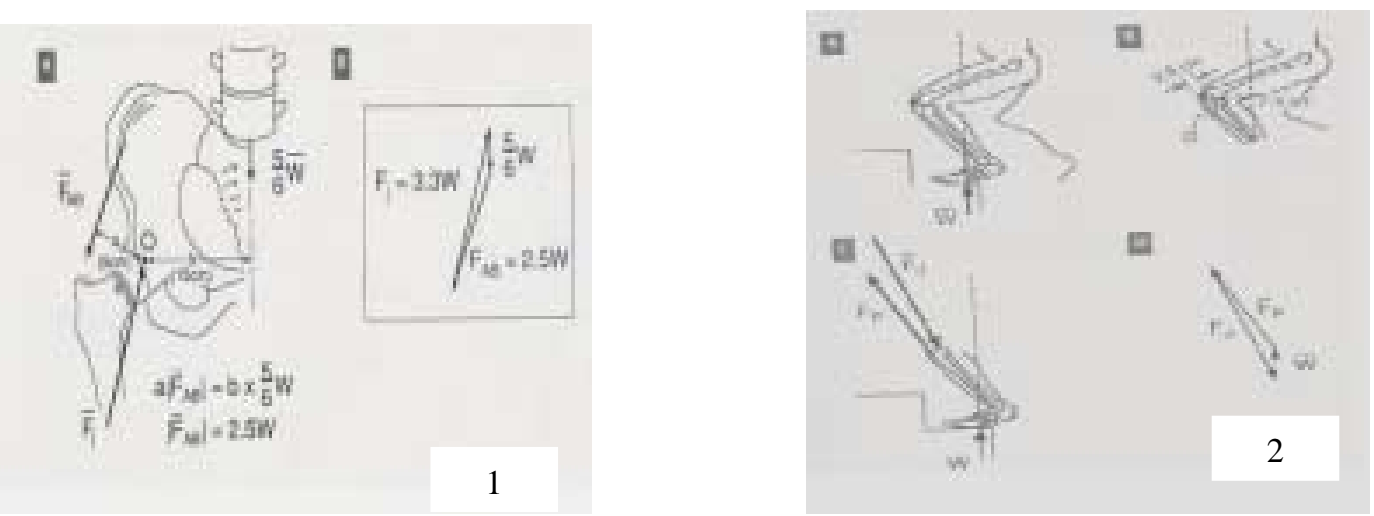

Figure 1 A biomechanics of a hip in single legged stance.

Figure1 from Buckwalter et al., [1] $\mathrm{F}_{\mathrm{AB}}$ is the force applied by the abductor muscles on the pelvis can be seen, the joint reaction force can be as high as 3.3 times body weight $\mathrm{W}$.

Figure 2 Biomechanics of a leg when climbing the stairs.

$\mathrm{W}$ is the reaction force acting on single feet due to his self-weight. The resultant forces on the knee joint are given by the vector forces $F_{P}$ and $F_{j}$, which is represented as a vector diagram. 
If $\mathrm{W}$ is the weight of the person, and if the weight of the left leg is assumed to be $1 / 6$ times $\mathrm{W}$, then the balancing force at the abductor muscle is found to be 2.5 times the weight of person. Hence the reaction force at the hip as a result of the person's body weight is found to be 3.3 times weight of the person.

Similarly the biomechanics of person climbing stairs is shown in Fig-2[1]. If $\mathrm{W}$ is the person's weight, the reaction force on the foot balances it. By using biomechanics principle the magnitude of force at the patellar tendon is found to be 3 times the weight of body and the joint reaction force at the knee is found to be 3.5 times the body weight.

Similar principles are applied to various other orthopedic joints involved in different physical activities like forces on the shoulder of a man lifting weight, forces on the spine etc., These forces acting also depend on the kind of day to day activities a person is involved, and loading can also be of different types like cyclic in nature when walking, running or continuous in nature when standing still etc., Thus large forces are applied to human joints during everyday activities.

These large forces are applied for millions of cycles of loading over the lifetime of a person. An average number of steps walked by person can be 7904(+/- 2534) [2], which approximates to around $3.9(+/-1.2)$ miles per day. Therefore for a person having an average life span of 65 years would walk on an average 18.75 million steps. This is just in case of normal walking, while the other activities would involve sitting, running, jumping, lifting weights, etc.,

This means that the body copes with large forces and repetitive cycles of loading. It does this by constantly repairing and regenerating the tissue that is damaged by loading. This process is called remodeling. Not only does the human joints withstand these large cycles of 
loading but they are also responsible for remodeling the tissue according to the kind of physical activities a person is involved. The strength of the joints and tissues are different for people involved in different activities like athletes, volleyball players, swimmers, astronauts, tennis players, etc., It is well known fact that the strength of the joint and tissue depend on the mechanical forces acting. In case of long-term space flight, the reduced mechanical forces results in reduction in bone mass[3]. Studies show that the bone and mineral metabolism is limited in case of humans working in micro gravity [4]. On the opposite end the bone mass was found to be higher in case of professional football players [5] than the people involved in normal day to day activities. Bone mineral densities were also found to be different in case people involved in different athletic activities e.g. karate athletes, judo athletes had higher bone mineral densities (BMD) than that of water polo players[6]. Also the BMD of axial skeleton and limbs were higher in case of volleyball players than in case of control subjects. Thus the body possesses a mechanism to measure the loads applied to different tissues and respond to these loads by appropriately remodeling the tissue so that it can withstand these loads.

It is well known that cells are responsible for making up the Extra Cellular Matrix (ECM) that further govern the tissue shape and function The cells are responsible for building the tissue material. At cellular level the tissue surrounding the cell is called ECM. The major tissues that bear mechanical loadings are bones, cartilage, muscles, tendon, etc., The major kind of loading in tissue like bone and cartilage is compressive. The cell in bone is called osteons and in case of cartilage is called chondrocytes. The cells are responsible for continuous breakdown and rebuilding of the ECM in response to the type of activity a person 
is involved. The exact mechanism by which this works at the cellular level is poorly understood.

\subsection{Articular cartilage structure, shape and function}

Articular cartilage is a tissue that acts as an articulating surface between diarthrodial joints[7] [8]. It provides the necessary lubrication and resistance against wear for repetitive articulation. Cartilage primarily consists of specialized cells called chondrocytes that make up the extra cellular matrix (ECM) that surrounds the cells. The major structural components that make up the ECM are water, proteoglycans and collagens that combine in a unique manner to give cartilage its unique property [7] [8]

Articular cartilage is primarily divided into four zones based on the structure and composition of the ECM components through the depth of the tissue. The first zone called the superficial zone and forms the gliding surface. Here the collagen fibrils are arranged parallel to the articulating surface in order to withstand high shear forces that this layer is subjected to. The second zone called the middle zone has collagen arranged in a random fashion with more rounded chondrocytes making it good for resisting compression. The third zone called the deep zone contains collagen-arranged perpendicular to the surface thus anchoring cartilage to bone. This zone has the highest concentration of the proteoglycans and lowest water content. The last zone called the zone of calcified cartilage separates the cartilage from subchondral bone[1]. The chondrocytes derive from the mesenchymal stem cells differentiate during skeletal morphogenesis to form chondrocytes, which then secrete cartilaginous tissue around the cells. 
Figure 3 Structure of cartilage tissue.

The rounded elements are chondrocytes distributed in the cartilage tissue.

Figure from

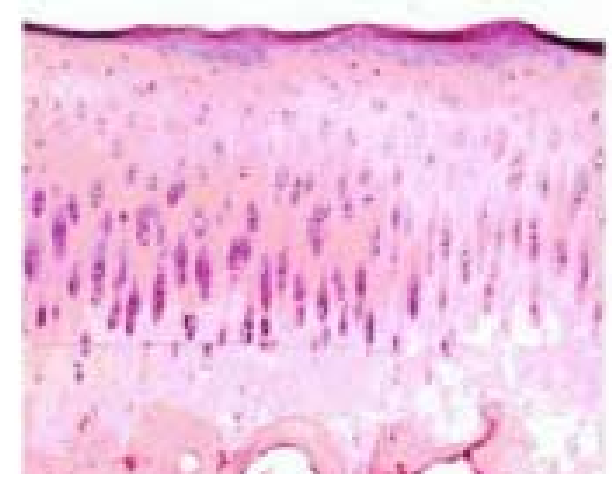

Dahweb.engr.ucdavis.edu/dahweb/126site/bone.htm

They occupy less than $10 \%$ of the total tissue volume [1]. Chondrocytes respond to various environmental stimuli to maintain the structure and function of the ECM. The environmental stimuli can be of various kinds like growth factors, interleukins, mechanical loads etc.,

The other structural components are water that make up to $65 \%$ to $80 \%$ of the total weight, collagen that make up to 10 to $20 \%$ of the total weight and proteoglycans that contribute less than 5\% [1]. Water can move through the ECM with high frictional resistance due to small pore size [8]. The frictional resistance is one of the mechanism by which cartilage supports high loads. Collagens form a triple-helical structure provides cartilage with shear and tensile property [1]. They have different orientation along the depth of the tissue providing different function as explained earlier[1]. The biomechanical property of the articular cartilage is considered to be a contribution of different mechanical features like of the constitiuents of the tissue. This gives the cartilage its unique structure and property under normal functional condition.

Thus the cells control the micro-structure of cartilage that helps it to perform its mechanical function. 


\subsection{Bone- Structure, shape and function}

Bone is a major structural component of a human body. The bone tissue has a unique structure and function, which provides strength and low weight[1]. It is structurally organized into two types. One is called the cortical bone, which is dense, and tabecular bone, which is porous. Trabecular bone primarily takes up compressive loading, while the cortical bone takes up torsion and bending apart from the compressive loading[1]. The cells that make up the bone tissue are osteoclasts and osteoblasts. Osteoblasts also called bone-building cells make up the bone tissue. Osteoclasts are bone resorptive cells characterized by its large size and multiple nuclei and are responsible for breaking down the bone tissue at regular intervals. Osteoblasts and osteoclasts work together in remodeling process of the cell [1]. Osteoclasts continuously break down the bone matrix and osteoblast rebuild the bone matrix at the site where osteroclasts break down the tissue. This remodeling process occurs along the length of the bone. The remodeling process depends on various factors; mechanical stress is one of the major factors. The rate of remodeling eventually declines during the life span at a rate of 2 to $5 \%$ per year [1]. The breakdown and rebuilding occurs at constantly at specific cycles.

Remodeling repairs damaged bony tissue as a result of loading and makes the bone strongest in the direction of principle stresses. For example a finite element analysis of the femur revealed that the tabecular bone is laid down exactly along the lines joining the principal directions at each point in the bone.

The unique property of bone is to change its structural property in response to its mechanical environment. Increased bone mineral density after heavy exercise or a decrease 
in density as a result of age related activity gives a need for a better understanding of the bone remodeling process and response to its mechanical environment.

Figure 4 Structure of bone tissue.

The round dark regions are osteons distributed in a bone tissue.

Figure from

Dahweb.engr.ucdavis.edu/dahweb/126site/bone.htm

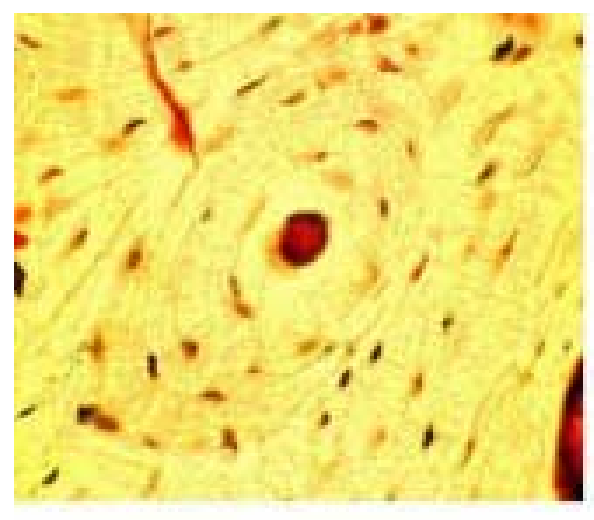

\subsection{Mechanical loading affect biological behavior of tissue}

Compressive loading is intrinsic to certain tissues in our body like cartilage and bone[8]. Cartilage is built and maintained by specialized cells called chondrocytes [1] [9]. In situ experiments in cartilage suggest that chondrocytes can undergo significant deformation due to compressive loading on the tissue[10, 11]. In situ and isolated cell experiments have concluded that shuch cells are quite resilient to compressive loading, aspiration etc., and exhibit moduli in the range of 0.6 to $2 \mathrm{KPa}[12,13]$.

It is generally accepted that the cells in bone and cartilage sense the mechanical stresses/strains imposed on the tissue and respond by remodeling the tissue so that the tissue can efficiently perform its mechanical function $[10,14,15]$. Chondrocytes in vivo and embedded in agrose gel, show a turnover, change in metabolism and biosynthesis of the extra-cellular matrix. Studies show that a reduction in proteoglycan synthesis around chondrocyte subjected to a constant static loading.[7, 16]. Dynamic strains of various frequencies have shown to increase the amount of proteoglycan synthesis as well as 
pericellular matrix stimulation [16] [7]. The mechanical strains also affect the aggrecan synthesis relative to the direction and amount of applied load [17]. Studies also show that the stiffness of the ECM increased by 6 fold with dynamic load applied over a period of time [18]. Isolated cells on a culture dish respond to mechanical stimuli by reorganizing their cytoskeletal architecture [9]. The mechanisms by which the cells sense these mechanical signals have not been well understood. A possible hypothesis could be that cells reorganize their cytoskeletal structure to either tune in or out certain frequencies of oscillatory loads. It would be of interest to know how mechanical stimuli is transmitted through the cytoskeletal framework of a cell, and whether some structural features are more conducive for this purpose than others. A finite element model of the cell based on its cytoskeletal architecture would be the starting point of such investigation. The major structural elements that are responsible for resisting the mechanical loads include cell membrane, cytoskeletal elements (actins filaments and microtubules). Internal fluid pressure also play a role in resisting compressive loads. The mechanical and physical properties play a important role in resisting mechanical loads which needs to be discussed in detail. 


\subsection{Structural elements of cell}

\subsubsection{Cell membrane}

Structurally the cell membrane forms the outer wall for the cell. It resists tensile loading, primarily from internal fluid pressure. It has a bilipid layer as shown in fig 5 . The young's modulus of the cell membrane was found to be $0.1 \mathrm{Mpa}[19]$. The thickness of the cell membrane is about $5 \mathrm{~nm}[20]$.
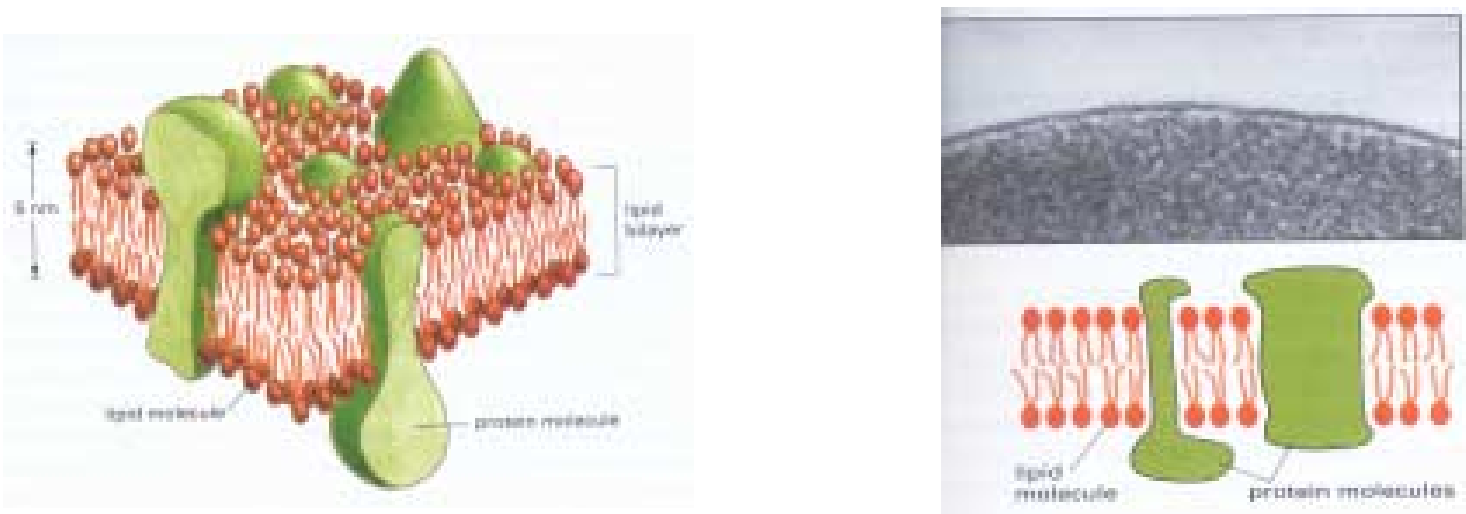

Figure 5 Structure of membrane elements

The bilipid structure of membrane is 5 nano-meters in thickness. Figure from Alberts et al., [20]

\subsubsection{Cytoskeletal elements}

\section{Actin}

Actin filaments are one of the major structural elements of a cell. They form an extensive network as well as the long rope like structure shown in Fig-6. They are involved in structural stability of cell as well as involved in cell motility. They actin fibers are $8 \mathrm{~nm}$ in diameter [21]. It is found that the actin are physically attached to the extra cellular matrix through special complexes called integrins. The flexural rigidity of the actin is found to be 7.3E-26 $\mathrm{Nm}^{2}$ [22] from which we arrive at the young's modulus to be $363 \mathrm{MPa}$. 

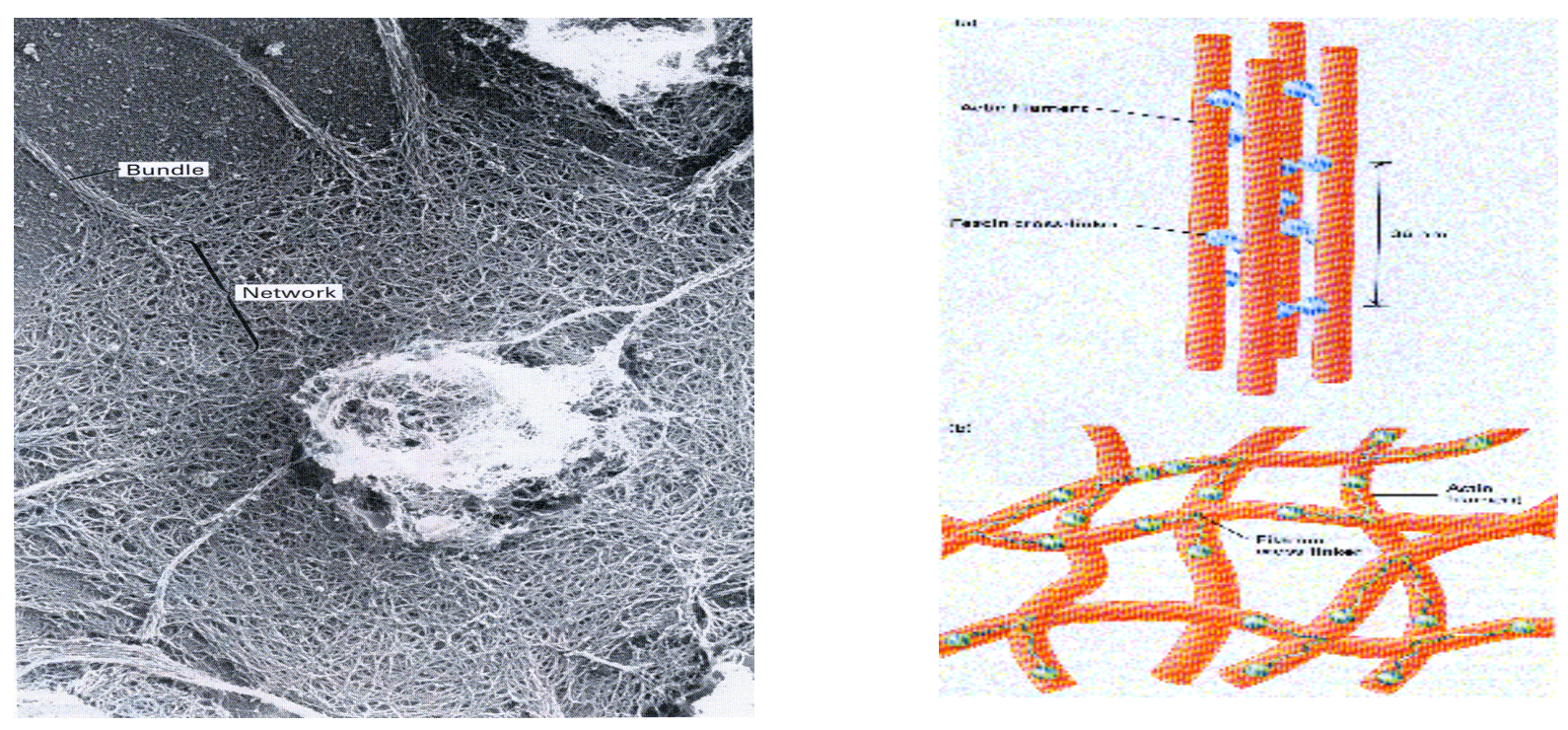

Figure 6 Structure of Actin filaments

They form extensive network as well as long bundles of ropes. They are responsible for taking up tensile loads in a cell. The actin fibres are $8 \mathrm{~nm}$ in diameter. Figure from Alberts et al., [20]

\section{Microtubules}

Microtubules are hollow pipe like elements that resist compression in a cell as shown in fig-7. They are made of 13 different subunits called tubulin. The external diameter of microtubule is $25 \mathrm{~nm}$ and the thickness is $2.7 \mathrm{~nm}$ [21]. The moment of inertia I is calculated from the cross-sectional area to be $1.628 \mathrm{E}-32 \mathrm{~m}^{4}$. The flexural rigidity (EI) of microtubules was found to be $2.1 \mathrm{E}-23 \mathrm{Nm}^{2}$ [22] which is about 1000 times stiffer in bending than actin filaments. There fore the Young's modulus is calculated by dividing the flexural rigidity of the microtubules by its moment of inertia. The Young's modulus was found to be $2.1 \mathrm{E}-$ 23/1.6E-32 = 1.2 GPa. Microtubules are known to take up compressive loads in a cell. 
Figure 7 Structure of microtubule elements.

Microtubule form a hollow columnar shape make up of 13 units of tubulin monomers. Figure from Alberts et al., [20]

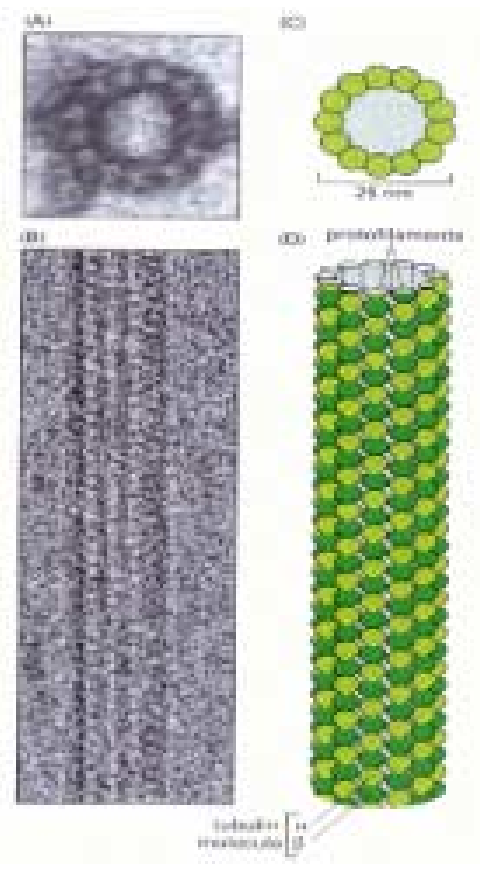

\subsubsection{Internal fluid pressure}

The fluid inside a cell causes induces tensile stress on the cell wall [23] [24]. Osmotic pressure difference between inside the cell and its outer environment plays a role in the fluid flow between the cell and its environment. When the osmotic concentration of the outside medium is increased, it causes the fluid to flow from inside the cell to outside there by decreasing the internal fluid pressure [24] This osmotic pressure difference balances the turgor pressure in a cell. This is given by the formula

$\Delta \mathrm{P}=\Delta \Pi=-\left(\Pi_{\mathrm{e}}-\Pi_{\mathrm{I}}\right)[24]$

where

$\Delta \mathrm{P}$ turgor pressure

$\Delta \Pi$ - osmotic pressure difference between outside the cell and inside.

Also the following relation gives the relation between the volume of the cell and its osmotic concentration. 


$$
V=\frac{\Pi_{e \Delta p=0}\left(V_{\Delta p=0}-b\right)}{\Delta p-\Pi_{e}}+b \quad \text { [24] }
$$

Where $\mathrm{V}$ is cell volume, $\mathrm{b}$ is non-osmotic volume, $\Pi_{\mathrm{e}}$ is external osmotic pressure. This shows that the internal fluid pressure is one of the component responsible for internal volumetric expansion of the cell and further resist compressive loads. 


\section{Chapter 2: Rationale}

Induvidual structural elements of a cell combine and organize to form a architecture that can resist different kinds of loadings. The cytoskeletal architecture of the cells is known to change depending on the shape and dimensionality (2D-vs 3D) of the substrate on which they are grown[25, 26]. Further, these differences in cytoskeletal organization have been linked to differences in behavior or phenotype expressed by the cells and these influences have been shown to be independent of biochemical stimuli [9, 27-29]. One would expect that differences in cytoskeletal structure of a cell would be reflected in the gross mechanical properties of a cell such as modulus of elasticity and anisotropy ratio[25, 30]. Interestingly, it has already been shown that chondrocytes from osteoarthritic cartilage have significantly different material properties than chondrocytes from normal cartilage[31]. Could these be linked to differences in cytoskeletal structure of the cells? If so, the differences in cytoskeletal structure could then be used as mechanical markers of cellular phenotype and could have important implications in diagnosing disease or predicting cell behavior. A finite element model can provide a theoretical basis for such ideas and can provide clues for how much one can expect changes in gross material properties of a cell when its cytoskeletal structure changes by a certain amount. These in turn can lead to well-defined experiments that can then aim to measure such changes.

The second mechanism speculates that the microtubules and actin/intermediate filaments form a "tensegrity" structure in which the microtubules are in pre-compression and 
the actin/intermediate filaments are in pre-tension $[30,32]$. This theory is supported by experimental evidence based on changes in cell shape and load transmission to substrate on which the cell is grown when pharmaceutical agents that disrupt specific cytoskeletal structures are used or when portions of the cell are physically manipulated. While the specifics of the tensegrity model are under debate[32], there is a consensus that a combination of pre-tension/ pre-compression probably exists in the cytoskeletal architecture. We used this feature in our model.

There exist evidence that microtubules are the compression bearing elements in a cell $[30,33]$. In neuronal cells, when the microtubule elements were dissolved, the cell changed its mechanical properties in a way that indicated that the microtubules probably resisted compression [33-35]. In other experiments, the transfer of force to a stretchable membrane on which the cells were grown was measured when the microtubule network was dissolved. These also indicated that the microtubules were in compression [30, 33, 35]. Direct visualization experiments show that microtubules can buckle inside a cell ostensibly due to compressive loading [30]. Microtubules have a very high slenderness ratio (Length $=7.5 \mu \mathrm{m}$, diameter $=25 \mathrm{~nm}$, thus slenderness ratio $=300$ ), thus making them prone to buckling. However, the inter connections with other cytoskeletal elements would prevent a catastrophic failure of the microtubules in buckling. We thus incorporated buckling of microtubules and modeled the post buckling behavior of the microtubules in our model.

The third mechanism postulates that the internal fluid pressure of the cell tries to expand the cell and the cell membrane $[23,24,36,37]$. Hence the cell behaves like a blownup balloon, which can resist compression. Osmotic experiments confirm the presence of internal fluid pressure in cells, wherein cells swell or shrink when the molarity of the 
extracellular fluid is changed $[36,38]$ Unpublished results in our laboratory show that when the cell membrane of certain cells embedded in agarose gel is ruptured with a micropipette, they undergo shrinking in size that would be expected to accompany depressurization. We used the internal fluid pressure of cells in our model.

We thus propose the following model of the cell: In the resting state of the cell, the microtubules should be in compression, the actin should be in tension, the cell membrane should be in tension and there should exist an internal fluid pressure. All of these features would collectively resist external loading. Upon external loading, the microtubules would be prone to buckling and the actin filaments will serve to stabilize the microtubules against catastrophic buckling. 


\section{Chapter 3: 3D Finite Element Model of a Cell incorporating internal fluid pressure}

\subsection{Description of cell model}

A 3D finite element model of cell is created in ABAQUS (Habbit, Karlsson and Sorensen Inc, Pawtucket, RI). The Cell is modeled as a fluid filled membrane sphere of radius 7.5 microns. The membrane is considered as fully permeable. The membrane was held in place by the cytoskeletal elements (actin filaments) emanating from the center of the cell. This prevents the cell from freely blowing up due to intra-fluid pressure. The internal fluid pressure causes the membrane as well as cytoskeletal elements to be in pre-tension. Only $1 / 8$ of cell is modeled considering symmetric conditions. A representative model is shown in fig8. The membrane and cytoskeletal elements are considered to be linear, elastic materials. The cytoskeletal elements in the model are the 2 noded Beam elements in 3-D space (B31), 3noded and 4-noded membrane elements (element type M3D3 and M3D4) were used to model the cell membrane.

Figure 8 3D Finite element model of a cell.

The model Consisits of cell membrane, cytoskeletal elements emanating from the center to the cell membrane. Internal fluid pressure blows up the model causing the cytoskeketal elements to be in pre-tension.

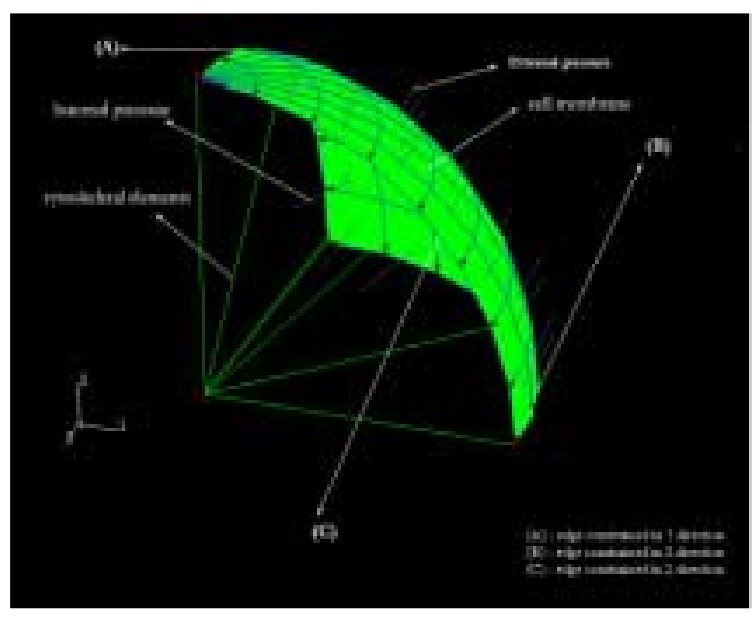


The cytoskeletal elements (truss element- element type T3D2) emanating from the center to the cell membrane are modeled with actin properties and cross-section. This model is now subjected to a internal fluid pressure of $0.05 \mathrm{MPa}$. This caused the whole model to be in tension. Now a compressive load of $0.025 \mathrm{MPa}$ is applied to the membrane surface from the outer surface of membrane. This causes a reduction in cell volume.

\section{Material Properties and Dimensions}

Cell membrane

$$
\begin{aligned}
& \text { Thickness } \quad-0.1 \text { microns.[21] } \\
& \text { Young's modulus - 0.1 Mpa.[19] }
\end{aligned}
$$

Actin

$$
\begin{aligned}
& \text { Diameter } \quad-8 \mathrm{~nm} \mathrm{[21]} \\
& \text { Young's modulus - } 0.363 \mathrm{GPa}[22]
\end{aligned}
$$

Microtubule:

$$
\begin{aligned}
& \text { Diameter } \quad-25 \mathrm{~nm}[21] \\
& \text { Thickness } \quad-2.7 \mathrm{~nm}[21] \\
& \text { Young's modulus }-1.2 \mathrm{Gpa}[22]
\end{aligned}
$$

A FORTRAN (Fortran for Unix Alpha System) code is written to generate a ABAQUS input file representing the model described above. An ABAQUS subroutine is written in FORTRAN to extract the deformed nodal coordinates and then calculate the change in volume of the cell for the applied load to the cell model. The volumetric stiffness of the cell is calculated as Bulk-modulus $(\mathrm{K})=\mathrm{P} /(\Delta \mathrm{V} / \mathrm{V})$. The Cytoskeletal model is validated with a 3D continuum model, and a Closed Form Elasticity solution by Goodier [39] 


\subsection{Description of the model generation program}

A FORTRAN program was written to create an ABAQUS input file representing the 3-D model as shown in fig-8. The program listed in Appendix-1, is described in the Flowchart. First depending on the number of elements required along each edge of the curve and the radius of the sphere, the nodes are generated at the outer surface and at every $1 / 10^{\text {th }}$ distance along the radius of the sphere. The step involved in generating nodes is described in flowchart- B. A model calculation for generation a node in spherical coordinate is shown in appendix-2. The nodes at the outer surface are connected by membrane elements (3-noded \& 4-noded). The step involved in generating the membrane elements is described in flowchartC.

The nodes in between the outer surface and the center of the sphere are connected in a radial direction starting from the center of sphere to the outer surface. Beam (B31) elements with Microtubule properties and Cross-section are used. The steps involved in generating radial elements are described in flowchart-D. The nodes between outer surface and center of sphere are inter-connected across each other at various levels along the radial direction. Truss elements (T3D2) with Actin filament material properties and Cross-section are used with Rope like (No-Compression Option in ABAQUS (Habbit, Karlsson and Sorensen Inc, Pawtucket, RI)) condition since actin can take only tensile loads. The steps involved to generate the cross-link elements are described in flowchart-E. 


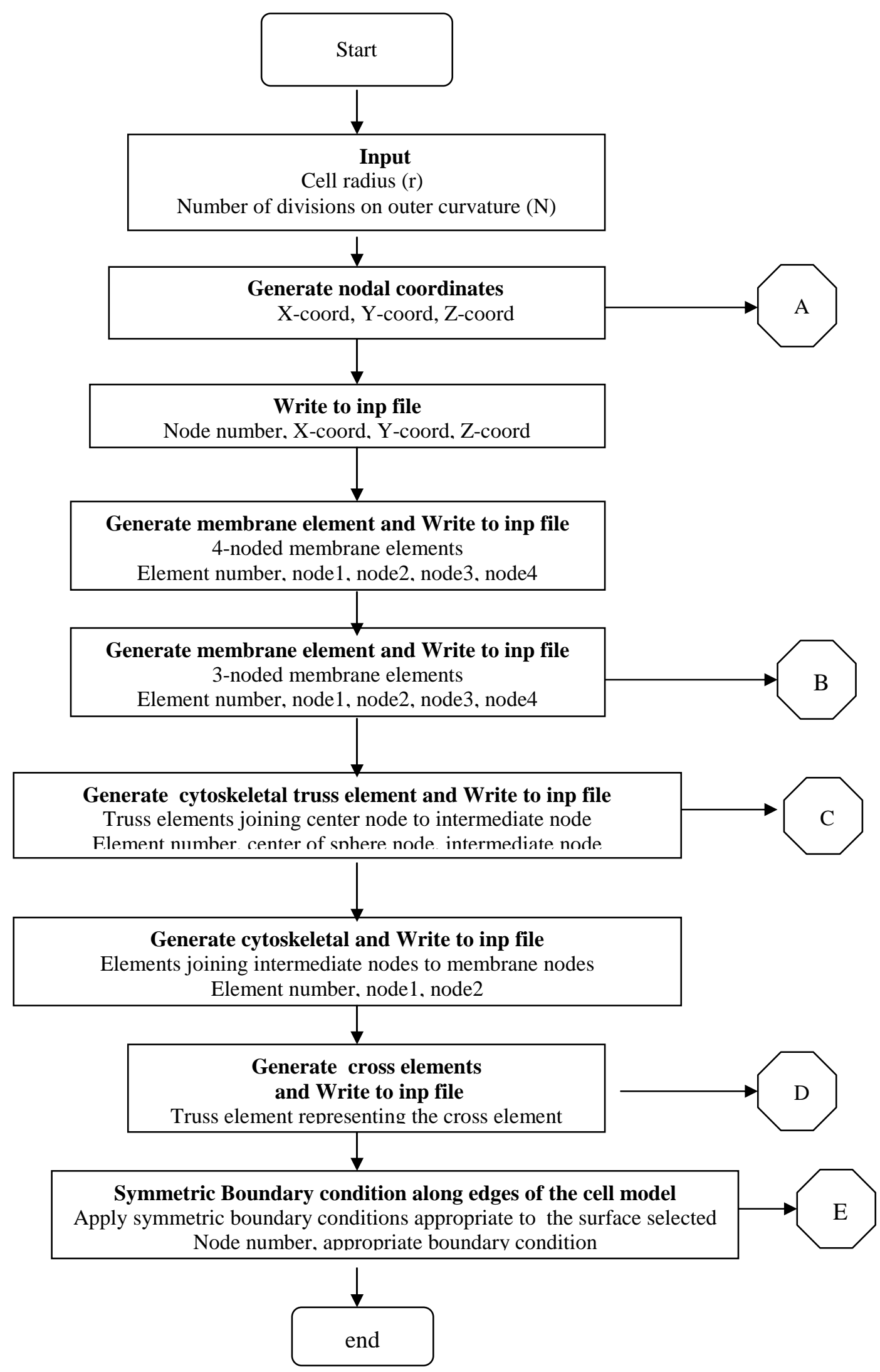



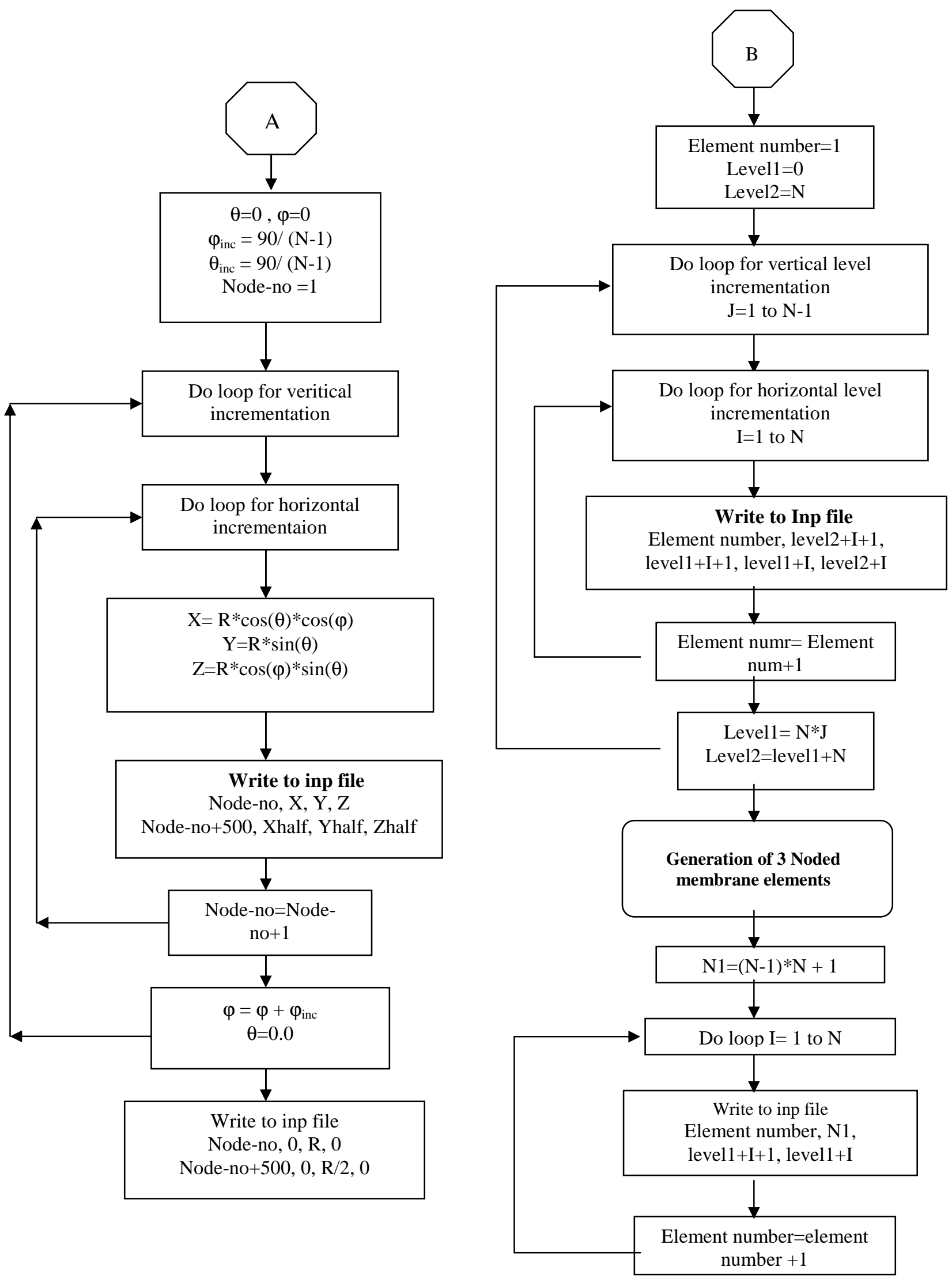


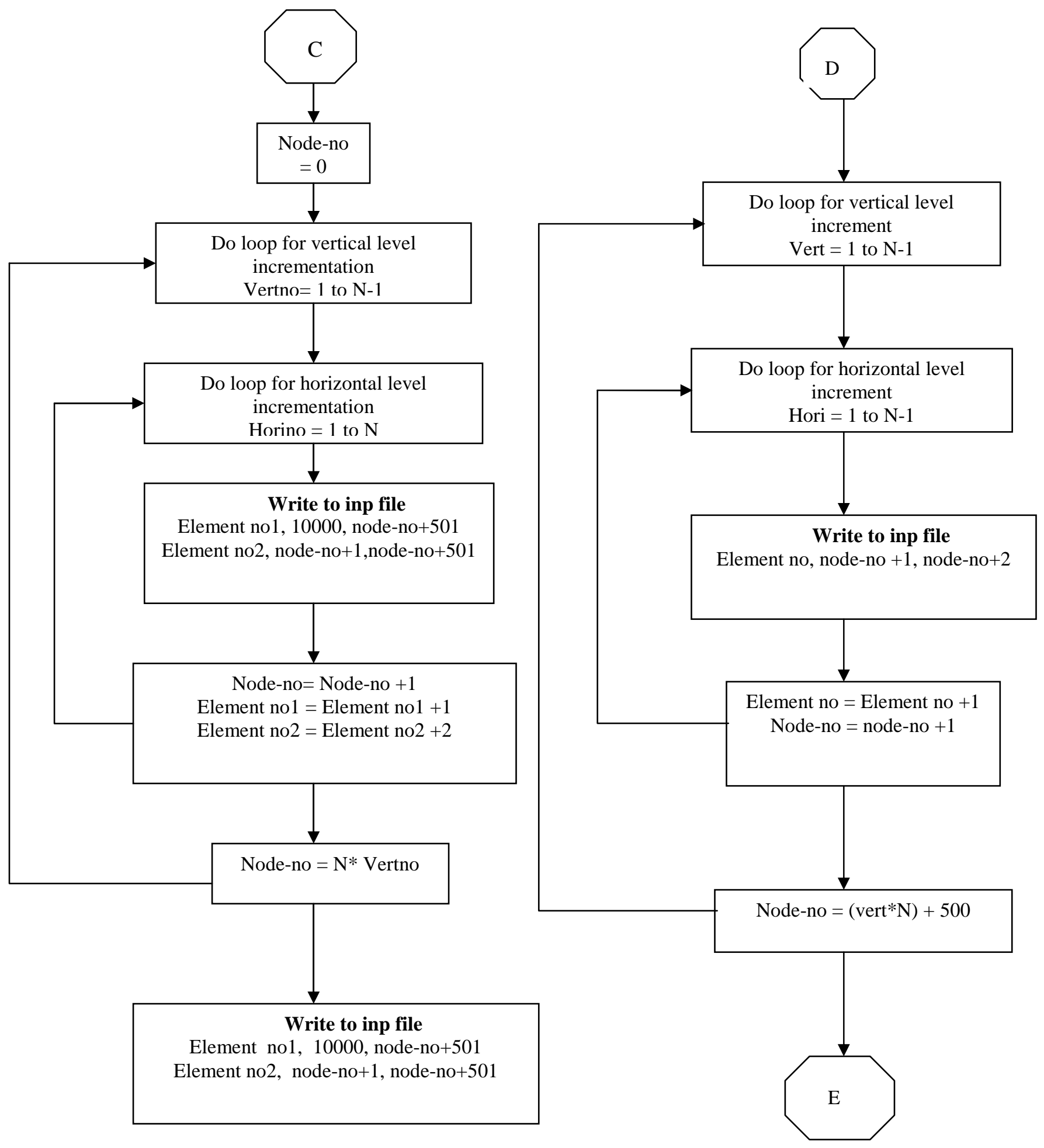




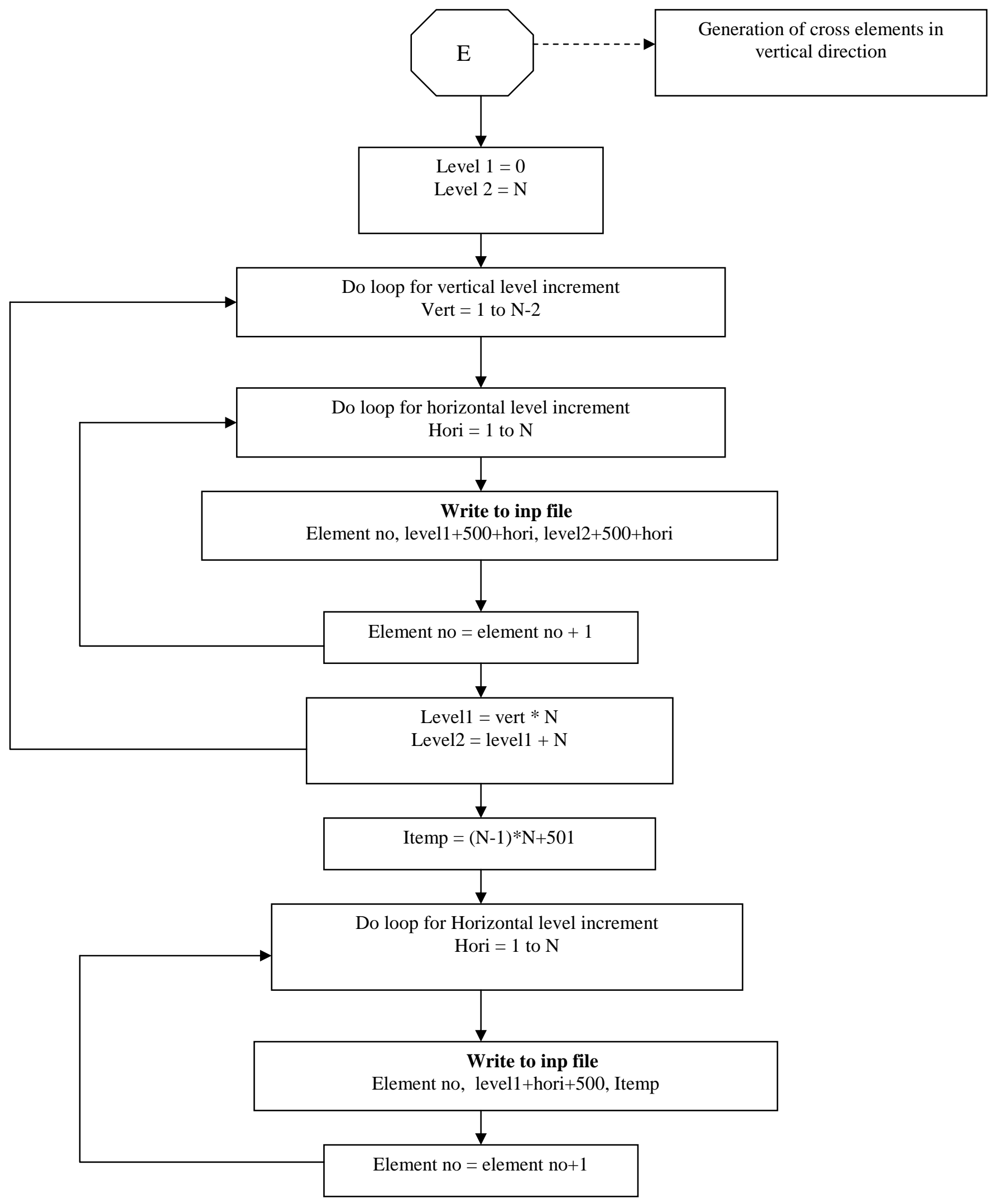


Right edge- constrained in 3-direction

Left edge - constrained in 1-direction

Bottom edge - constrained in 2- direction

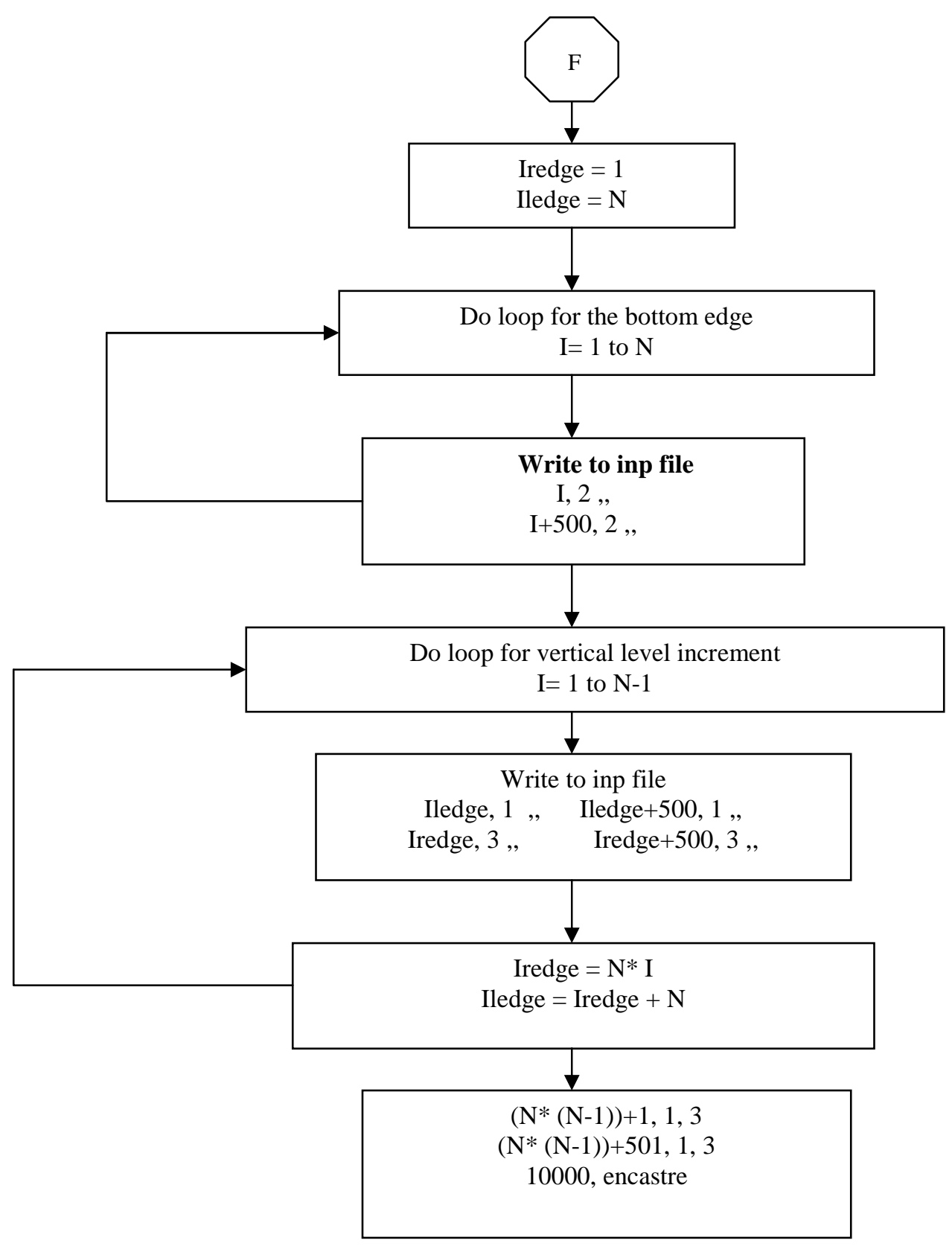


Symmetric boundary condition is applied to the nodes at the 3 edge- surfaces since only $1 / 8^{\text {th }}$ of the sphere is modeled. The nodes at bottom surface are constrained from translation in 2-Direction, the nodes at the left edge is constrained from translation in 1Direction and the nodes at the right surface are constrained in 3-Direction. The center of the sphere is constrained in all the directions. The steps involved in applying the boundary conditions are described in flowchart-F. This model is subjected to various kinds of loading conditions representing internal fluid pressure, cytoskeletal Pre-stress, External compressive pressure, etc., depending on the type of behavior to be studied with the model. The various steps involved in creating the 3D model of the cell is shown in the flowchart. Different Analysis is performed to study the behavior of this cell model to various conditions.

\subsection{Bulk modulus extraction program}

After the analysis is run, ABAQUS software stores results (deformation, stresses, strains etc.,) in a separate file with a name "filename" fil the filename is same as that used in the input file. A user subroutine is written to extract the original and deformed coordinates and the membrane elements and there connected nodes from the .fil file. The steps involved in extraction of nodal results are shown in flowchart. The original and deformed coordinates are extracted for the nodes related to membrane elements. The steps involved in extracting the nodal results related to membrane elements are shown in flowchart B, C and D. The overall volume is calculated by considering each membrane element as a pyramid with its apex as the center of the sphere and membrane element nodes as its base as shown in fig 9 . The original and deformed volume of the pyramid is calculated from the results extracted results from .fil file for the nodes connected to the membrane elements. The steps involved in 
calculating the volume are shown in flowchart E. The volume of the pyramid is calculated similarly for all the membrane elements (4-Noded and 3-Noded) and summed to get the original and deformed volume of the sphere. Change is volume $(\Delta \mathrm{V})$ is calculated from the original volume (V) and deformed volume. Equivalent Bulk modulus of the fluid filled model is calculated from the following formula.

$$
K=\frac{\text { Applied }- \text { Pr essure }}{\Delta V / V}
$$

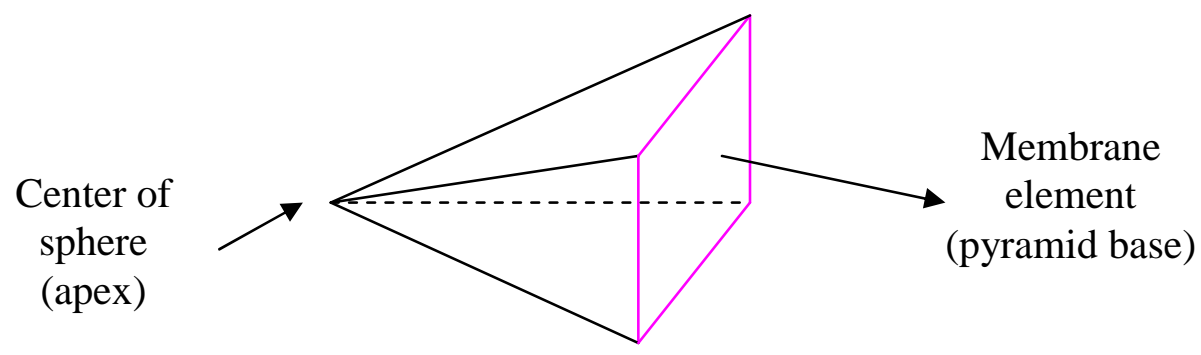

Figure 9 Pyramid structure

Pyramid with its apex as center of the cell and the base as the membrane element. Volume is calculated for all the membrane elements and added to get the overall cell volume. The formula for volume of the pyramid $=($ Area of base $*$ height $) / 3$ 


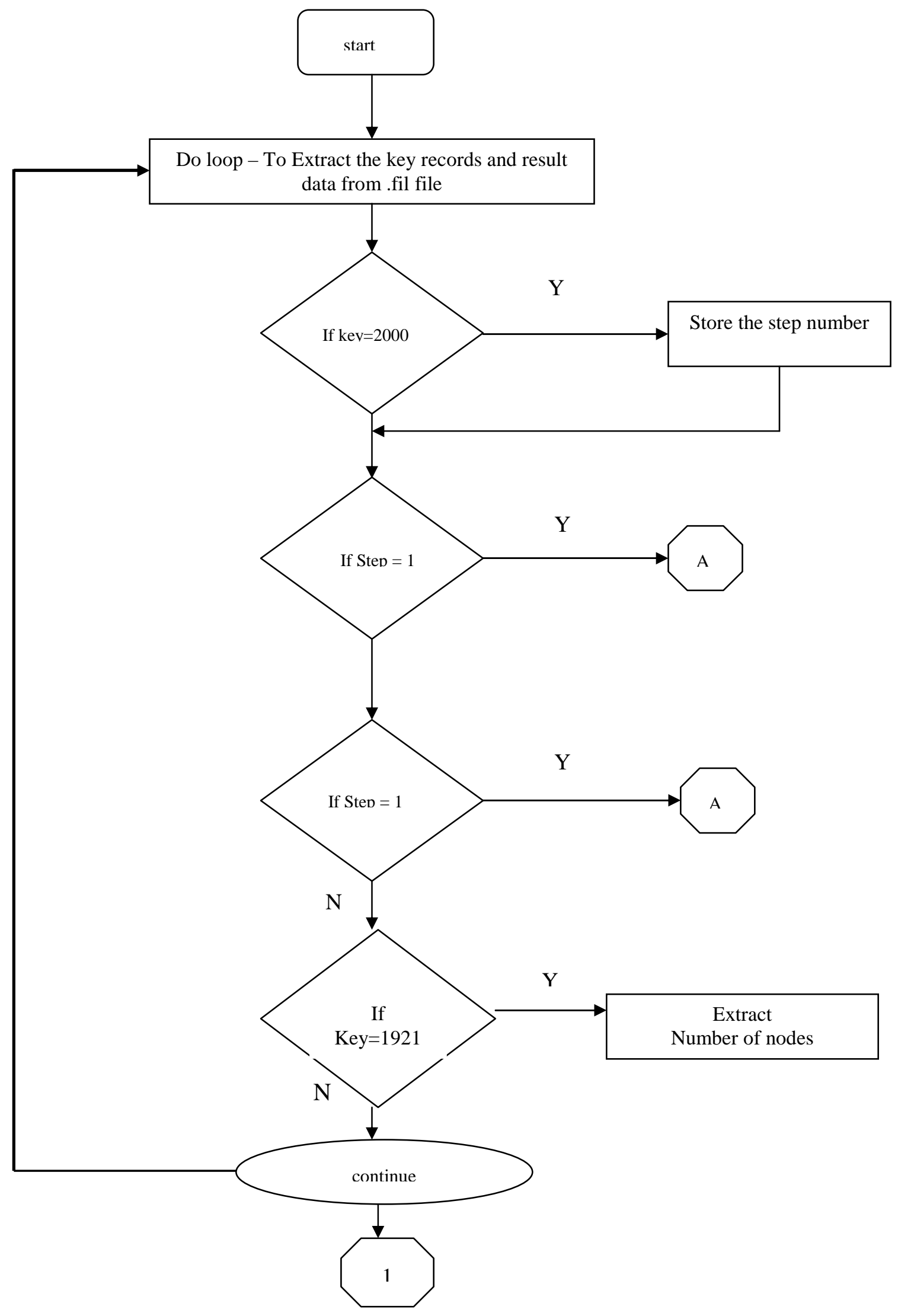




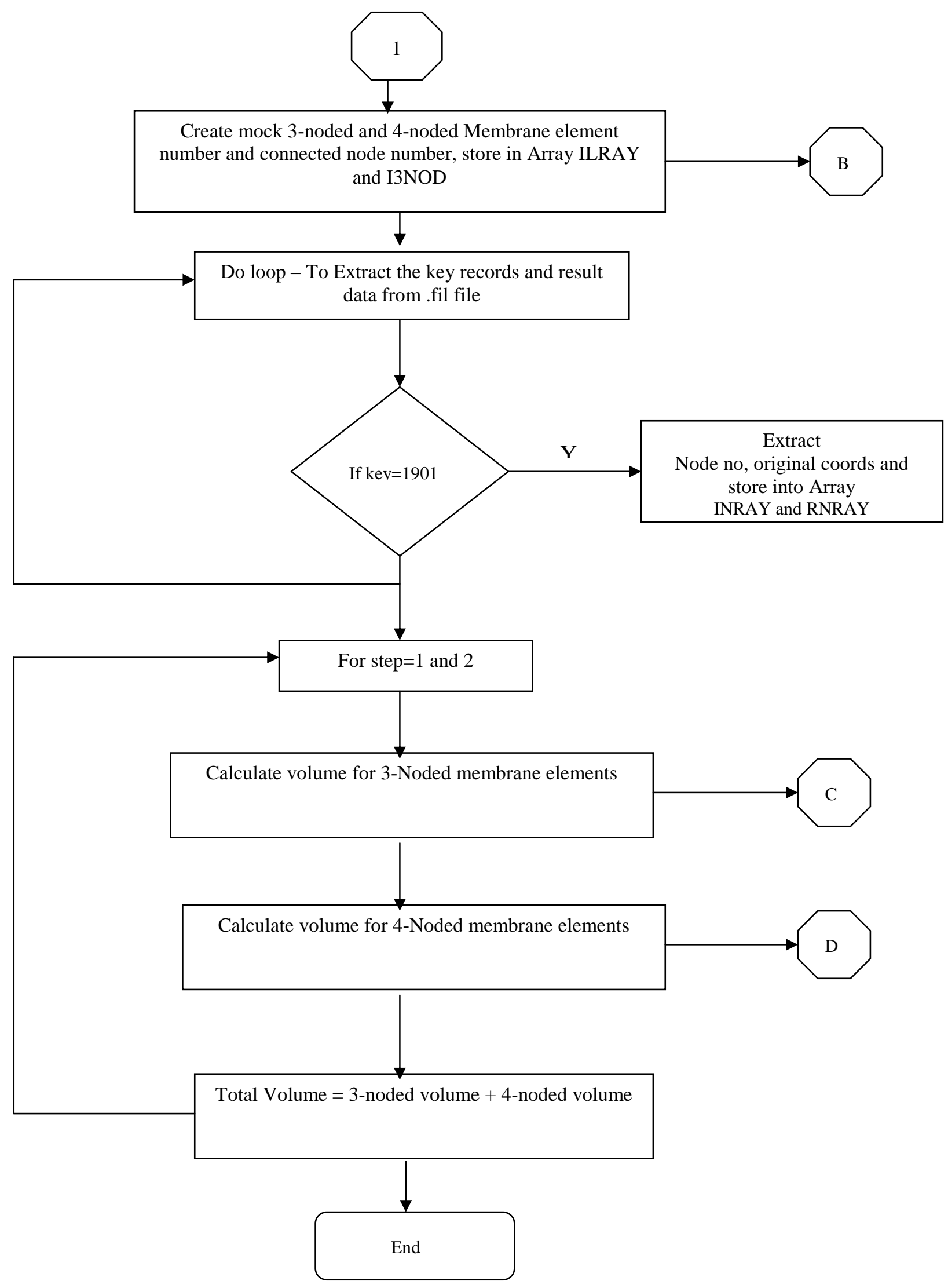




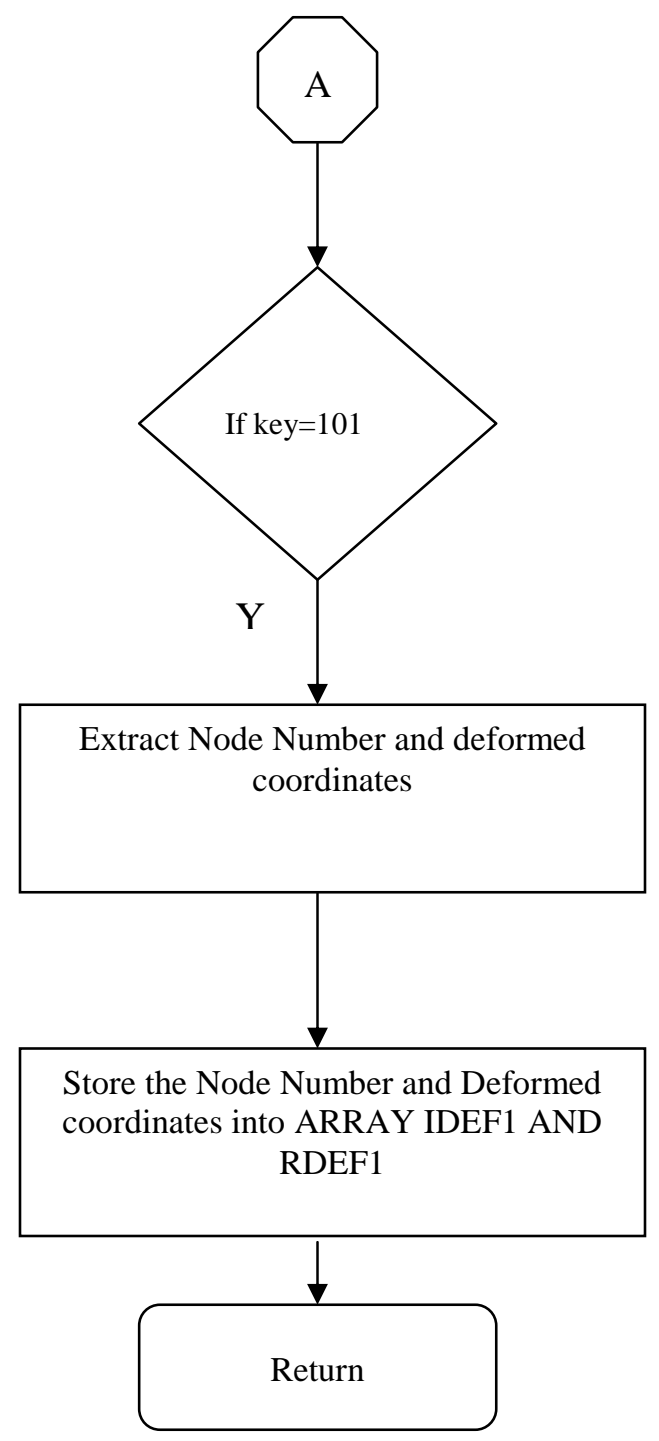




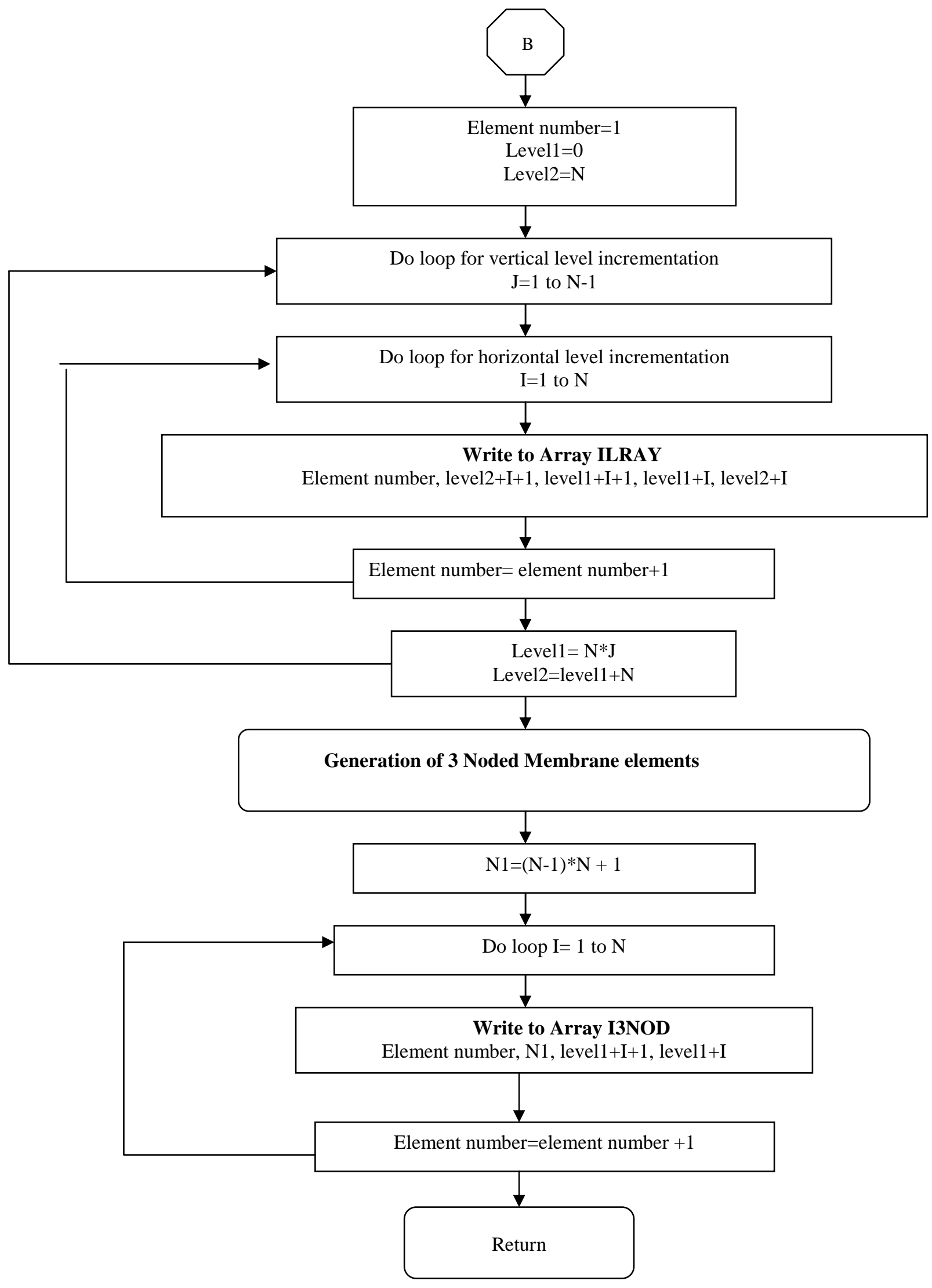




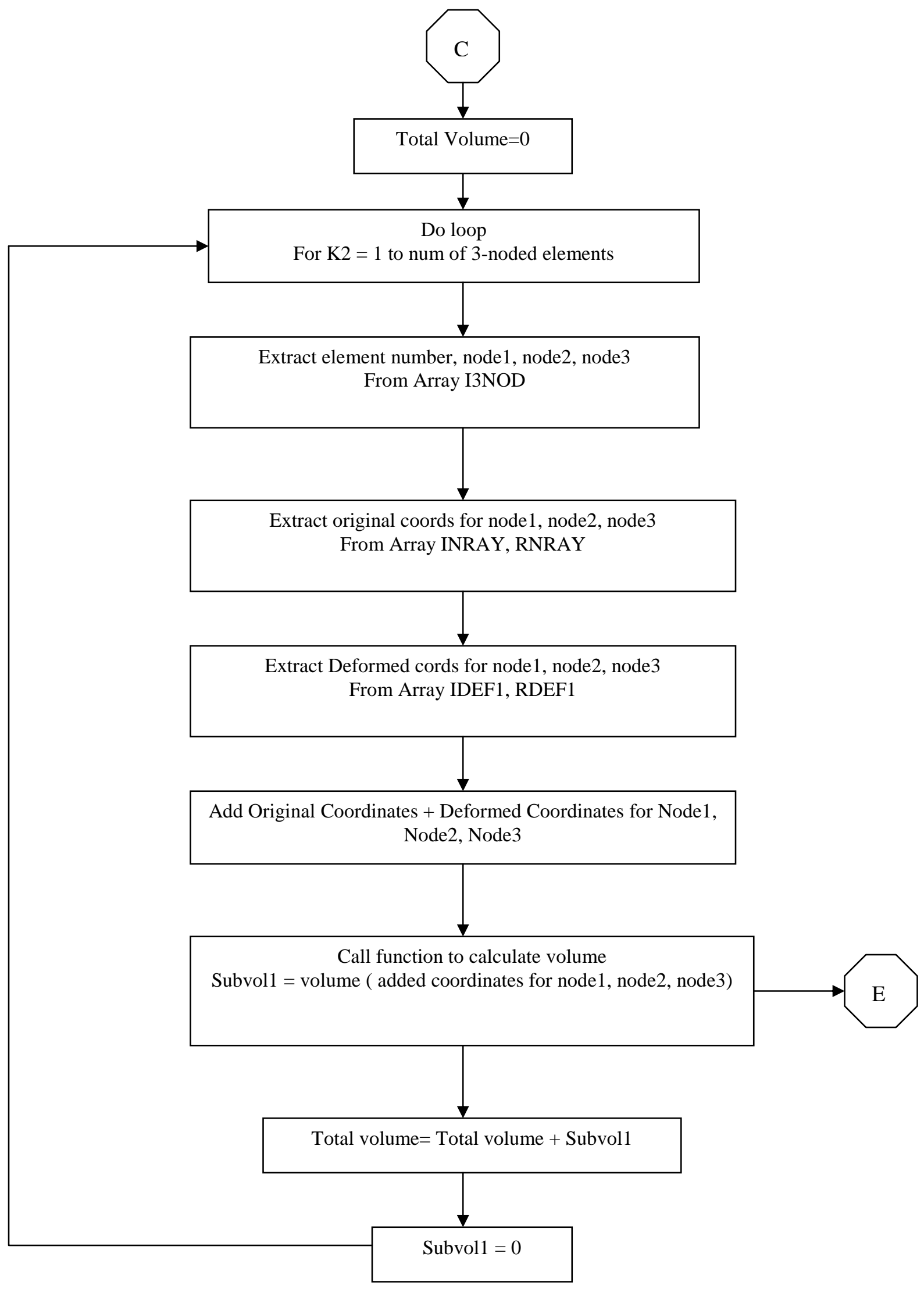




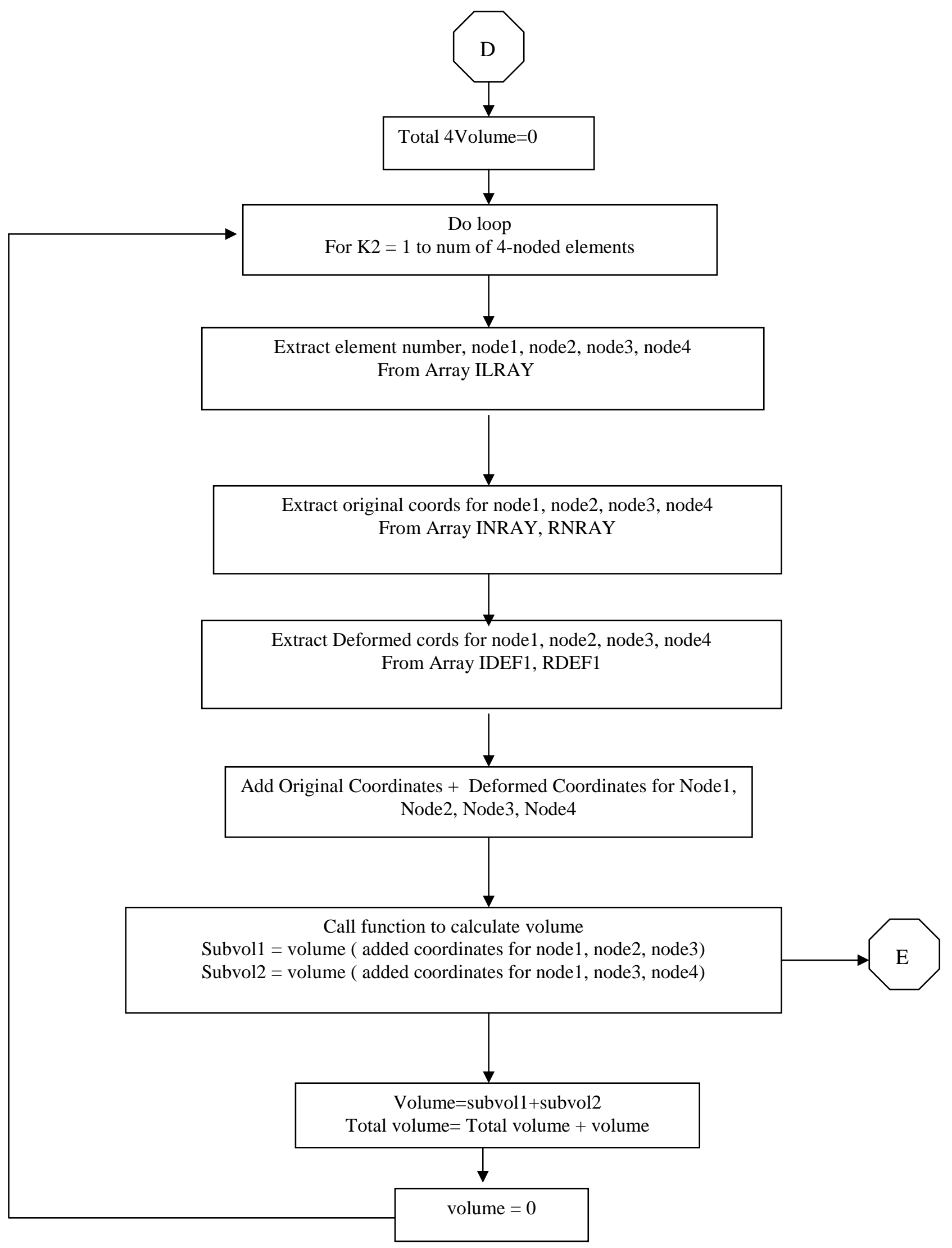




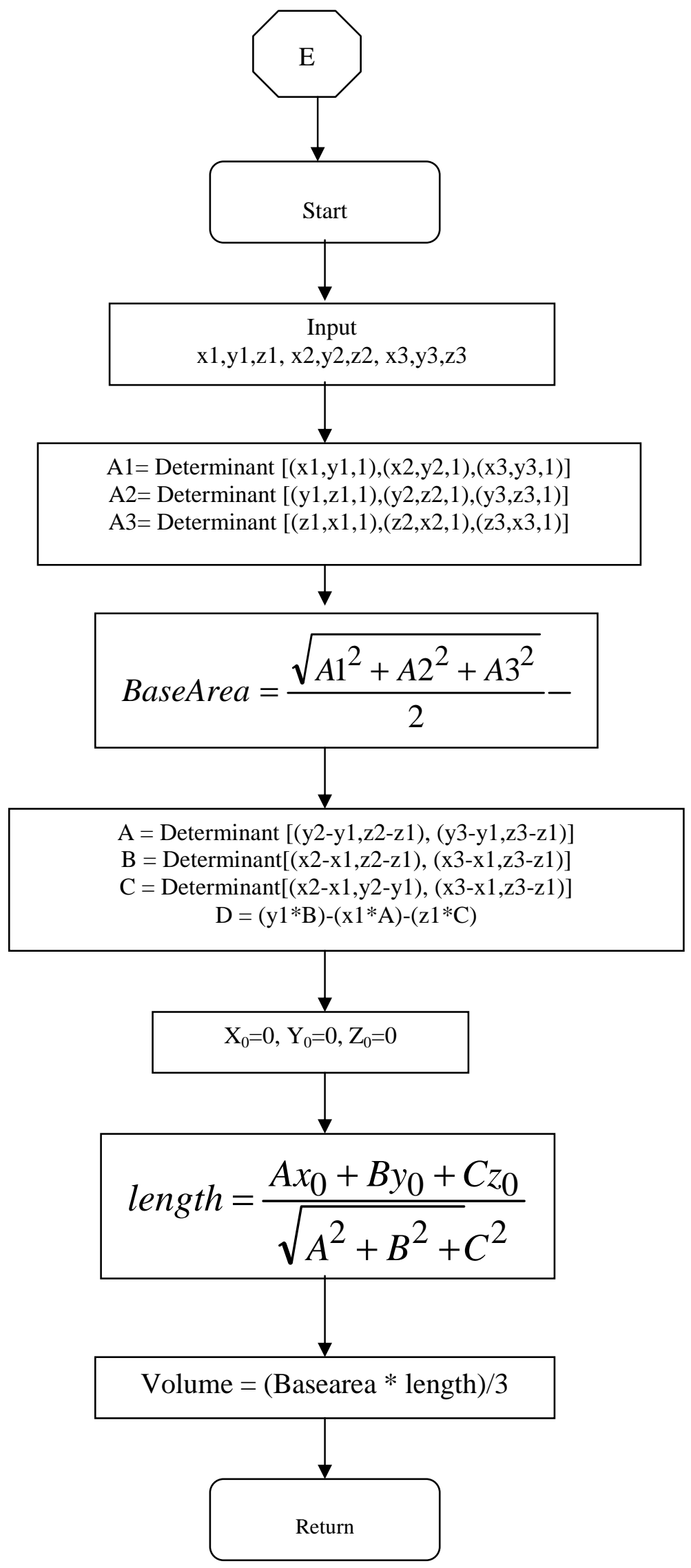




\subsection{Validation}

\section{Fluid filled model Vs continuum model}

The Equivalent bulk modulus is calculated from the extracted deformation data of the 3D cell Finite Element Model. Young's Modulus (E) is calculated from the Equivalent Bulk Modulus (K) from the following formula.

$$
K=\frac{E}{3(1-2 v)}
$$

A $1 / 8^{\text {th }}$ Continuum spherical FE model is created with its radius same as that of the 3D-cytoekeleta model. The young's modulus obtained from the CSK model is input as material property to the $3 \mathrm{D}$ continuum model. The continuum model is subjected to same external loading as that applied to the 3D CSK model. The deformation as a measure of change in radius $(\Delta \mathrm{R})$ is compared for both the CSK model and continuum model. The procedure for this validation is shown in fig 10. This validation is performed to gain confidence on the bulk modulus calculation program written for the CSK model as well as the deformation results obtained from the CSK model. 

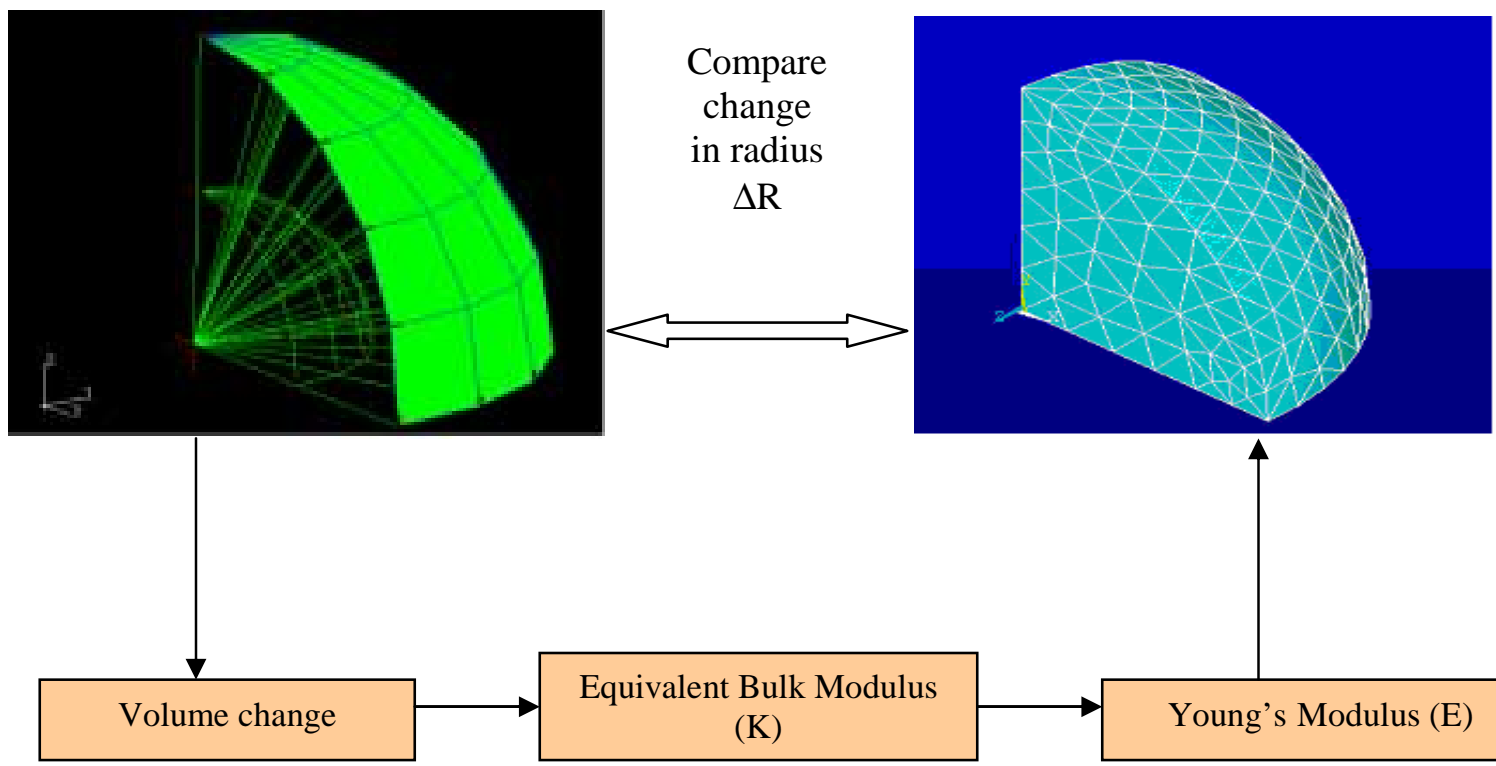

Equivalent Bulk Modulus (K)

Young's Modulus (E)

Figure 10 Procedure for cytoskeletal cell model validation.

Bulk modulus extracted from the cytoekeletal model is input as material property to continuum model. The deformation for both the model is compared

\begin{tabular}{|c|c|c|}
\hline \multirow{2}{*}{$\begin{array}{c}\text { Number of cytoskeletal } \\
\text { elements }\end{array}$} & Cytoskeletal model & Continuum model \\
\cline { 2 - 3 } & 0.485 & 0.473 \\
\hline 180 & 0.276 & 0.268 \\
\hline 420 & & 0.2005 \\
\hline 612 & 0.2053 & \\
\hline
\end{tabular}

Table 1 Accuracy of the validation of cytoskeletal model with a closed form solution.

The table shows the accuracy of the deformation measured as change in radius of the sphere. Also as the number of cytoskeletal elements increases the stiffness of the cell increases, as can be seen that the change in radius decreases. 
Following the procedure described in Fig-10, the deformations for a given number of cytoskeletal elements in a cell model was compared with that of a continuum model. The results are shown in table-1. Table-1 shows the deformation (measured in terms of change in radius $\Delta \mathrm{R}$ ) for the cytoskeletal model matched closesly with the continuum model for 3 different cases. Also the results show that as the number of cytoskeletal elements increase the overall deformation of the cell subjected to compressive loading decreased.

\section{Continuum model Vs Closed form Solution}

The accuracy of the results obtained from the continuum FE model is compared with a closed form solution of Spherical Inclusion inside a Medium. The elasticity solution for the model shown in Fig-10 was solved by Goodier [39]. The model was considered axissymmetric about $\mathrm{X}$ and $\mathrm{Y}$-axis. The spherical inclusion has material properties of $\mathrm{E} 1, \mathrm{v} 1$ and the medium with properties E2, v2. A uniform pressure $\mathrm{T}(\mathrm{N} / \mathrm{mm})$ was applied at the infinite distance from the inclusion. The deformation and stresses in radial and tangential direction at any location in the medium is given in the equations described below.

$$
\begin{gathered}
U_{r}^{b}=-\frac{A}{r^{2}}-\frac{3 B}{r^{4}}+\left(\frac{5-4 v_{b}}{1-2 v_{b}}\right) \frac{2 C}{3 r^{3}}+\left[\frac{-9 B}{r^{4}}+\left(\frac{5-4 v_{b}}{1-2 v_{b}}\right) \frac{C}{r^{2}}\right] \cos 2 \theta+\frac{T r}{2 E}\left[\left(1-v_{b}\right)+\left(1+v_{b}\right) \cos 2 \theta\right] \\
U_{\theta}^{b}=-\left[\frac{6 B}{r^{4}}+\frac{2 C}{r^{2}}\right] \sin (2 \theta)-\frac{T r}{2 E}\left(1+v_{b}\right) \sin 2 \theta
\end{gathered}
$$




$$
\begin{gathered}
\sigma_{r r}^{b}=2 \mu_{b}\left\{\frac{2 A}{r^{3}}+\frac{12 B}{r^{5}}-\frac{2 v_{b}}{\left(1-2 v_{b}\right) r^{3}}+\left[-\frac{2\left(5-v_{b}\right) C}{\left(1-2 v_{b}\right) r^{3}}+36 \frac{B}{r^{5}}\right] \cos 2 \theta\right\}+T \cos 2 \theta+\frac{T}{2} \\
\sigma_{r \theta}^{b}=2 \mu_{b}\left[\frac{25 B}{r^{5}}-\left(\frac{1+v_{b}}{1-2 v_{b}}\right) \frac{2 C}{r^{3}}\right] \sin (2 \theta)-\frac{T}{2} \sin (2 \theta)
\end{gathered}
$$

A,B,C - functions representing the mechanical properties of inclusion and medium. Shown in Appendix-9. Goodier, 1933

There are separate sets of formulas for calculated the stress and deformation in radial and tangential direction inside the inclusion. Both the set of formulas should give same deformation at the boundary of the inclusion and medium. The boundary condition is symmetric about $\mathrm{Y}$-axis at left edge and $\mathrm{X}$-axis at the left edge. Also the boundary condition between medium and inclusion is no slip condition.
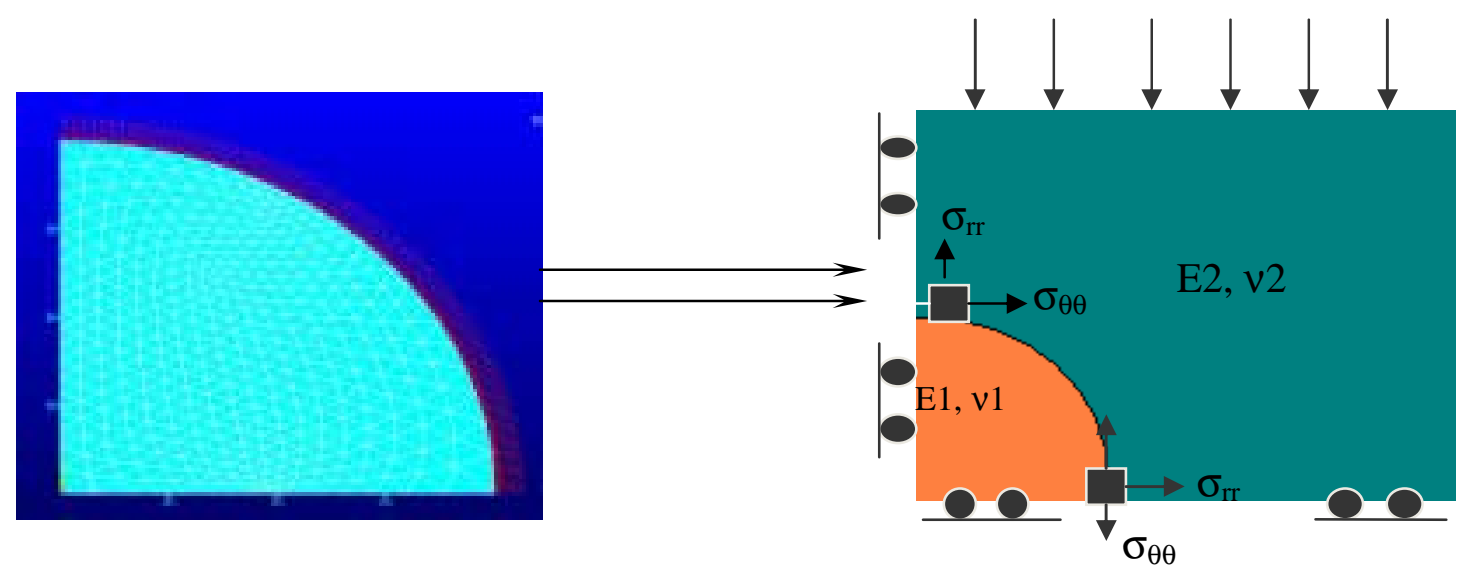

Continuum model

Spherical inclusion inside a medium Goodier, 1933

Figure 11 Validation of Finite element model with closed form solution Comparision of the continuum finite element sphere model with a closed form solution of spherical inclusion inside a medium. 

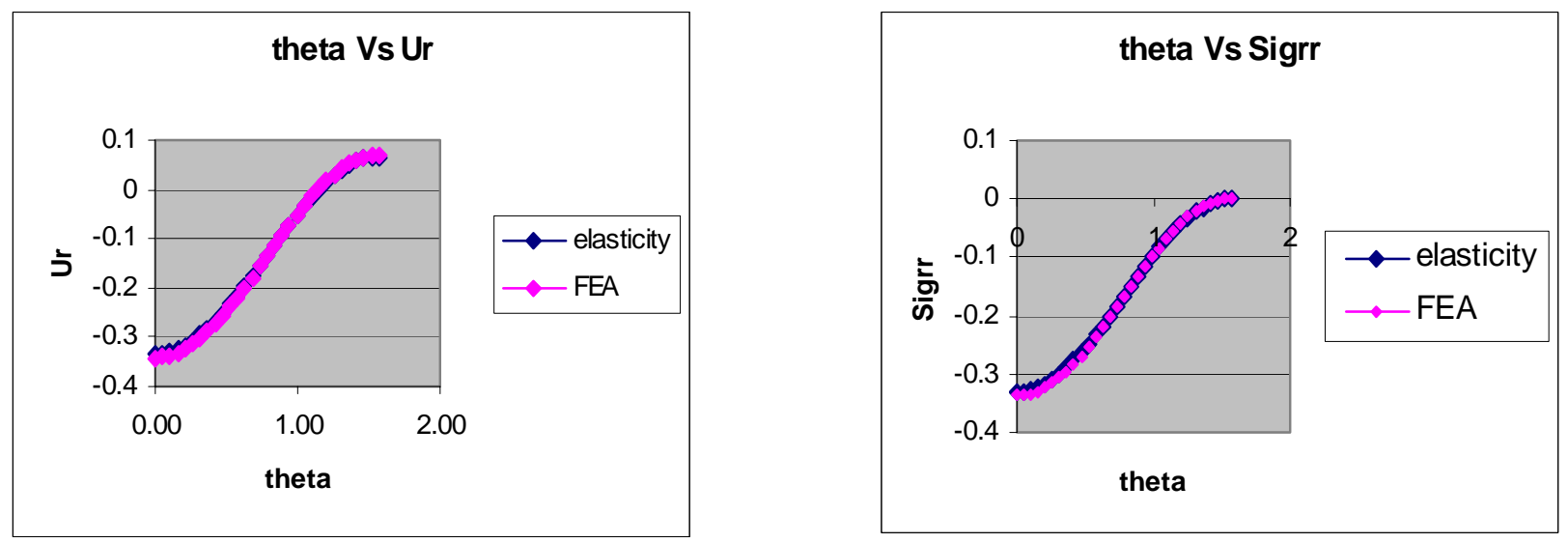

Figure 12 Accuracy of continuum FE spherical model with a closed form solution.

The graph shows deformation in radial direction (Ur) for the Continuum FE model, which goes close to that of closed form solution at the boundary between the medium and the inclusion.

Figure 13 Accuracy of continuum FE spherical model with a closed form solution.

The graph shows stress in radial direction $\left(\sigma_{\mathrm{r}}\right)$ for the Continuum FE model, which goes close to that of cloesd form solution at the boundary between the medium and the inclusion.

A program was written using MAPLE to calculate the deformation and stress at the boundary between the medium and the inclusion. The MAPLE code is given in Appendix 4. The results from the closed form solution were compared with that of the Continuum FE model as shown in Fig-12 and Fig-13. The deformation in the radial direction and the stress in radial direction at the boundary between the inclusion and the medium at various angles of theta is plotted for both the FE continuum model and the Goodier's model [39]. The accuracy of the results between the closed form solution and the continuum FE model is shown in Fig12 and Fig-13. The closeness of the FEA results with that of the closed-form solution gives us the confidence in believing results of the other finite elements models for which calculating a closed form solution is not feasible. 


\subsection{Result}

Having validated the model with a closed form solution, we take a look at the influence of different parameters on the bulk modulus of the cell. First the effect of the Young's modulus of the cytoskeletal elements on the bulk modulus $(\mathrm{K})$ of the cell was studied. Fig-14 shows that 10,000-fold increase in Young's modulus of the cytoskeletons increased the buck modulus by 5 fold. Next the effect of number of cytoskeletal elements on the bulk modulus of the cell was studied. Fig-15 shows that a 1500 fold increase in number of cytoskeletal elements caused only a 2 -fold increase in the bulk modulus of a cell. Next when 4 fold increased the internal fluid pressure, it caused the bulk modulus to increase by 2 fold. This showed that the internal fluid pressure is more important parameter compared to the cytoskeletal elements, when it comes to the overall stiffness of the cell.
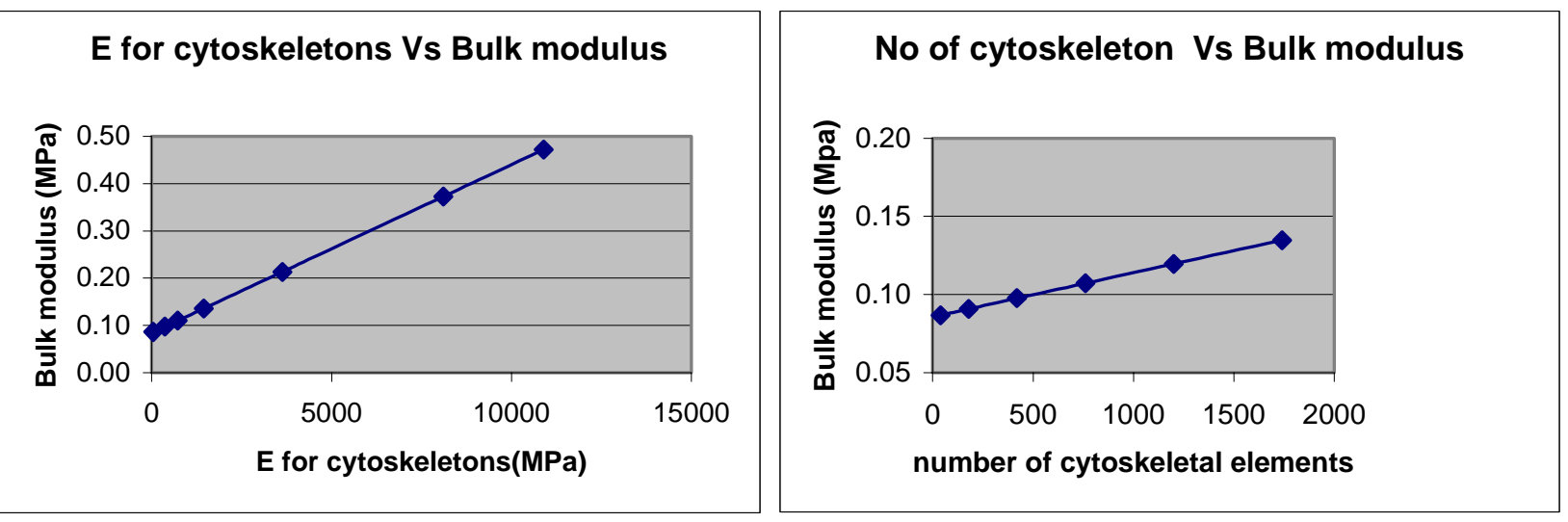

Figure 14 Effect of Young's modulus of cytoskeletons on the bulk modulus of cell.

A 10,000 fold increase in Young's modulus of cytoskeletal elements caused only a 5 fold increase in the bulk modulus of the cell

Figure 15 Effect of number of cytoskeletal elements on the bulk modulus of cell.

A 1500 fold increase in \# of cytoskeletal elements caused only a 2 fold increase in the bulk modulus of the cell. 


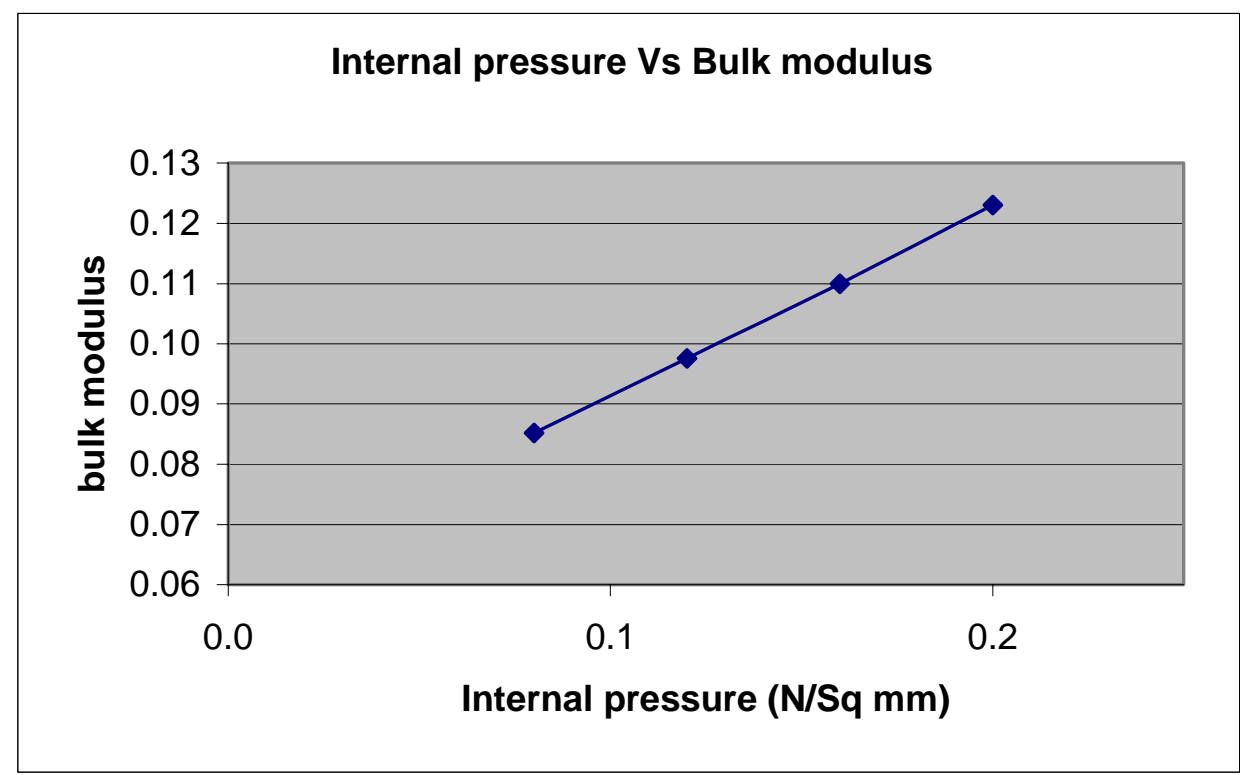

Figure 16 Effect of internal fluid pressure on the bulk modulus of the cell.

A 4 fold increase in the internal fluid pressure caused a 2 fold increase in the overall bulk modulus of the cell.

\subsection{Discussion}

The model was first validated. Then the effect of various structural parameters on the overall stiffness of the cell was studied. The parametric study shows that internal fluid pressure has more effect on the overall stiffness of the cell compared to the number of cytoskeletal elements and its material property. The reason behind such a behavior could be due to the fact that volume fraction of the cytoskeletal elements are very less compared to that of the whole cell. Therefore if we model a cell with a very large number of cytoskeletal elements then it could lead to having a more effect on the overall stiffness of the cell. Also by varying all these 3 structural parameters, we can make the cell to be 100 times stiffer. This could explain the various behaviors related to chondrocytes being stiffer than other cells. 


\section{Chapter 4: Buckling Behavior of the microtubule}

\subsection{Introduction}

Microtubules are known to be one of the structural elements of a cell that contribute towards cell stiffness [32] [40]. Microtubules are known to be one of the major compression bearing elements in a cell $[32,33]$. Experiments show that when the microtubule elements were dissolved using drugs, the cell expanded, which supports the fact that the microtubules are in compression [33] [30].

Experiments also show that not only does the microtubule take up compression but also they are prone to buckling [30]. Fig-17 shows the buckling of microtubule when it polymerizes and expands impringing the outer stiff cortex [30]. The arrow in the figure shows the buckled microtubule. Various mechanical models like ingber's Tensegrity model considers cell as pre-stressed actin-microtubule filaments interconnected such that they stabilize to resist external loads[41]. The slenderness ratio (Length/radius $=3000 / 12$ ) of microtubules is 250 . this high slenderness ratio makes them prone to buckling.

Figure 17 Bucking of Microtubules

Bucking of microtubule elements as it polymerizes and hits the stiff cortex of cell. The arrow mark shows microtubule buckling. Figure from Wang et al., [30]

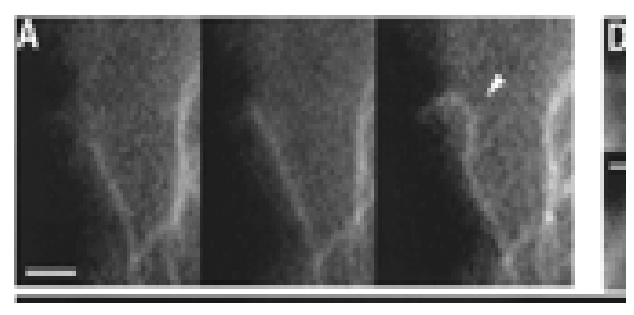

Volokh et al., [42] and Coughlin et al., [43] studied the buckling behavior of the Ingber's tensegrity model and showed the stiffening response of the buckled microtubule in a 
tensegrity structure. So buckling behavior of the microtubule for our 3D cell model also studied. ABAQUS, a finite element software was used for this purpose.

\subsection{Buckling - a review}

When a long thin column is subjected to axial compressive load (P) it compresses axially. As the applied load $\mathrm{P}$ is increased till it reaches a critical load $\mathrm{P}_{\mathrm{cr}}$ the column shows a compressive behavior. But for a load $\mathrm{P}>\mathrm{P}_{\mathrm{cr}}$ the column will start to buckle and will settle in a new equilibrium position, with considerable lateral deformation. The simplified model is shown in Fig-18, which has a pinned- boundary condition subjected to compressive loading. Euler's formula for finding the critical load for a column with pinned end boundary conditions is

$$
\mathrm{P}_{\mathrm{cr}}=\pi^{2} \mathrm{EI} / \mathrm{L}_{\mathrm{e}}^{2}[44]
$$

E - Young's modulus

I - Moment of Inertia $\left(=\pi R^{4} / 4\right.$ for circular $\left.C / S\right)$

$\mathrm{L}_{\mathrm{e}}-$ Effective length (varies with different Boundary conditions)

The $\mathrm{L}_{\mathrm{e}}$ for different boundary condition is given in Table-1

\begin{tabular}{|c|c|}
\hline Pin-pin & $\mathrm{L}_{\mathrm{e}}=\mathrm{L}$ \\
\hline Fixed-free & $\mathrm{L}_{\mathrm{e}}=2 \mathrm{~L}$ \\
\hline Fixed - pinned & $\mathrm{L}_{\mathrm{e}}=0.7 \mathrm{~L}$ \\
\hline Fixed-Fixed & $\mathrm{L}_{\mathrm{e}}=0.5 \mathrm{~L}$ \\
\hline
\end{tabular}

Table 2 Effective length for a column with different end conditions.

The effective length is actual length for pin-ended condition. The effective length half the actual length for fixed free condition. The effective length is twice actual length for fixedfixed condition 
A microtubule is considered as a single column and the buckling behavior is studied.

The microtubule properties were taken from the literature

$\begin{array}{ll}\text { Outer Diameter } & -25 \mathrm{~nm}[21] \\ \text { Thickness } & -2.7 \mathrm{~nm} \\ \text { Young's modulus } & -1.2 \mathrm{E}+03 \mathrm{Mpa}[22] \\ \text { Length } & -3 \mu \mathrm{m}\end{array}$

\section{Units :}

Load $-\mu \mathrm{N}=10^{-6}$ pico Newton

Length - $\mu \mathrm{m}$

Stress $-\mu \mathrm{N} / \mu \mathrm{m}^{2}=\mathrm{MPa}$

Figure 18 columns with pin-ended boundary condition.

The top surface is subjected to axial compressive loading. The bottom node is pinned to the ground.

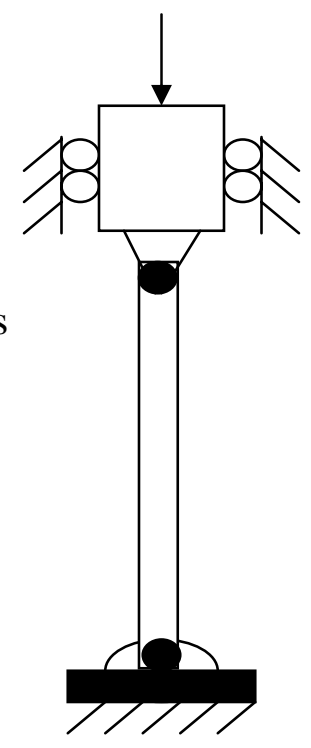


Theoretical calulation of $\mathrm{P}_{\mathrm{cr}}$ for isolated Microtubule with pin-ended boundary condition are: $L e=3 \mu m[43](\mathrm{Le}=\mathrm{L}$ for a pin-pin boundary condition $)$

$R=0.01258 \mu \mathrm{m}$

$E=1.2 * 10^{3} \mu \mathrm{N} / \mu \mathrm{m}^{2}$

$I=\pi R^{4} / 4=1.791 * 10^{-8} \mu m^{4}$

$P_{c r}=23.57 * 10^{-6} \mu N$

$P_{c r}=$ 23.57 Pico Newton

ABAQUS Finite element software is used to perform the buckling analysis for complex structure like cell model. Two types of buckling analysis can be performed in ABAQUS one is Eigen value buckling analysis, which is used to extract the $P_{\text {cr }}$ for a structure while the other is RIKS analysis, which is used to study the post buckling behavior of a structure.

\subsection{Eigen Value Bucking Analysis}

The Critical load for buckling $\mathrm{P}_{\mathrm{cr}}$ is obtained using Eigen Value Analysis in ABAQUS. The microtubule column was modeled with 2-noded beam elements (element type B31). Fig-18 shows the model of a single column with microtubule properties as described above. 
The Eigen value-Bucking analysis is used to extract the $\mathrm{P}_{\mathrm{cr}}$ for the model. The ABAQUS commands for performing the Eigen Value Buckling is:

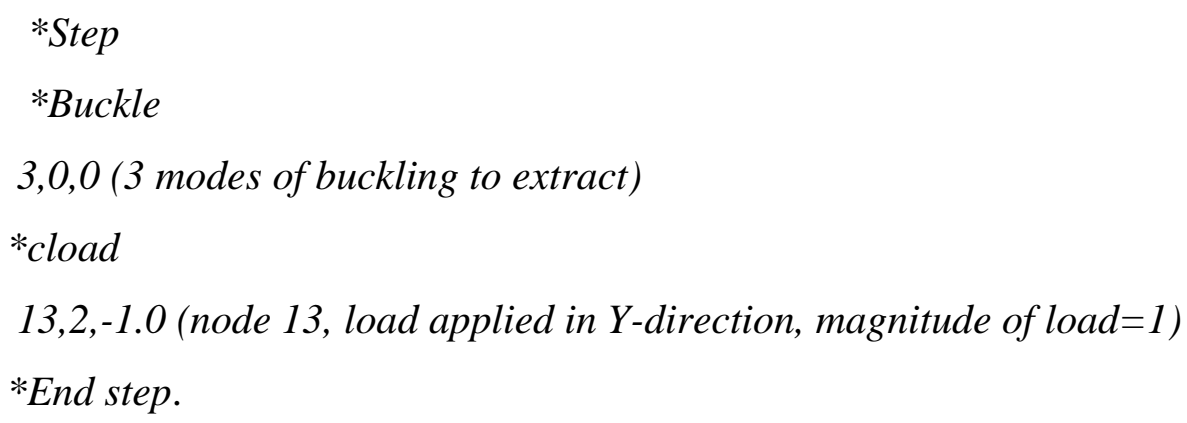

The $\mathrm{P}_{\text {cr }}$ from ABAQUS matched well with the theoretical value of $23.57 \mathrm{PN}$. The Eigen value analysis was verified with the theoretical calculations for different boundary conditions, which is shown in table 3

\begin{tabular}{|c|c|c|}
\hline Boundary condition & $\mathbf{P}_{\mathrm{cr}}-$ ABAQUS & $\mathbf{P}_{\mathrm{cr}}-$ Theory \\
\hline Pin-roller & 23.68 & 23.57 \\
\hline Fixed-free & 6.48 & 6.47 \\
\hline Fixed-pin & 53.7 & 52.81 \\
\hline
\end{tabular}

Table 3 Comparison of ABAQUS Eigen value results with theoretical calculation.

The closeness of the results obtained from ABAQUS with that of theoretical values for microtubule column.

\section{Eigen Value analysis of Microtubule column 2-Step loading}

The behavior of microtubule column is studied for a multiple step loading.

Step1-apply a small vertical loading to compress the vertical column.

Step2- apply bucking load.

Theoretical value of the Pcr is found to be around 24 PN (for 0 preload). The following table gives the Pcr for the column with different dead load (step-1). 


\begin{tabular}{|c|c|c|}
\hline $\begin{array}{c}\text { Step-1 load (dead load) } \\
\mathrm{pN}\end{array}$ & $\begin{array}{c}\text { Pcr (ABAQUS) } \\
\mathrm{pN}\end{array}$ & $\begin{array}{c}\text { Total Pcr } \\
\text { Dead load + Pcr (abaqus) }\end{array}$ \\
\hline 0 & 26 & 26 \\
\hline 1 & 25 & 26 \\
\hline 2 & 24 & 26 \\
\hline 10 & 16 & 26 \\
\hline 20 & 6 & 26.001 \\
\hline 26 & 0.001 & \\
\hline
\end{tabular}

Table 4 Effect of dead load on the critical buckling load.

As the dead load is increased from 0 PN to $26 \mathrm{PN}$ the eigen value extracted in ABAQUS decreases from the actual critical load of 26 PN to 0.001 PN. The total load of dead load + the eigen value extracted is equal to the actual critical load for different cases of dead load applied.

From the above analysis, the vertical load acts as a dead load and ABAQUS calculates the critical load (Pcr) for bucking as

$\operatorname{Pcr}($ Total $)=$ dead load + Applied load $* \lambda_{c r}$.

Thus a large dead load will reduce the applied load needed to buckle the column.

\subsection{Riks Instability Analysis}

Eigen value Buckling analysis is performed to obtain the critical buckling load only. The Eigen value analysis gives the normalized deformation values for the buckled column. Therefore to obtain the actual deformations for the buckled column, a riks procedure is performed. This is a 2-step analysis, in the first step - a small lateral load is applied to create 
an imperfection in the model, this load is called perturbation load. The second step is the vertical load applied causing the column to buckle. When the vertical load applied is greater than $\mathrm{P}_{\mathrm{cr}}$ the column starts to buckle and the behavior known as post bucking behavior is plotted as a relation between chord length Vs applied load. ABAQUS solves the unstable analysis in increment of the applied load called the load factor. The load factor usually ranges from 0 to 1 . For a particular increment, the deformation is calculated for a particular load factor. A user subroutine is written in FORTRAN to extract the nodal deformation and load factors for all the increments. This subroutine is shown in appendix 8. Chord length is the deformation of the top most node of the column in Y-Direction. The input file for riks analysis is shown in Appendix-6. The post-bucking plot of Chord length Vs Load is given in the Fig-19.

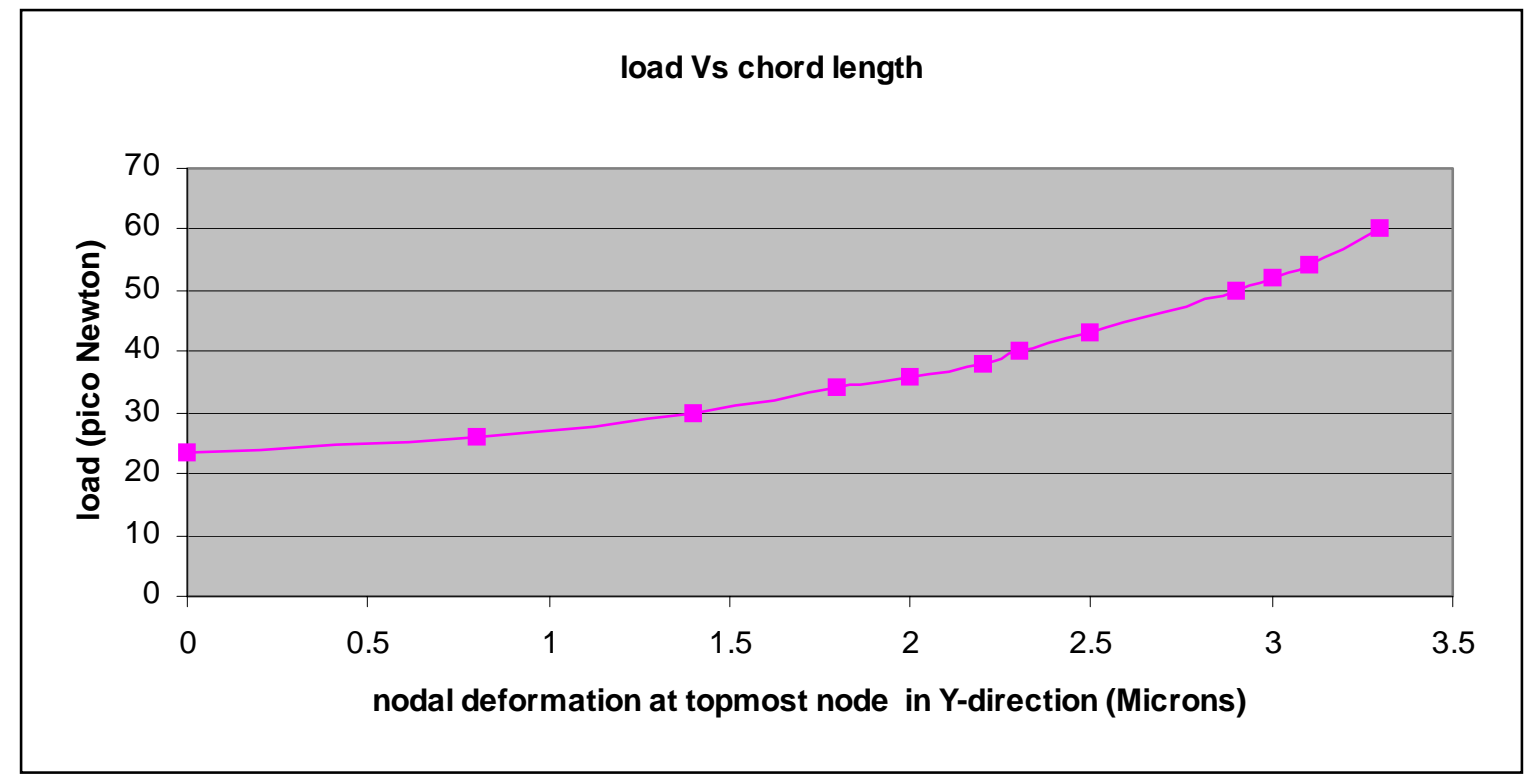

Figure 19 Post buckling of microtubule column

Post bucking analysis of Microtubule. The boundary condition used is Pinned-roller type. The column starts to buckle at 23.57 PN and further shows a post bucking behavior where even for a small increase in load there is a huge increase in deformation 
ABAQUS Commands for performing Riks analysis:

*Step

*static, riks

$0.01,1 .,, 1.0$

*cload

13,2,-23.57 (node 13 loaded in Y-direction, magnitude=23.57)

*End step.

A pipe Crossection is chosen as it resembles the Microtubule $C / S=190 \mathrm{~nm}^{2}$ [42] with thickness to be $2.7 \mathrm{~nm}$. Fig-19 shows the post buckling behavior of microtubule column. The plot shows the axial deformation of the top most node of the column Vs the applied load for a microtubule column. For a load below the critical load the deformation is very less. But once the critical load is reached the column starts to buckle and hence even for a slight increase in the load, there is a large deformation. This is shown in Fig-19.

\subsubsection{Validation}

\section{Riks Post-buckling of MT-Column with a closed-form solution}

The post bucking analysis Microtubule was performed by 2 different groups Couglin et al [43] and by Volokh et al [42]. Coughlin et al [43] predicted a post bucking behavior as shown in the fig 20, which shows the relation between chord length and applied load. Both Coughlin and Volokh et al., [42] predict the same $\mathrm{P}_{\text {cr }}$ but Volokh claimed Couglin et al [43] to be wrong. Volokh et al [42] predicted the post buckling behavior to be stiffer than that predicted by Coughlin for the same geometry conditions. Our post-buckling curve matches to that predicted by Volokh et al., [42]. This gives the confidence that the results predicted by ABAQUS are believable. 


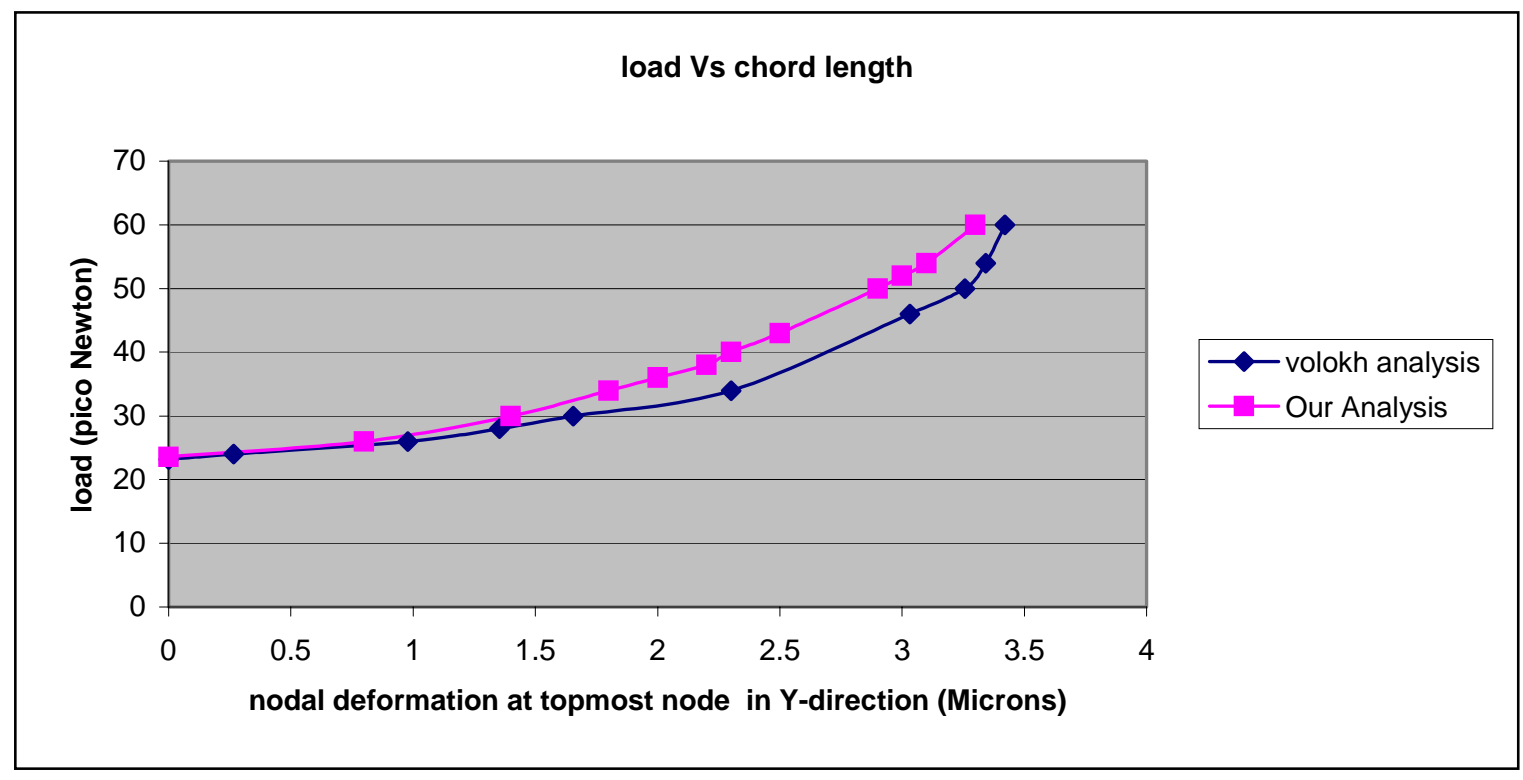

Figure 20 comparision of post buckling results of ABAQUS with elastica solution.

Comparision of our post critical analysis with that of couglin et al analysis and volokh et al analysis. Our analysis matches closely with volokh et al analysis.

\subsection{Flag Model}

\section{Post buckling of analysis for a Flag with NO-COMPRESSION condition for the lateral elements}

In a cell, the microtubule is interconnected by actin filaments, which act as lateral constraints trying to stabilize the micotubule from buckling. In a simplest level of interaction between microtubule and actin, the model can be considered like a flagpole, where microtubule is the long column (flag pole), held in place by actin ropes. The buckling behavior is studied for such a model as this case can be further extrapolated to our 3D cell model.

A riks analysis is performed for a model as shown in fig-21. Like before, 2 noded beam elements (element type B31) were used to model the microtubule and actin filaments. A No- 
Compression option was used for the actin filaments to simulate the rope like behavior of the filaments. The properties and dimensions were taken from the literature as described earlier. Pinned boundary conditions were applied to the bottom nodes of the actin elements. Perturbation and compressive loads were applied and post-buckling behavior of the microtubule column was studied like before.

Figure 21 Flag model with lateral cables Lateral elements with rope like condition (NoCompression option). A imperfection load is applied to start the buckling process. The applied to buckle the column

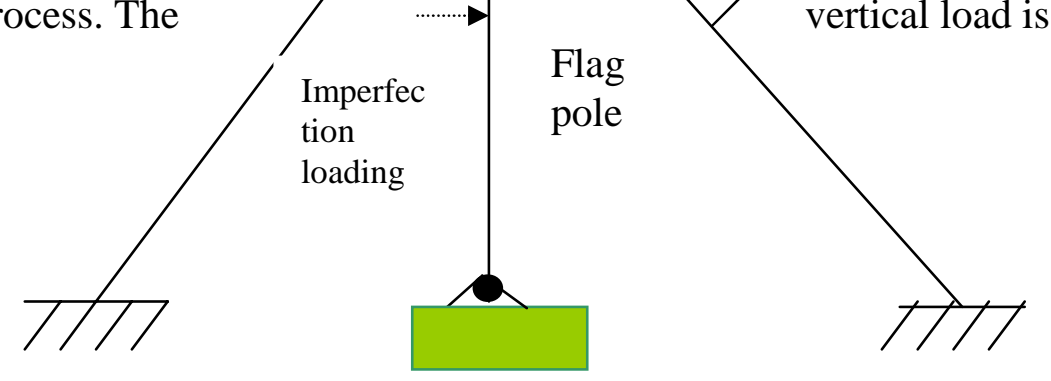

A post-buckling graph was plotted between compressive load applied Vs lateral deformation (ie direction perpendicular to the column) for the middle node in the microtubule column. Fig-22 shows the difference in the post buckling behavior of the flag model if the lateral constraints were considered as rods (can take compressive loads) instead of ropes. As expected when the lateral constraints were rods it post-buckling stiffness was much higher than when actins being considered as rope-like (No-compression). 


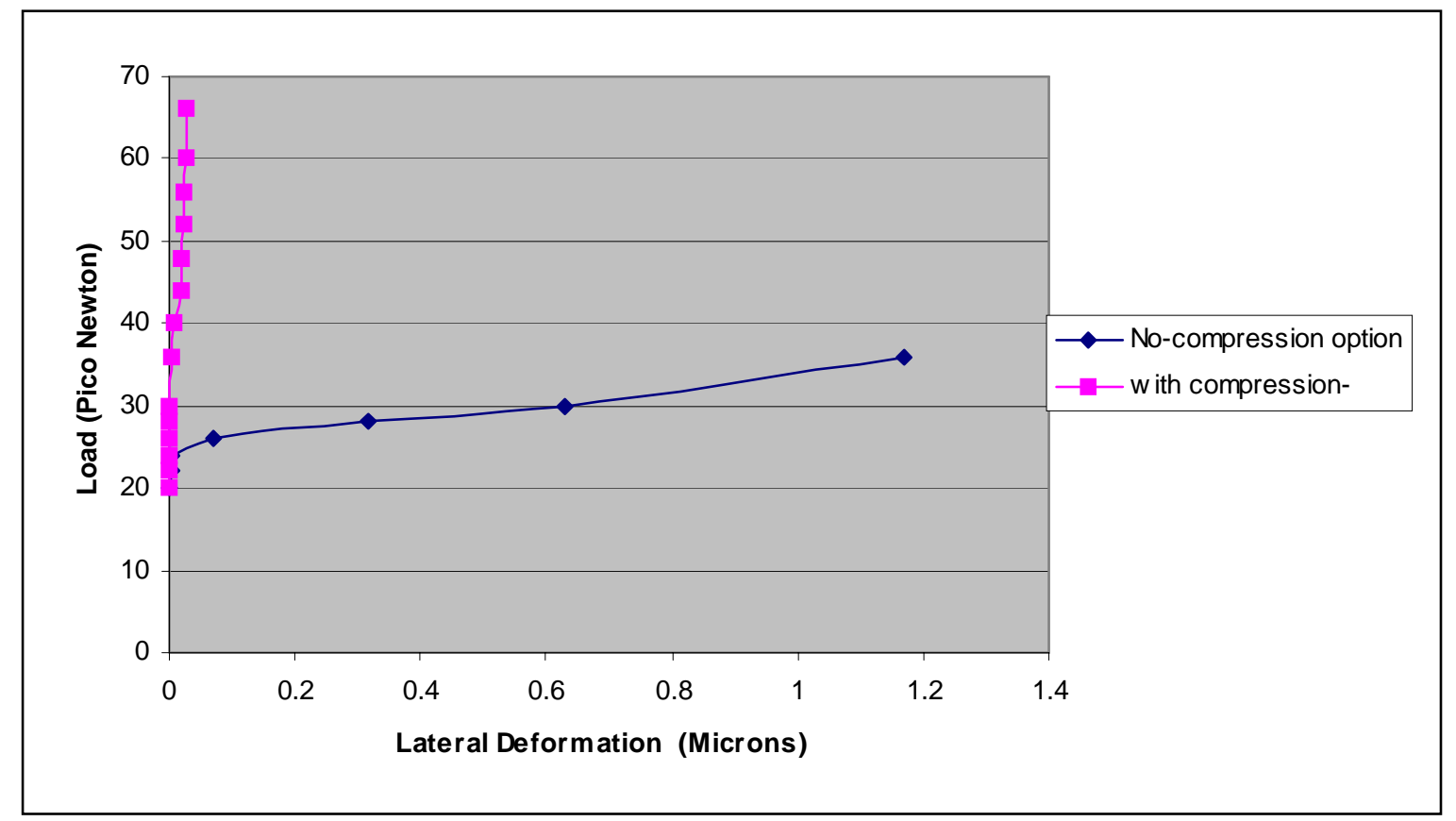

Figure 22 comparision of postubuckling of flag model with rope like lateral elements Vs rod like lateral elements.

A rope like lateral elements buckles much more than that of rod like lateral elements in flag model.

\subsubsection{Prestress Effect on Flag model}

The effect of pre-stress on the post-buckling behavior was studied. A tensile prestress was applied to the actin filaments and this caused the microtubules to be in precompression. A graph is plotted between the applied compressive load Vs lateral deformation for the middle node of the microtubule column. Like before, a typical post buckling behavior is seen above a critical load for all cases. The level of pre-stress in the actin filaments affected the post-buckling behavior of the microtubules. Simple adding the actin filaments to the free end of the microtubule column stabilized it as shown in Fig-21. A small amount of prestress $(0.001 \mathrm{MPa})$ increased the critical buckling load and also made a more stable postbuckling mode (smaller lateral deformation for a given load). However, above a certain value 
of pre-stress $(0.04 \mathrm{MPa})$, the microtubule became more prone to buckling. This finding is in agreement of the eigen value analysis performed in chapter 4.3 where the presence of a dead load reduced the active load needed to buckle a microtubule. In the riks analysis a high enough pre-stress in the actin elements produces a greated pre-load on the microtubule column thus causing it to buckle more.

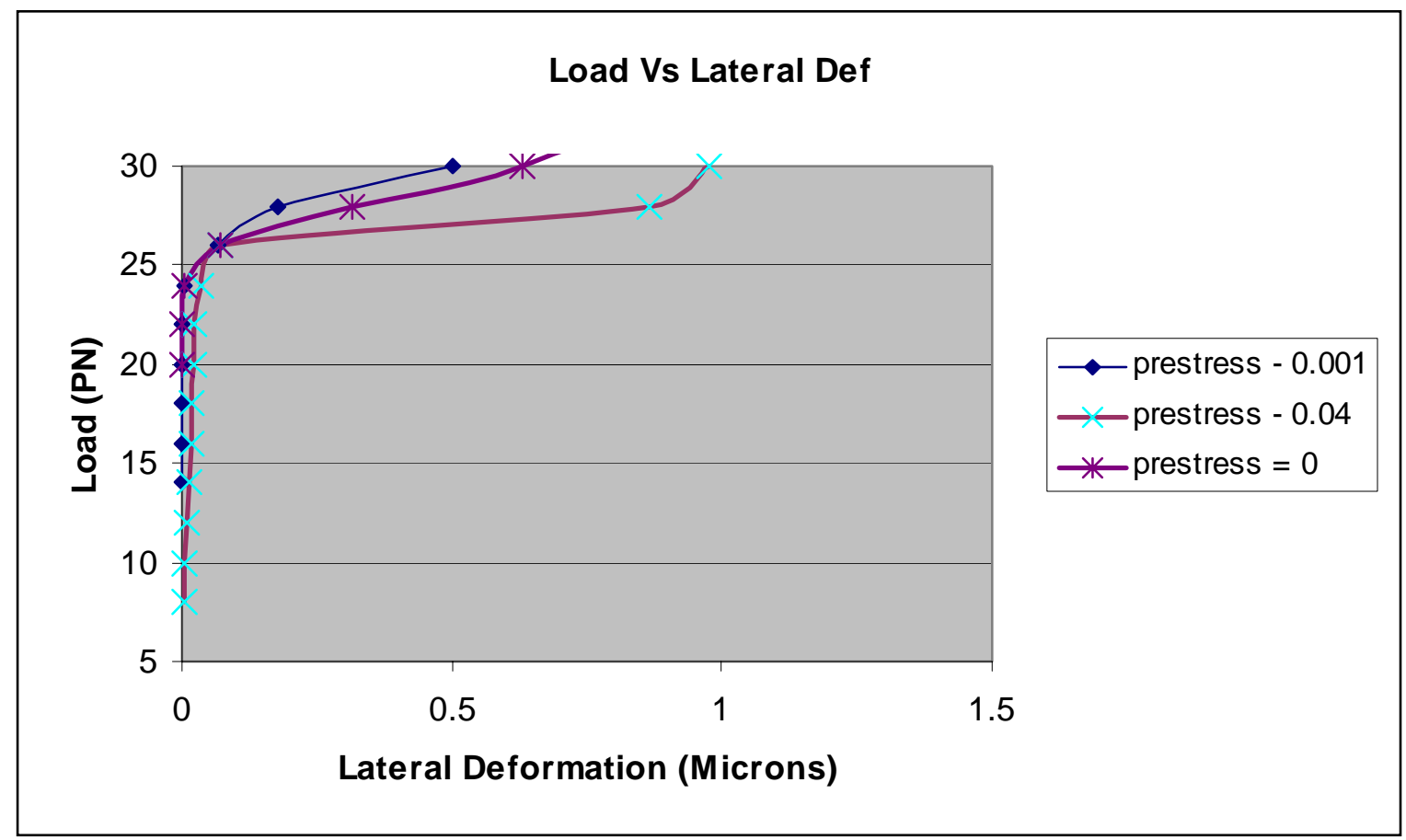

Figure 23 Effect of prestress on the pre and post buckling behavior of the flag model As the prestress is increased the deformations decreases due to stiffer boundary conditions and then the model deforms more due to the domination of applied prestress.

\subsection{Discussion}

The buckling behavior of a microtubule column was first studied. The critical load for a column with pin-ended boundary conditions and microtubule material properties is $23.57 \mathrm{PN}$. The critical load predicted by ABAQUS also matched closely with the buckling load calculated theoretically. The post-buckling behavior of microtubule column between our 
analysis and that performed by volokh et al., [42] also matched closely. The presence of actin filaments attached to the free end of the microtubule column stabilized the column. The level of pre-stress added to the actin filaments affected the post-buckling behavior of the microtubule. A small pre-stress of $0.001 \mathrm{MPa}$ increased the stability of the microtubule in the post-buckling mode. However, above a certain pre-stress value (e.g. for pre-stress=0.04 $\mathrm{MPa}$ ), the microtubule became more prone to buckling. This confirmed our expectation that the presence of a slight amount of pre-stress in the actin filaments would stabilize the free end of the microtubules. However, it puts the microtubule in compression, which increases its propensity to buckle. Thus there would be some value of pre-stress below which we would expect the stabilization but above which we would expect loss of stability. The finite element analysis showed such trends, which intuitively makes sense. 


\section{Chapter 5: Construction of a layered Finite Element Model of a Cell}

\subsection{Introduction}

Having checked the buckling behavior of microtubules when stabilized by actin filaments in a simple set up of flag like model, the next step was to put to-gether all the structural elements that contribute to the stability of a cell. These include, pre-stressed actin and microtubules in a $3 \mathrm{D}$ setup, a cell membrane and presence of internal fluid pressure. Each structural element was added layer by layer. The distribution of stress as each layer was added was studied to see if they matched with intuitive expectations.

\subsection{Materials and Methods}

A 3D finite element model of a cell was created in ABAQUS that resembled tensegrity like structure. First only cytoskeletal elements were modeled without any membrane elements. The model is shown in Fig-21. The model consists of microtubule elements emanating radially from the center of cell to the outer surface of the cell. The crosslinks represent the actin filaments that have a rope like condition by using No-Compression option in ABAQUS. Beam elements (Element type B31) were used to model microtubule and actin filaments. 
Figure 24 Schematic representation of layered addition of structural elements

Step -1 cytoskeletal structure with actin in pre-tension and microtubules in precompression, step-2 A membrane element flapped over the cytoskeletal model so that the membrane takes up some of the stresses. Step-3 Internal fluid pressure trying to blow up the entire model.

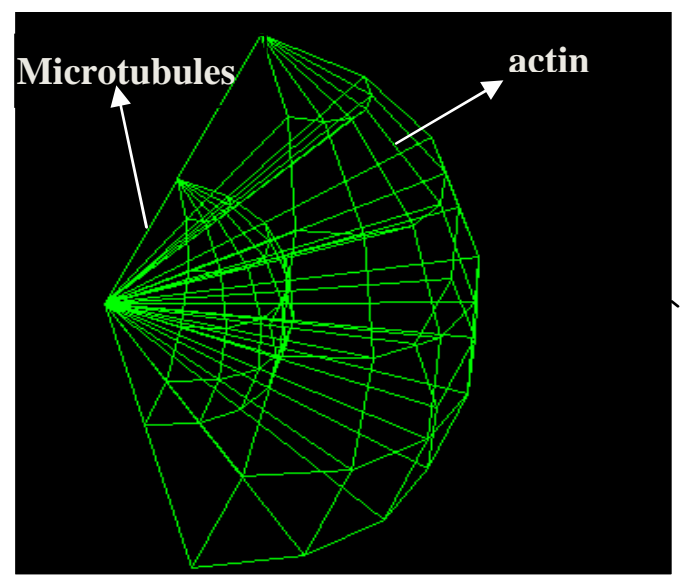

Step 1 - Cytoskeletal model, actin in pretension, microtubule in pre-compression

Step 2 - Membrane element flapped over the cytoskeletal model.
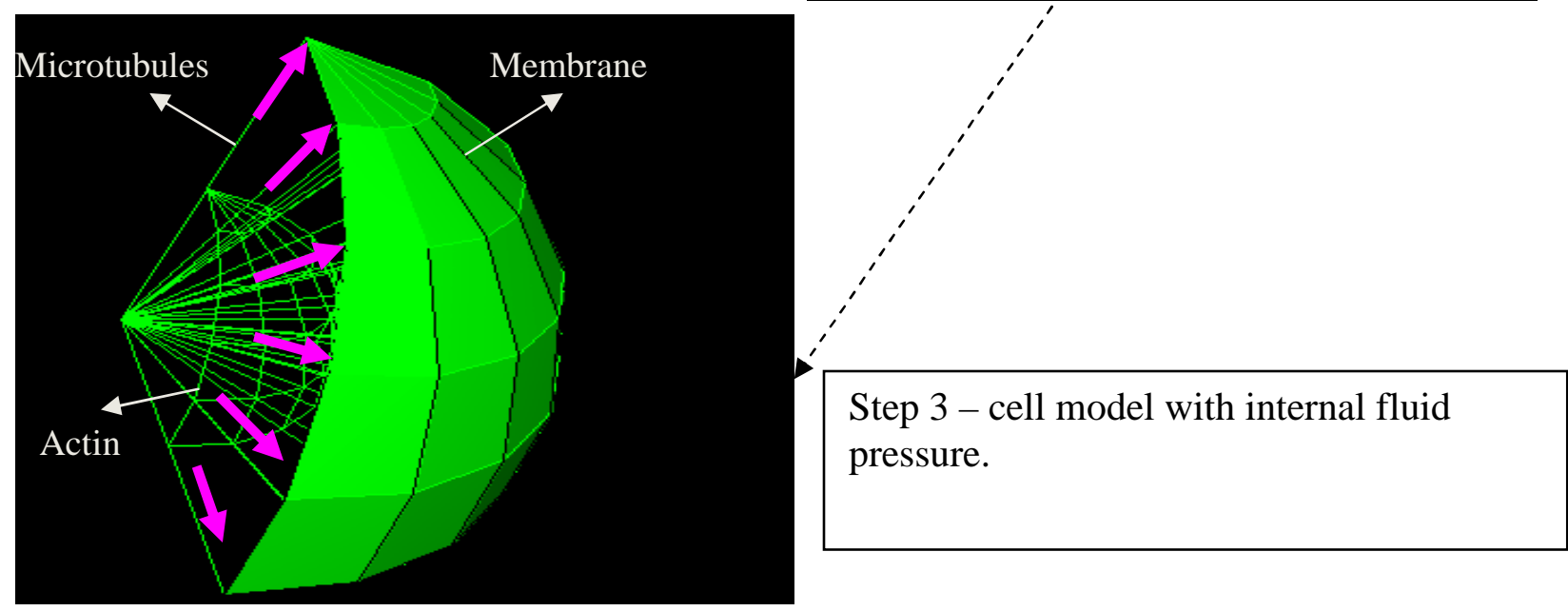
A tensile pre-stress was applied to actin filaments and this caused the microtubules to be in pre-compression. A rope like material condition was applied to the actin filaments using NoCompression option in ABAQUS A layered addition of structural elements causes change in distribution of stresses among the structural elements of the cell. First a membrane (modeled such that it can take only tensile stress and no compressive stresses) was flapped over the cytoskeletal model; it caused a change in distribution of the stresses in the cytoskeletal elements. Next, internal fluid pressure caused the whole cell to blow up and also the tensile stress in all the structural elements increased. The procedure is schematically shown in Fig21

\subsection{Results}

First a tensile pre-stress of 4.12 Mpa was applied to actin filaments. This caused the microtubules to be in compression of $3.2 \mathrm{Mpa}$. The results are shown in table-5. Next a membrane (element type M3D4) with No-Compression option was added to the cytoskeletal model, this resulted in membrane taking up some of the tensile stresses from the actin. So the tensile stress in actin reduced from 4.12 Mpa to 3.6 Mpa while the compressive stresses in microtubules increased from 3.2 Mpa to 3.5 Mpa. When an internal fluid pressure was applied, the whole model blows up and this increases the tensile stress in all the structural elements. So the compressive stresses in microtubules decreases to 0.6 Mpa while the tensile stresses in actin and membrane elements increased. 


\begin{tabular}{|c|c|c|c|}
\hline & $\begin{array}{c}\text { Microtubule } \\
(\mathrm{Mpa})\end{array}$ & $\begin{array}{c}\text { Actin } \\
(\mathrm{Mpa})\end{array}$ & $\begin{array}{c}\text { Membrane } \\
(\mathrm{Mpa})\end{array}$ \\
\hline CSK only with prestress & -3.2 & 4.12 & \\
\hline +Membrane & -3.5 & 3.6 & Negligible \\
\hline $\begin{array}{c}\text { +Membrane+ } \\
\text { Internal pressure }\end{array}$ & -0.6 & 6.2 & 0.00043 \\
& & & \\
\hline
\end{tabular}

Table 5 The stress on structural elements layered addition of each component.

Tensile pre-stress on actin causes microtubule to be in pre-compression. Addition of membrane on this model causes a reduction in tensile stress on actin. Internal fluid pressure causes increase in tensile stresses in all the elements.

\subsection{Discussion}

First the cytoskeletal (Actin and Microtubules) was modeled and a pre-tension introduced into actin network. This caused the corresponding distribution of the stresses in the cytoskeletal structure. Next when the cell membrane was added to the cytoskeletal structure, the tensile stresses were re-distributed to the actin and membrane elements. Therefore the tensile stresses in actin filaments decreased. This indicated that the membrane element was sharing some of the pre-stress of actin filaments. When the internal fluid pressure was applied, the cell expanded causing the compressive stresses in microtubule to decrease and tensile stresses in Actin and cell membrane increased. Next, a small 
perturabation load was applied to the microtubules so that they became prone to buckling. This is what we refer to as a resting state of the cell., where microtubules, prone to buckling are in pre-compression, actin and the cell membrane are in pre-tension and there is an internal fluid pressure. The distribution of stresses as each structural element is added makes sense intuitively. Finally a perturbation load was applied to each microtubule to make them prone to buckling under compressive load. This representated the resting state of the cell, where the microtubule were in pre-compression, the actin and cell membrane were in pretension, there was an internal fluid pressure and the microtubule prone to buckling. 


\section{Chapter 6 -Buckling of microtubules in a 3D cell model}

\subsection{Introduction}

Exploring the literature, three mechanisms by which cells can resist compressive loading were identified. One of them is, that microtubules are the compression bearing elements in a cell $[30,33]$. In specific cells, when the microtubule elements were dissolved, the whole cell reduced in volume, which indicated that, the microtubules probably resisted compression [33-35]. In separate experiments, the transfer of force to a stretchable membrane on which the cells were grown was measured when the microtubule network was dissolved. These also indicated that the microtubules were in compression.[30, 33, 35]. Direct visualization experiments show that microtubules can buckle inside a cell ostensibly due to compressive loading[30]. Microtubules have a very high slenderness ratio (Length $=7.5 \mu \mathrm{m}$, diameter $=25 \mathrm{~nm}$, thus slenderness ratio $=300$ ), thus making them prone to buckling. However, the inter connections with other cytoskeletal elements would prevent a catastrophic failure of the microtubules. We thus incorporated buckling of microtubules and modeled the post buckling behavior of the microtubules in our model.

\subsection{Materials and methods}

In chapter 6 a simple flagpole model of cytoskeletal architecture was validated. In this chapter we put together the model of the whole cell. A finite element model of a cell was developed using as shown in fig-22. The cell was modeled as having a spherical shape. Taking advantage of the symmetry of the geometry of the cell, only one-eighth of the cell was modeled with the coordinate planes $x-y, y-z$ and $z-x$ acting as planes of symmetry. 
Boundary conditions consistent with the symmetry were applied (nodes in a plane of symmetry were not allowed to have displacements in a direction perpendicular to the plane, i.e points in the $x y$ plane were not allowed to displace in the $\mathrm{z}$ direction etc.). An idealized cytoskeletal structure was chosen. The microtubule network was modeled with 2-noded beam elements (element type B31) emanating from the center of the cell to end on the cell membrane. The actin network was transverse to the microtubule network and was modeled with 2-noded beam (element type B31) elements joining adjacent microtubule filaments - at the outer membrane as well as various radial distances from the center of the cell (fig 1 shows a case where only the center of the microtubule elements are joined). The cell membrane was meshed with 3 and 4 noded membrane elements (element type M3D4 and M3D3).

Figure 25 3D finite element model of a cell

Microtubules emanating from the center to the cell membrane. Actin filaments forming the cross mesh, stabilize the microtubules from buckling

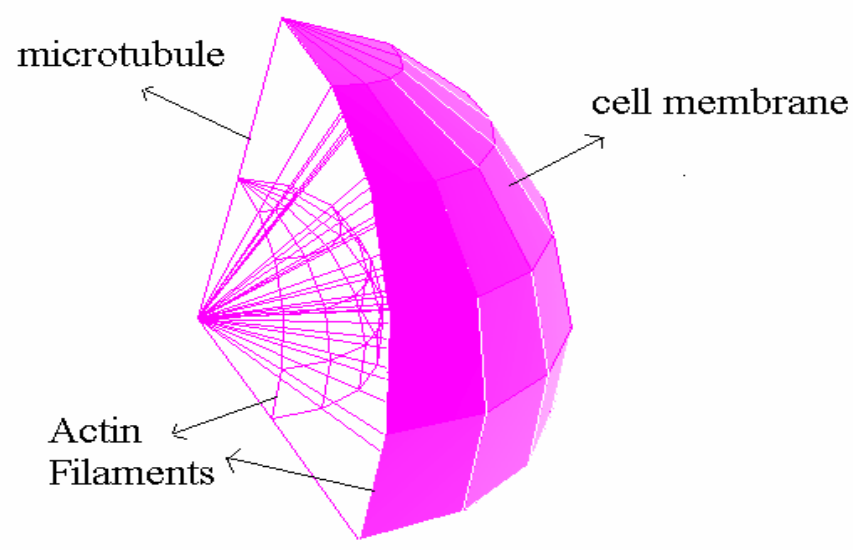

An analogy can be drawn to a tent, where the microtubules are the main masts of the tent, the ropes are the actin filaments, the tent material is the cell membrane and the air inside is pressurized.

The properties and dimensions of the various components of the cell were taken from the literature. Based on measurements of the size of prechondrocytic ATDC5 cells embedded in agarose gel in our laboratory, we chose the radius of the cell as 7.5 micrometers. The 
modulus of elasticity of the cell membrane was taken to be $0.1 \mathrm{MPa}$ and its thickness to be 0.01 microns|. This was based on values obtained from experiments on red blood cells by Hochmuth et al [19]. The no compression option in ABAQUS was used for membrane elements, to prevent the membrane from supporting any compressive loads. Studies on microtubule structure reveal that they are like hollow cylinders of $25 \mathrm{~nm}$ outer diameter and thickness of $2.7 \mathrm{~nm}$ while the actin filaments are ropelike and are $8 \mathrm{~nm}$ in diameter [20]. From this, the cross sectional moment of inertia (I) of these elements could be calculated. Gittes et al., [22] measured the flexural rigidity (EI) of microtubules and actin filaments to be 2.1e-23 Nm $\mathrm{Nm}^{2}$ and 7.3e-26 $\mathrm{Nm}^{2}$ respectively. Thus, the Young's modulus (E) for the actin and microtubule filaments was calculated to be $1.2 \mathrm{e} 03 \mathrm{MPa}$ and $3.63 \mathrm{e} 02 \mathrm{MPa}$ respectively.

The microtubules of the cell are stabilized by the presence of the pre-stress in the actin filaments- thus the actins are in pre-tension and microtubules in pre-compression, consistent with studies on these cytoskeletal structures. To model the buckling of microtubules in cell model, a small perturbation load $(0.01 \mathrm{pN})$ was applied to the microtubule elements in a direction perpendicular to the axis of each microtubule element and at its midpoint. This perturbation load made the microtubules prone to buckling under compressive load. In ABAQUS the perturabation load needs to be resolved into there respective $\mathrm{x}, \mathrm{y}$ and $\mathrm{z}$ components. The direction cosines perpendicular to the microtubule were found. The actual perturbation load was multiplied by the direction cosine to resolve the load into there respective components. The procedure resolving the perturbation load into their respective components is described in APPENDIX-7.

Next we applied a point load the cell model and looked for the buckling behavior of the nearby microtubules. The point load was applied to a outer node (at the membrane) in the 
direction along the axis of the microtubule. This load is also resolved into the respective components. The procedure for resolving the point load is described in APPENDIX-7. The effect of different amounts of prestress in the actin cytoskeleton was examined. Also whether the cytoskeletal structures far away from the point of application of the point load were being affected (action at a distance).

\subsection{Results}

\section{Buckling of microtubule in a cell subjected to point load:}

The cell model is shown in Fig-22. A point compressive load (upto $100 \mathrm{pN}$ ) was applied at the outer node of a microtubule in the direction along the axis of microtubule element. Like before, a RIKS procedure was used in ABAQUS to perform the post-buckling analysis of all microtubules in the cell. Fig-26 shows the deformation of the microtubules in the cell in response to the applied point load. The microtubule, which was loaded showed buckling behavior, thus replicating cell behavior to point loading/perturbation, aas seen in certain experimental setups [30]. Fig-26 shows the post buckling plot between the applied load and lateral deformation (deformation in the direction of perpendicular to axis of microtubule for middle node of the microtubule to which load is applied) of the middle node of the 


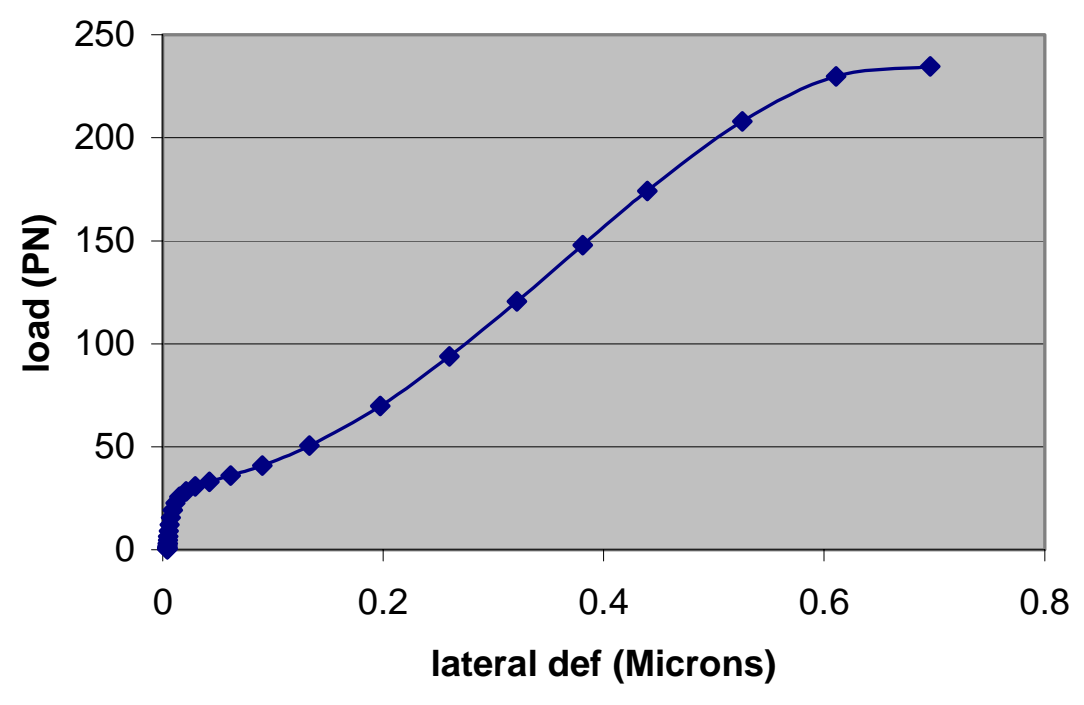

Figure 26 Post-buckling of microtubule in a cell.

The plot shows the lateral deformation of microtubule at the center Vs the applied compressive load. The microtubule begins to buckle at around 35 pico-newton

microtubule to which the load is applied. An ABAQUS subroutine was written using FORTRAN to extract the lateral deformation of the middle node for every load increment. Fig-23 shows the plot between the (load factor * actual compressive load applied) to microtubule Vs lateral deformation. The FORTRAN program for extracting the lateral deformation for each load increment is given in APPENDIX-8. Fig-26 shows that microtubule in a cell starts to buckle at around $35 \mathrm{pN}$. This is slightly higher than $23.57 \mathrm{pN}$, which is the predicted buckling load for a single microtubule. The increase in buckling load is expected because several actin filaments stabilize this microtubule. The presence of actin filaments as lateral constraints also increased the post buckling stability of the microtubule. We next checked the effects of different amounts of prestress in the actin elements on post buckling stability of the microtubule to which the load was applied. 


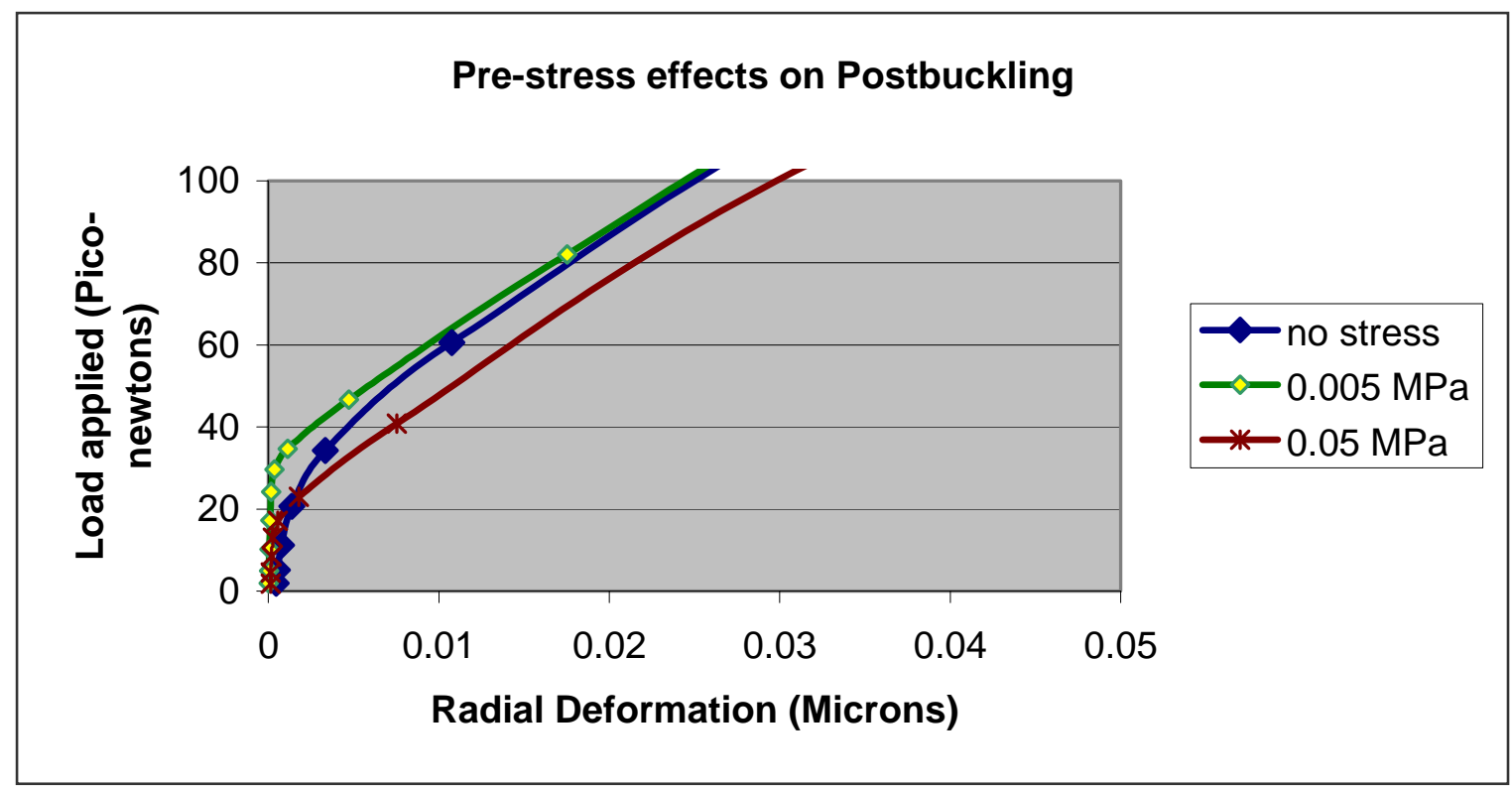

Figure 27 Effect of pre-stress of actin filaments on the post-buckling stiffness of the cell. Without pre-stress the microtubule starts to buckle at a load of around $35 \mathrm{pN}$, while a slight increase in prestress to $0.005 \mathrm{MPa}$ causes the microtubule to stabilize and buckle at $38 \mathrm{pN}$. While further increase in prestress to $0.05 \mathrm{MPa}$ enhances the buckling behavior decreasing the stability. So the microtubule buckles at around $28 \mathrm{pN}$.

When a small tensile pre-stress of $0.005 \mathrm{MPa}$ (correspond load of 0.25 Pico-newtons applied on actin filaments) was applied to actin filaments, it increased the post buckling stability (Fig-27). The post-buckling curve showed that for a given load, the deformation is lesser than the case for which no pre-stress was applied. But as the tensile pre-stress was increased to 0.05 $\mathrm{MPa}$ (corresponding to 2.5 Pico-newtons applied to actin filaments) the deformations increased, indicating decreased post-buckling stability. Therefore pre-stress in actin filaments plays a role in the post buckling stability of microtubules in a cell. This trend was similar to the effect of prestress observed in the 2D flag model we studied earlier, although the actual values of prestress which caused similar responses was higher in the cell model. This is 
expected since the microtubule in the cell is much more constrained than in the flagpole model.

\section{Action at a distance}

Since the structural components of a cell are interconnected, it is intuitively reasonable that mechanical perturbation at a particular point in the cytoskeleton would have some effect at locations in the Cytoskeleton that are remotely located from the point of perturbation. This general phenomenon is referred to as action at a distance. Several instances of this have been experimentally shown in various experiments. Special junction complexes consisting of integrins couple the Extracellular matrix (ECM) of a cell to its cytoskeleton (e.g. actin). Experiments show that when a pulling load was applied to such adhesion complexes, it would cause the cytoskeletal elements to reorganize in the direction of the load applied [32].

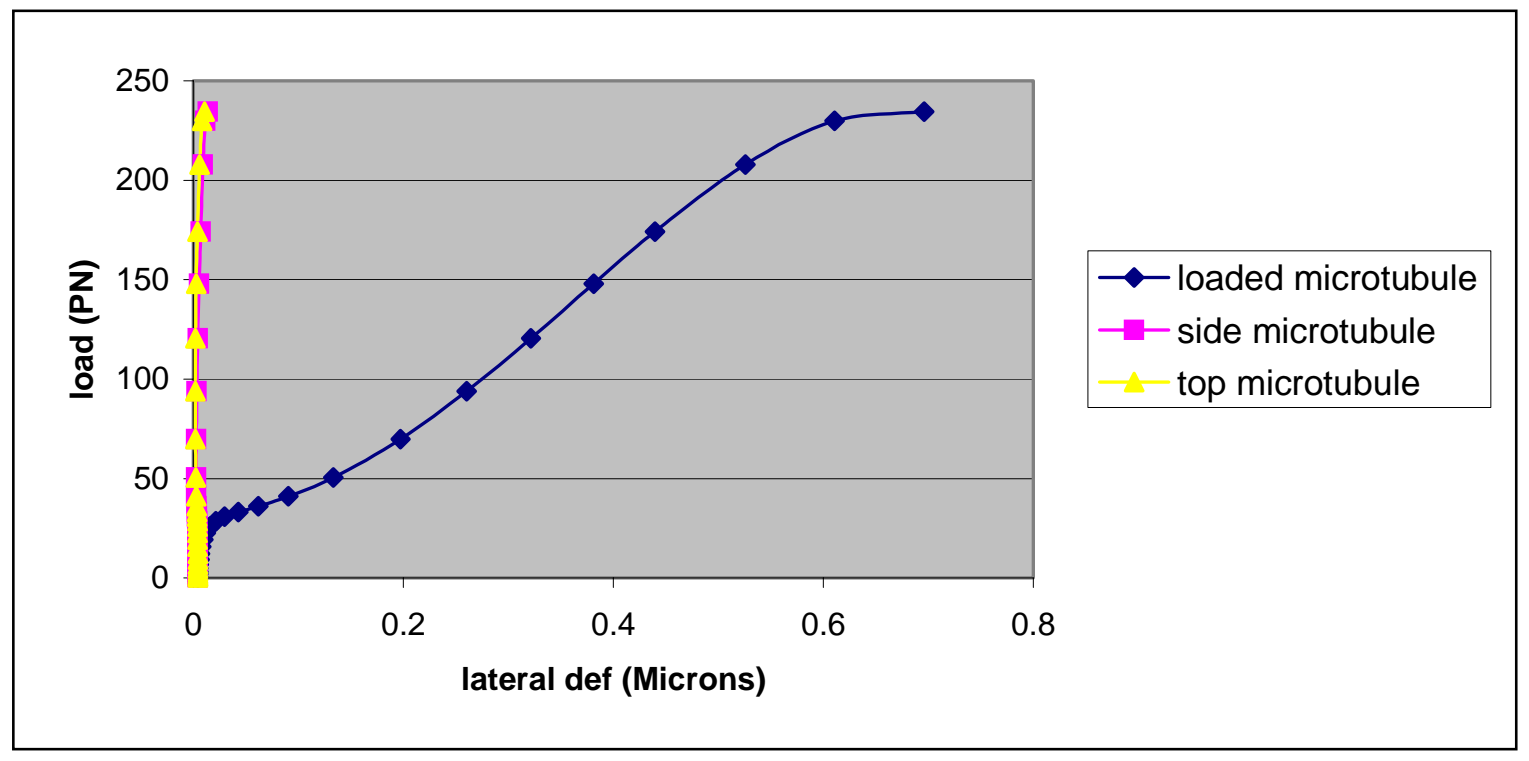

Figure 28 Post-buckling behavior of the neighboring microtubules

Post-buckling behavior of the neighboring microtubules when a load is applied to a single microtubule. The plot shows the lateral deflection of the neighboring microtubules at the mid point Vs the load applied the actual microtubule. 
Similarly, point loads applied to portions of the Cytoskeleton caused significant movement in the cell nucleus that was located far away from the point of loading. We wished to see if Similar phenomenon was observed for our cell model. When a point compressive load was applied to an outer node of a microtubule, as shown in Fig-28, it caused the microtubule to buckle. But as the load was further increased, it led to deformation and eventual buckling of distant microtubules as well. Fig-30 shows the picture of cell where in not only does the neighboring microtubules buckle but also microtubules at a distance also buckles. In our model, it appears that an ordered progression of the buckling occurs: the microtubule that is directly loaded begins to buckle first, then by virtue of the connecting actin elements, the neighboring microtubules begin to buckle, and even remotely placed microtubules begin to buckle.

A similar event occurs when a tensile load (100 PN) is applied to a single microtubule in the cell. Fig 29 shows that when a point pulling load was applied, it cause the microtubules at a distance to buckle. There is large deformation in microtubules located far away from the loaded microtubule. This shows that our model exhibits the phenomena of action at a distance.

Figure 29 Action at a distance- a pulling load applied at a point.

A pulling load of 100 piconewton causes the neighboring microtubules to buckle and also microtubule at a distance to buckle.

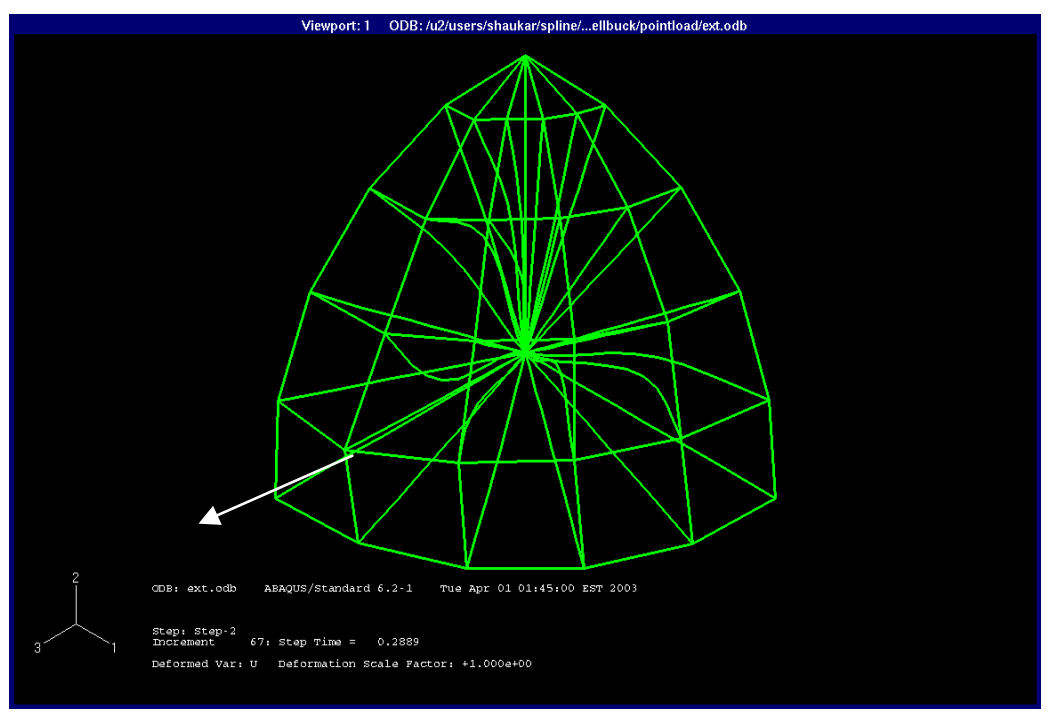


Figure 30 Action at a distance - microtubule in a cell subjected to compressive point load.

The figure shows that when a compressive load is applied to a microtubule, even the neighboring microtubules bucking and also microtubules at a distance also tend to buckle.

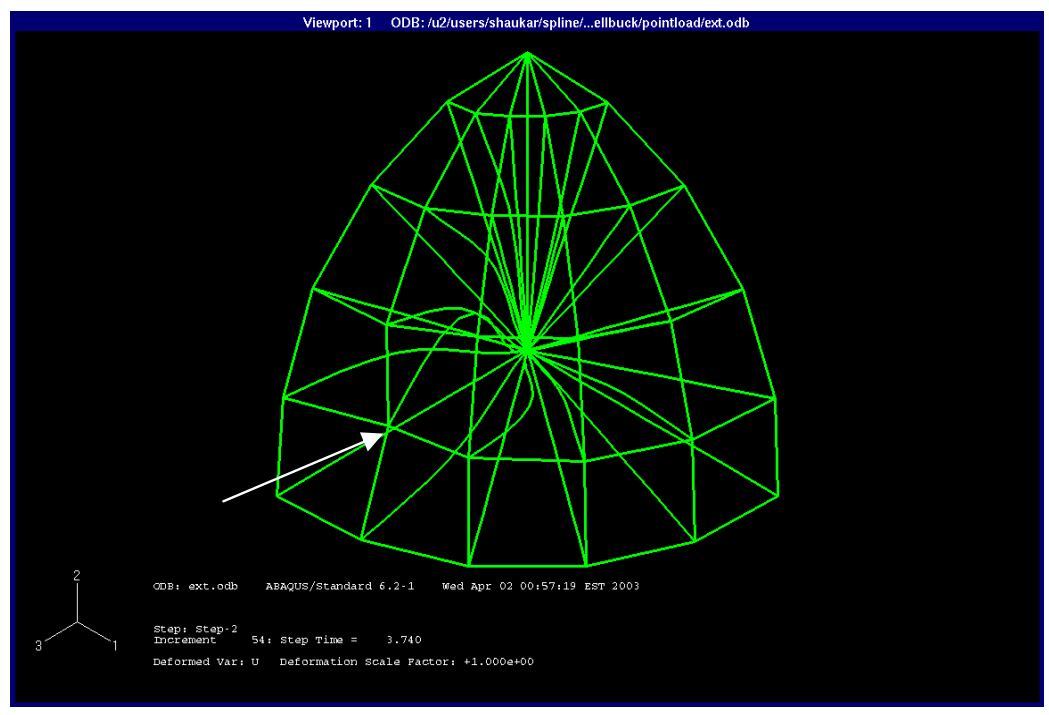

\subsection{Discussion}

The buckling behavior of the microtubules in a 3D cell architecture was studied. The structural organization of microtubule and presence of actin filaments stabilizes the microtubules prone to buckling. when a microtubule is considered as a single column it buckled at 23.57 PN while the same microtubule in a cytoskeletal architecture of a cell, buckled at a much higher load of $35 \mathrm{PN}$. Thus the presence of actin filaments and the way in which they are organized affected the stiffness of the microtubule and also the overall stiffness of the cell. Not only did the presence of actin filaments affect the overall stiffness, even the pre-stress in actin filaments affected the stiffness of microtubules prone to buckling. A small pre-stress $(0.005 \mathrm{MPa})$ increased the critical buckling load and also made the microtubule more stable in post buckling mode (ie smaller lateral deflection for a given load). However beyond a certain pre-stress $(0.05 \mathrm{MPa})$, the microtubule became more prone to buckling. This showed that for a slight prestress would stabilize the free edge of the microtubule. However, further increase in the pre-stress puts more compression on the microtubule thereby increasing its propensity to buckle. Action at a distance is a phenomena 
when a pulling load is applied to special juction complexes, it would cause all the cytoskeletal elements to reorganize in the direction of load applied. This action at a distance has been experiementally verified. Our cell model also showed a similar phenomenon where in when a point compressive load was applied to a outer node of a microtubule, it caused the neighboring and other microtubules also to buckle. As the load was increased further, it caused the microtubule at a distance also to buckle. This showed that the model could also predict action at a distance phenomenon that has been verified experiementally. Similar phenomena of action at a distance was seen when our cell model was subjected to a pointpulling load. This caused the microtubules at a distance also to buckle. Such a validated and tested model that includes all the structural features would be useful in studying the various aspects of mechanical environment of cell. This would form a beginning for testing various conditions and verifying with the experimentally observed features. 


\section{Chapter-7: Uniaxial loading of 3D Finite Element Model of cell}

\subsection{Introduction}

Once the model was able to predict certain expected reactions to point loads, we extended our area of investigation to compressive loading. Related experiments in our laboratory aim to explore the deformation of chondrocytes and prechondrocytic cells in response to compressive loading regimes. The cells are embedded in agarose gel and the cellgel construct is compressed. The cells are fluorescently labeled, so that the deformations of the cell to applied loading can be measured under a confocal microscope. We are able to determine mechanical properties of the cells from these experiments by fitting the radial deformation of the cell to a closed form solution of the radial deformation of an inclusion (e.g. the cell) in an infinite medium (e.g. the gel) obtained by Goodier, 1933 [39]. The relevant equations are given in Appendix-8. We wanted to explore, how the structural elements of the cell influence the overall material property of the cell. The finite element model was used for this purpose. For a given structure, a uniaxial compressive load was applied to the model and the radial deformations were recorded. Next these were curve fit with Goodier's solution to obtain an equivalent modulus for the cell. In this case, the material property of the infinite medium was set to zero, and the load on each node was calculated to result from a uniformly applied load on the infinite medium. This was repeated for cells with different structures, internal pressures, prestress values. We then plotted the change in the curve fit value of the cell modulus as a function of the parameters. 


\subsection{Materials and methods}

The 3D finite Element model of cell has already been discussed in previous chapters. The model consists of cell membrane, microtubules emanating from the center to the outer membrane, actin filaments acting as cross-links stabilizing the microtubule that are prone to buckling. A perturabation load is applied to all the microtubules at the mid point between the center and outer membrane. The perturabation load (=0.01 Pico-Newton) is applied in a direction perpendicular to the radial axis of the microtubules. A uniaxial compressive load was applied to all the nodes at the outer membrane of the model. This kind of loading is to simulate the condition of cell embedded in a gel subjected to uniaxial compressive loads. The radial deformation of a edge of the our model is extracted by using a ABAQUS subroutine. The ABAQUS subroutine is described in APPENDIX-8. Goodier, 1933 [39] found a closed form solution for a spherical inclusion embedded in an infinite medium subjected to uniaxial compressive loading. The representative model is shown in Fig-31.

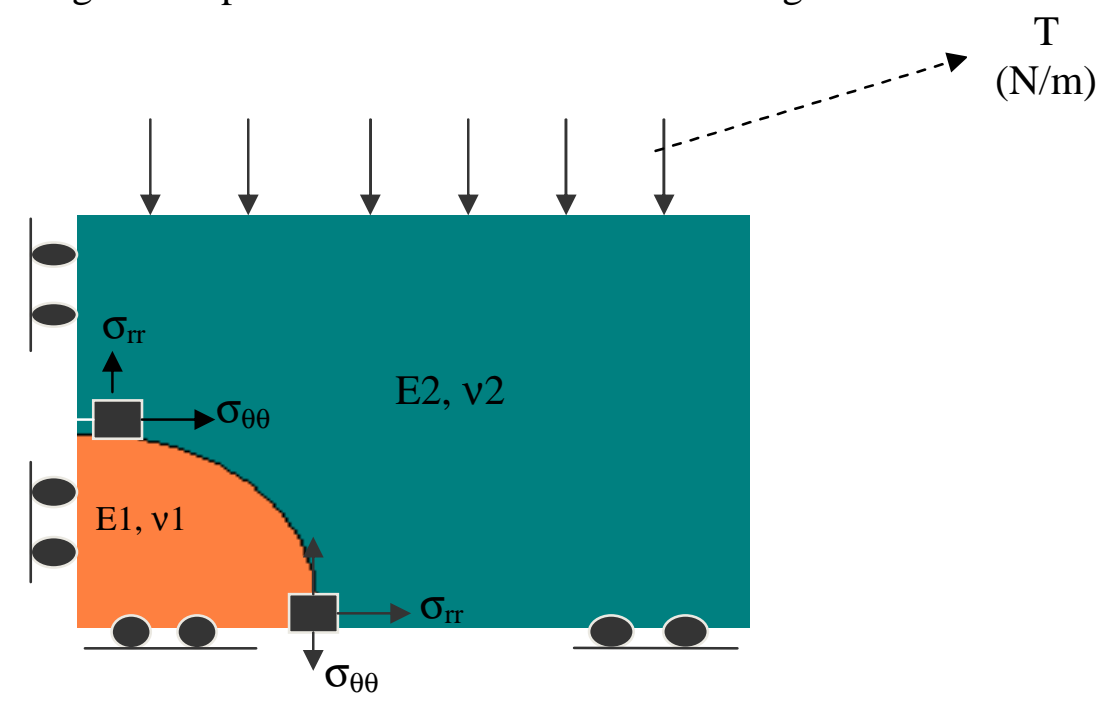

Figure 31 Goodier's model of spherical inclusion inside a infinite medium

The model is subjected to a compressive stress of $\mathrm{T}$. The inclusion has a material property of E1, $v 1$ and the medium with material property of E2, $v 2$. 
The equations for finding the radial deformation at the junction between the inclusion and the medium are

$U_{r}^{b}=-\frac{A}{r^{2}}-\frac{3 B}{r^{4}}+\left(\frac{5-4 v_{b}}{1-2 v_{b}}\right) \frac{2 C}{3 r^{3}}+\left[\frac{-9 B}{r^{4}}+\left(\frac{5-4 v_{b}}{1-2 v_{b}}\right) \frac{C}{r^{2}}\right] \cos 2 \theta+\frac{T r}{2 E}\left[\left(1-v_{b}\right)+\left(1+v_{b}\right) \cos 2 \theta\right]$

$$
U_{\theta}^{b}=-\left[\frac{6 B}{r^{4}}+\frac{2 C}{r^{2}}\right] \sin (2 \theta)-\frac{T r}{2 E}\left(1+v_{b}\right) \sin 2 \theta
$$

Where $U_{r}^{b}$ is the equation for the radial deformations at any point on the medium. There is separate set of equations for finding the deformations inside the inclusion, which is given in the Appendix-9. $\mathrm{T}$ is the compressive load applied in $\mathrm{N} / \mathrm{m}, \mathrm{r}$ is the radius of the inclusion, $\mathrm{A}$, B, C are separate set of equations representing the young's modulus of the inclusion and the medium. The equations of $\mathrm{A}, \mathrm{B}$ and $\mathrm{C}$ is given in Appendix-8. The equivalent young's modulus of the our model subjected to Uniaxial compressive loading is arrived by curve fitting the radial deformation at a outer edge of our cell model with that of the goodier's model. In goodier's model the material property of the medium is set as zero, and the radius of the inclusion is same as that used in our cell model. The uniaxial compressive load applied is equivalent to that applied to our $3 \mathrm{D}$ cell model. The equivalent load $\mathrm{T}$ is $(\mathrm{n} * \mathrm{P}) / \mathrm{r}$ where $\mathrm{n}$ is the number of nodes along the edge of the membrane, $\mathrm{P}$ is the load applied at each nodes of the membrane and $\mathrm{r}$ the radius of the cell. The schematic representation of the conversion is shown in Fig-32 

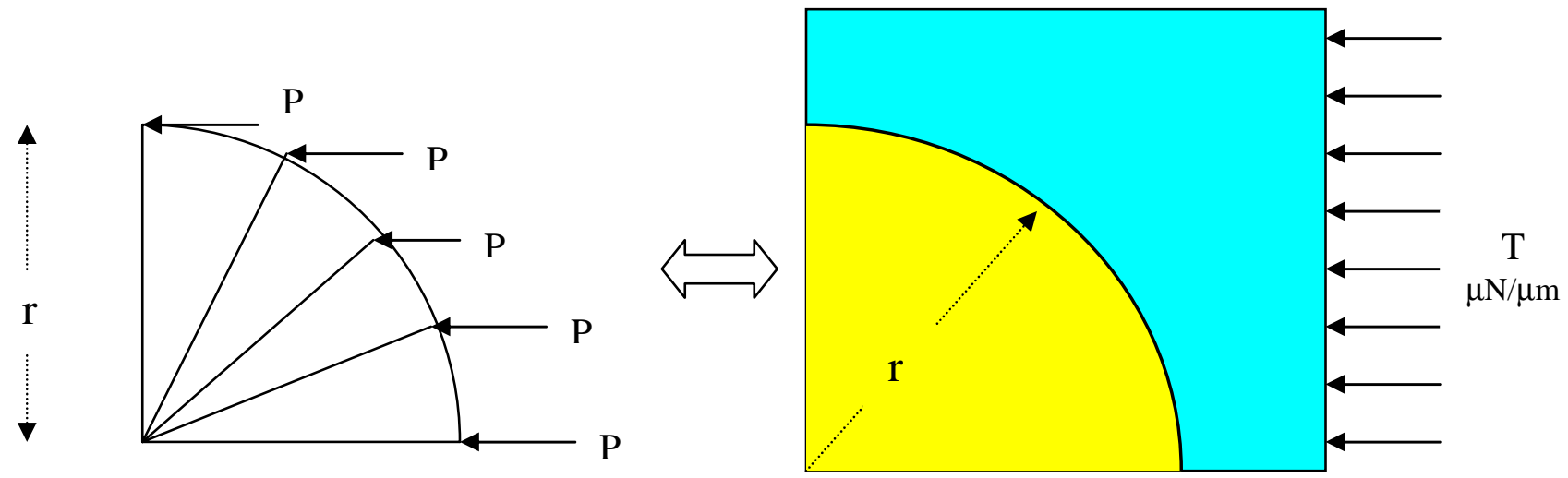

Cytoskeletal model

Continuum model

Figure 32 load required in goodier's model equivalent to the load applied to cell model

The relation between the load (in $\mu \mathrm{N}$ ) applied at n-nodes along the curved edge for the cytoskeletal model can be related as equivalent uniformly distributed load $\mathrm{T}$ for a continuum model. $\mathbf{n P}=\mathbf{T r}$

The equivalent young's modulus of the cell is found by curve fitting the deformation at the juction between the medium and inclusion with that of the radial deformation of the our cell model.

This procedure is explained diagrammatically in Fig-33. First step is to compress the cell model subjected to uniaxial loading. Next step is to record the nodal deformation along the radial direction at the edge of the membrane. Next step is to convert the equivalent point load into uniform compressive stress applied to goodier's model. Finally curve fit for the young's modulus of the cell such that the radial deformation for the goodier's model is same as that of the $3 \mathrm{D}$ cell model. 


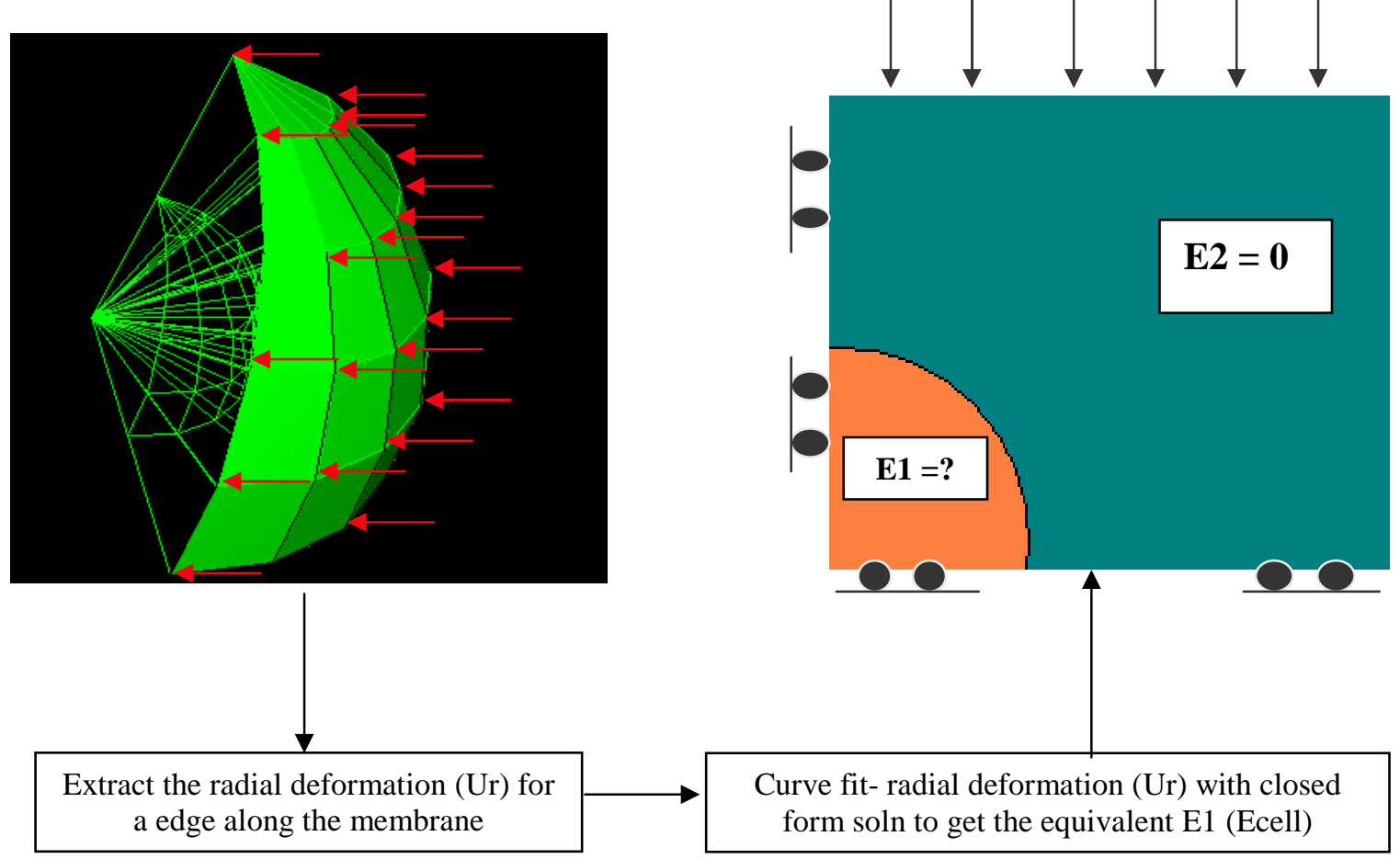

Figure 33 Schematic representation of finding the equivalent young's modulus for uniaxial loading.

From the cell model, the radial deformations are extracted using abaqus subroutine, the deformation is fit with that of closed form solution of spherical inclusion inside a medium solved by Goodier 1933.

The closeness of the fit for a representative value of uniaxial loads and material properties is shown in Fig-34. A graph is plot between the radial deformation extracted from the 3D cell model Vs the radial deformation of the inclusion with medium young's modulus $=0$. The plot shows the closeness of the fit where the deformation of the goodier's model match well with that of the 3D cell model. 


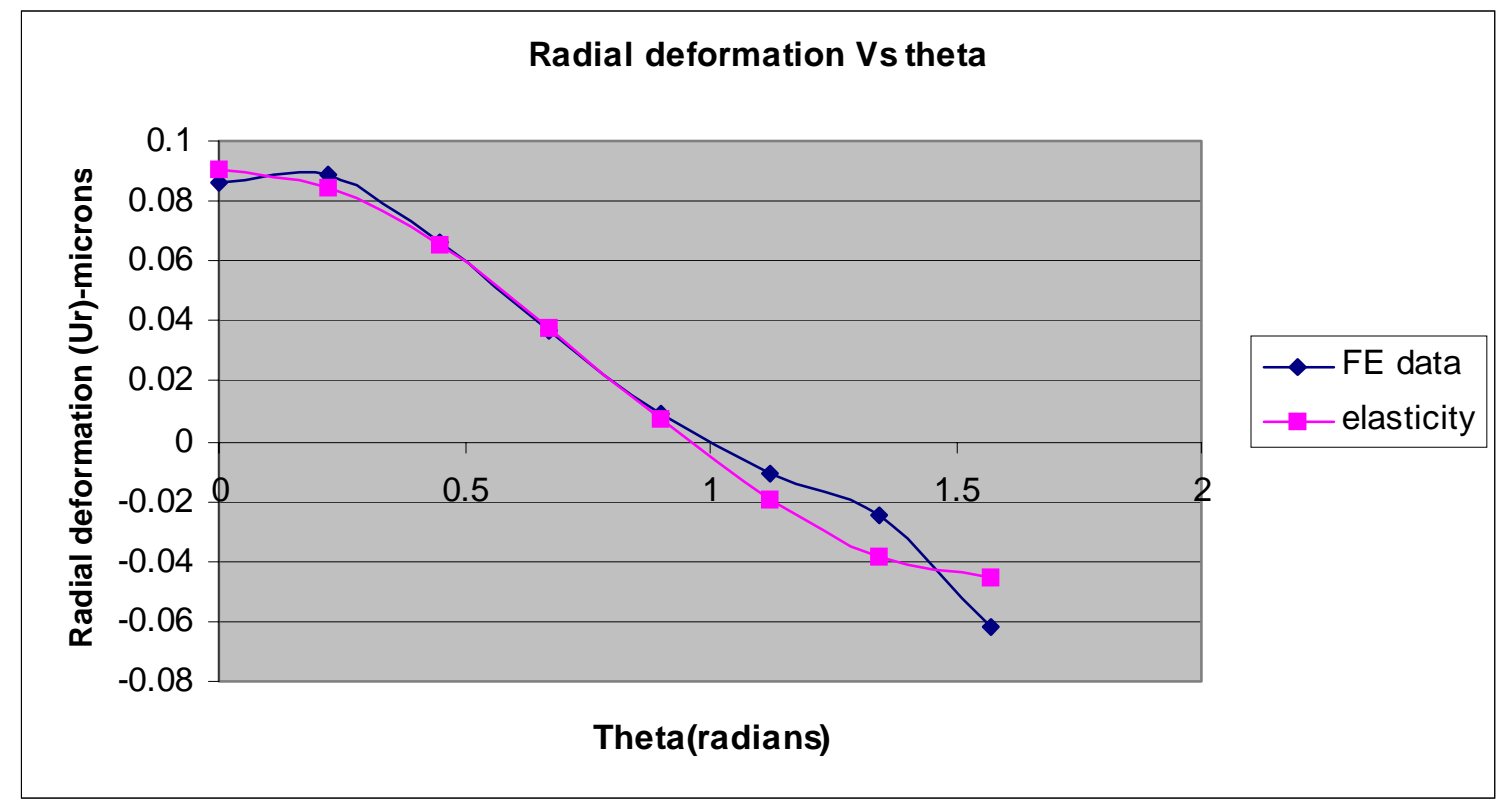

Figure 34 Closeness of Fit between the cell model Vs the goodier's closed form solution.

A plot between the radial deformation at the edge of the outer membrane Vs location at various angles where the deformation is extracted. The pink curve shows the equivalent young's modulus for which the radial deformation for the goodier's model fit well with our cell model.

This procedure is repeated for cells with different structures, internal pressure and prestress values. A parametric analysis was performed for the changes in various values of the cell and its effect of the overall young's modulus of the cell. A significant discovery of the cell model subject to uniaxial compressive loading was that it was not stable, if we used the "nocompression" option for the actin filaments. When the actin filaments were allowed to resist compression as well, it stabilized the cell model. An analysis of the stresses in the actin filaments showed that while most of the actin network was under tension, a few filaments were under compressive stresses, and apparently that is critical for the stability of the whole structure. 


\subsection{Results}

A uniaxial loading caused the cell to compress in the direction of the load and slightly elongate perpendicular to the direction of the load, as would be expected. Thus radial deformations were $-\mathrm{ve}$ in the direction of the load, but became +ve perpendicular to the direction of the load. A representative graph is shown in Fig-28. The predicted radial deformations of an inclusion embedded in an infinite medium from Goodiers solutions showed a similar trend. The equivalent modulus of elasticity of the cell for which the finite element solutions and the Goodier's solutions matched are normalized to $\mathrm{E}_{0}=24.46 \mathrm{KPa}$. A parametric study was performed to see the effect of various structural parameters on the overall stiffness of the cell measured in terms of its equivalent young's modulus. The parametric variation is made dimensionless by dividing the varying parameter with a constant values, which in our case is $\mathrm{E} 0=24.46 \mathrm{KPa}$.

The effect of variation on number of actin crosslinks on the overall stiffness of a cell is shown in Fig-33. Fig-33 shows a plot between effect of increase in number of actin crosslinks on the dimensionless E/E0 quantity.

As seen in Fig-33, A 3 fold increase in number of crosslinks causes less than 1\% increase in Equivalent young's modulus. This shows that for uniaxial loading conditions the effect of actin crosslinks on the overall stiffness is not substantial.

Fig-34 shows the effect of \# of microtubules on the equivalent young's modulus of cell. A 4fold increase in \# of microtubules caused a $6 \%$ increase in the equivalent young's modulus. This shows that the fluid pressure has a important role on the overall stiffness of the cell. 
Fig-35 shows the effect of internal fluid pressure on the equivalent young's modulus of cell. Here the internal fluid pressure is also made dimensionless by dividing the internal fluid pressure with a constant value of $0.001 \mathrm{MPa}$. A 10-fold increase in internal fluid pressure caused a $6 \%$ increase in the equivalent young's modulus. This shows that the fluid pressure has a important role on the overall stiffness of the cell.

Fig-36 shows the effect of pre-stress in microtubules on the modulus of elasticity of cell. The variation of dimensionless pre-stress on the overall stiffness of the cell is plotted. The prestress is made dimensionless by dividing the pre-stress by a constant value of $0.0005 \mathrm{MPa}$. Fig-36 shows a negligible increase in stiffness of less than $0.01 \%$ for a 1000 fold increase in applied pre-stress for a cell.

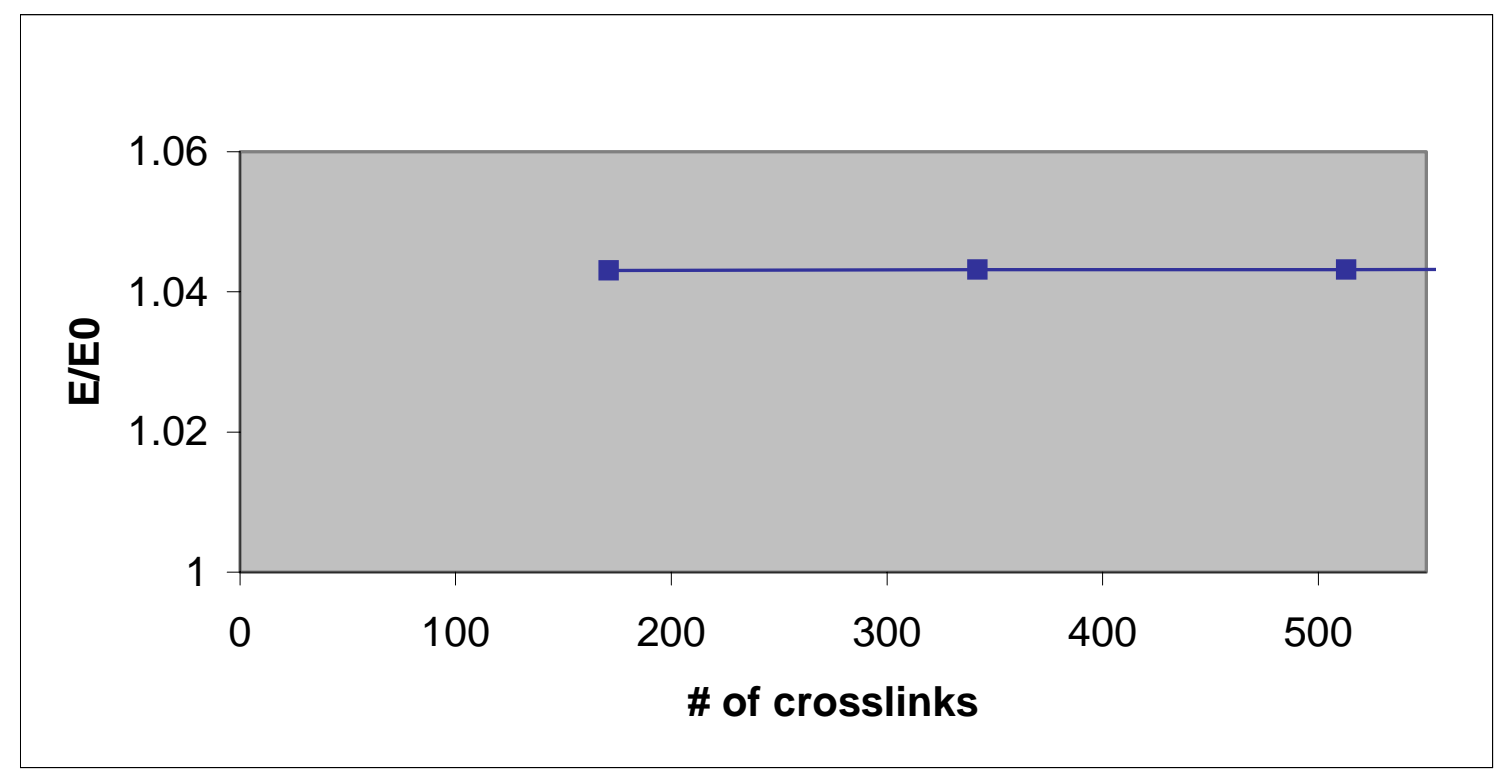

Figure 35 Variation of dimensionless equivalent young's modulus of the 3D cell model with number of crosslinks

A 3 fold increase in \# of crosslinks increased the equivalent young's modulus by less than $1 \%$ 


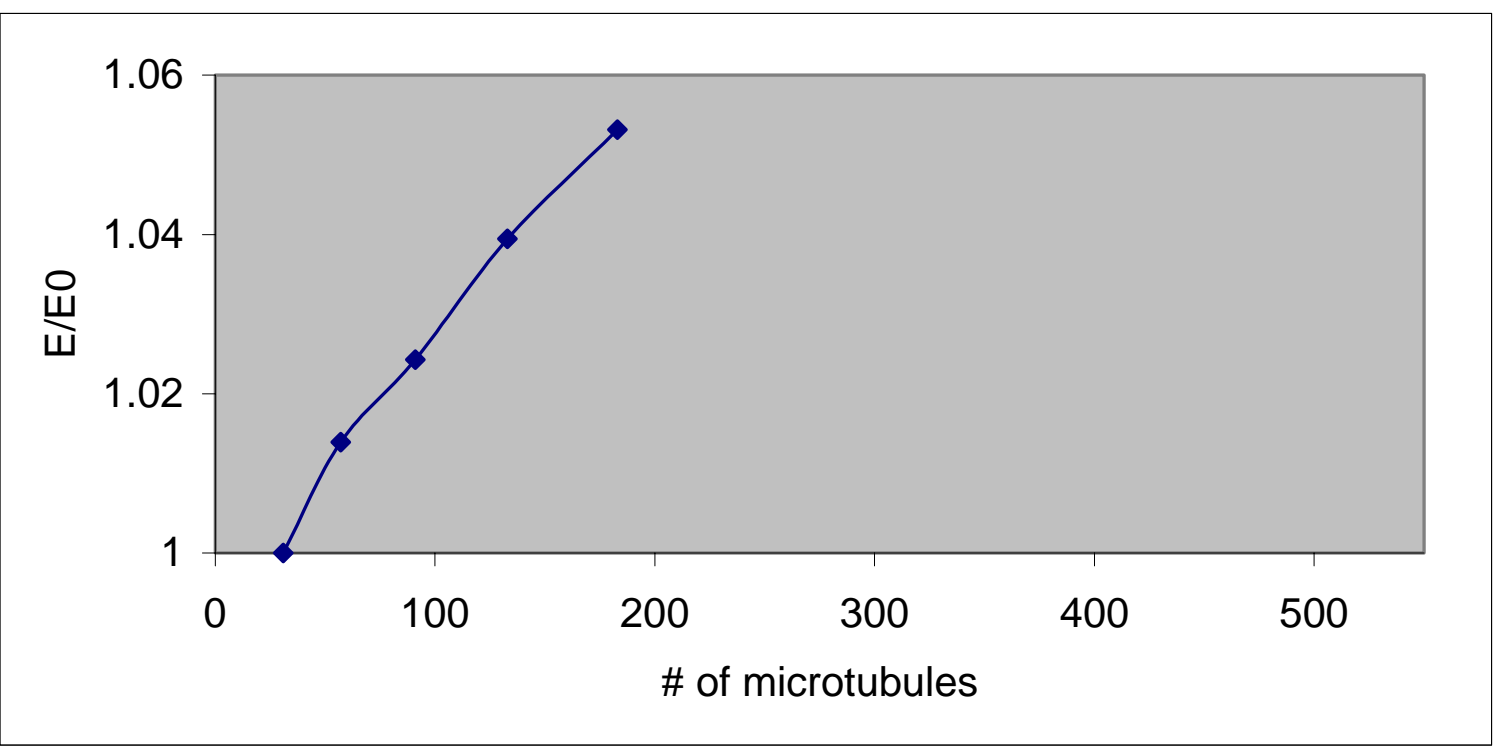

Figure 36 Variation of dimensionless equivalent young's modulus of 3D cell model with number of microtubules.

4 fold increase in number of microtubules increased the equivalent young's modulus by $6 \%$

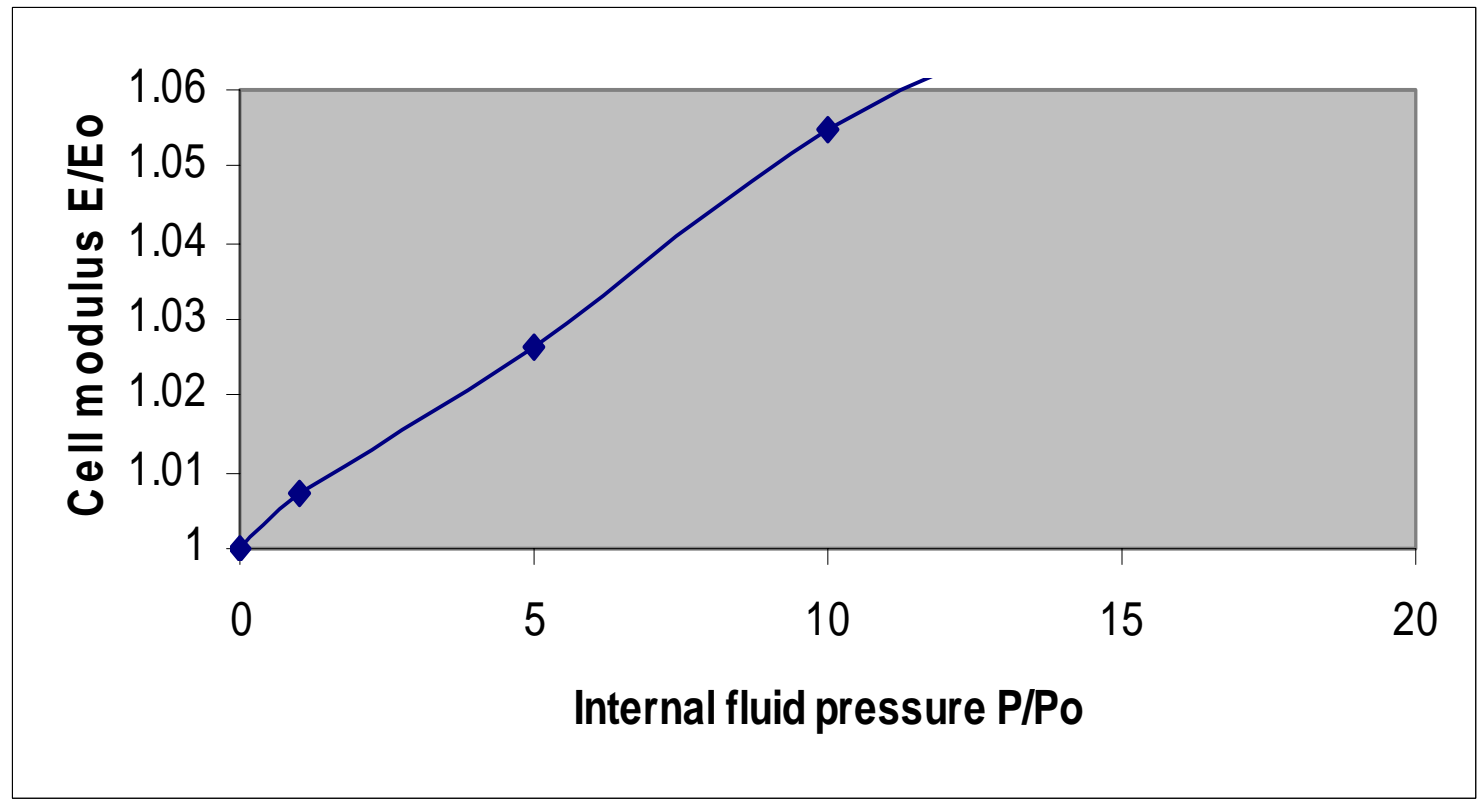

Figure 37 A variation of dimensionless young's modulus of cell (E/E0) with the variation of dimensionless internal fluid pressure (P/P0).

A 10 fold increase in internal Fluid pressure caused a $6 \%$ increase in equivalent young's modulus. 


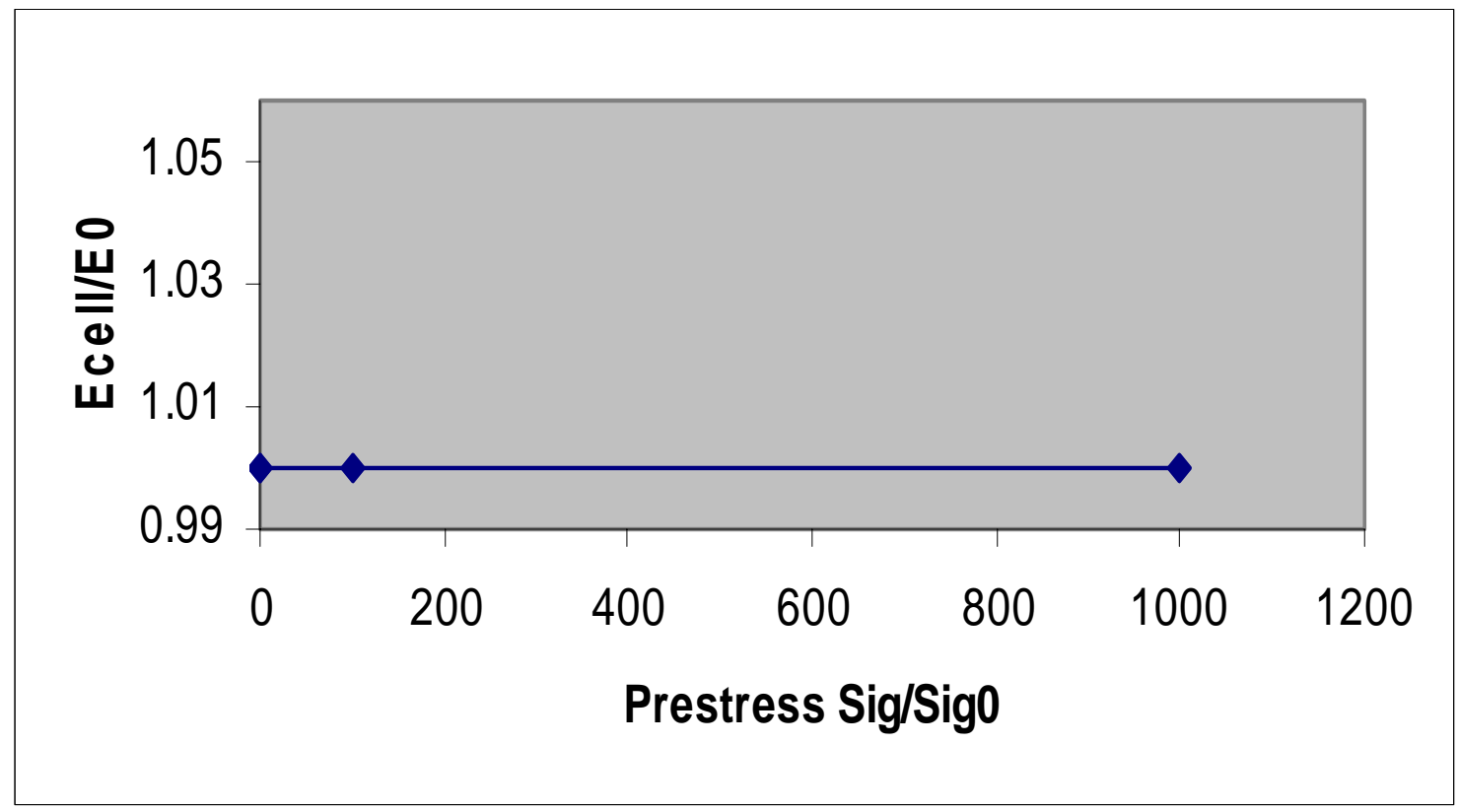

Figure 38 Variation of dimensionless young's modulus of cell (E/E0) with the variation of dimensionless prestress of the actin filaments (Sig/Sig0).

A 1000 increase in the pre-stress of the actin filaments increased the equivalent young's modulus by less than $0.01 \%$

\subsection{Discussion}

A uniaxial loading caused the cell to compress in the direction of the load and slightly elongate perpendicular to the direction of the load. The predicted radial deformations of an inclusion embedded in an infinite medium from Goodiers solutions showed a similar trend. For a given number of cytoskeletal elements, internal fluid pressure and actin pre-stress an equivalent young's modulus was found by fitting it with a closed form solution of spherical inclusion inside a medium subjected to compressive stress. The graphs show how the 
equivalent modulus of elasticity changed when the structural features of the cell were changed.

When the Pre-stress in microtubules were increased by 1000 fold the equivalent young's modulus increased by less than $0.01 \%$. A 4-fold increase in number of microtubules increased the equivalent young's modulus by $6 \%$. A 3 fold increase in \# of crosslinks increased the equivalent young's modulus by less than $1 \%$. A 10 -fold increase in internal Fluid pressure caused a $6 \%$ increase in equivalent young's modulus.

This study shows that the presence of microtubules and the internal fluid pressure affect the over all stiffness of cell more compared to the actin crosslinks and actin prestress. The reason behind such a behavior could be due to uniaxial loading mainly taken up by microtubule elements and intra-fluid pressure. Also from the material and dimensional properties, the microtubules are known to be 1000 times stiffer than actin. So intuitively it makes sense that the effect of microtubules on overall stiffness of cell compared to actin filaments. Further it would be interesting to investigate the effect of each structural component on the overall stiffness of cell, by subjecting it to different loading conditions that are commonly seen in real life environment. This includes different conditions like shear loading, local pulling stress etc., 


\section{Chapter 8: Conclusion and Future Studies}

\subsection{Discussion}

In this study we developed and validated a finite element model of a cell that incorporates the most important structural components of the cell - cell membrane, cytoskeletal structure and intracellular fluid. The cell was modeled as a $1 / 8^{\text {th }}$ of sphere consisting of cell membrane, microtubules and actin filaments forming an interconnected network and intracellular fluid applying pressure. The behavior of the model was predicted, when it was subjected to different kinds of loading conditions like compressive, uniaxial compressive, point-loads (pulling and compressive) etc.,

As a first step the cell model was validated with closed form solution of spherical inclusion inside a medium solved by Goodier., 1933. The closeness of the match between our cell model with the Goodier's model gave the confidence that the results obtained are believable. The effect of each structural component on the overall stiffness of the cell subjected to compressive loading was studied. The parametric study showed that internal fluid pressure has a bigger influence on the overall stiffness of the cell compared to the cytoskeletal elements. The reason behind such a behavior could be that the number of cytoskeletal elements is very less compared to that actually seen in a cell. The volume fraction of the cytoskeletal elements in a cell is very less. So their impact on the overall stiffness of the cell is less than that of internal fluid pressure.

Next the buckling behavior of the microtubules were studied. An eigen value buckling analysis in ABAQUS predicted the critical load for a microtubule column to buckle 
to be 23.57. This was validated with theoretical calculations. A riks analysis was used to study the post-buckling behavior of the microtubule column. The addition of actin filaments as lateral elements stabilized the microtubule column prone to buckling. This could be the reason that in a cell, the microtubule is prevented from buckling by presence of large number of actin cross-links. Also a small pre-stress in actin filaments caused a slight increase in stiffness to buckling as it stiffened the free edge of the microtubule but as when the pre-stress was further increased, it decreased the stiffness of microtubule. This was due to a compressive load being added due to the pre-stress in actin elements, increasing the microtubule's propensity to buckle.

Next the cell model was built in layers, starting with microtubule and actin filaments. When all the structural elements were added in layers, the stresses were distributed to all the structural elements. The distribution of stresses on the all the structural elements made sense intuitively.

Next the buckling behavior of microtubule in a 3D cell structure was studied. Due to presence more lateral constraints in the form of actin filaments, the microtubule is more stable to buckling. Also the behavior of buckling of microtubules, in the presence of actin pre-stress showed a similar trend as seen in flag model. When a small tensile pre-stress, was applied to actin filaments, it increased the post-buckling stability. But as the tensile pre-stress was increased, the deformations increased, indicating decreased post-buckling stability. Therefore pre-stress in actin plays a role in post buckling stability of microtubules in a cell. Also when a point pulling and point compressive loads was applied at the free edge of microtubule in a cell model, it caused the neighboring microtubules also to buckle. Also it was seen that the microtubules away from the point of application of the load also deformed 
and even buckled. This behavior is attributed to the action at a distance observed in a cell. So having verified the behavior of various structural elements of the cell, next step is to combine all the features to study the behavior of whole cell to different environmental conditions.

Simulating the environment of cells embedded in a gel subject to compressive loads, our 3D cell model was subjected to uniaxial compressive load applied to all nodes at the outer membrane. From the deformation data obtained for a cell model subjected to uniaxial compression, equivalent Young's modulus was calculated. This was done by fitting the deformation curve with that of closed form solution of spherical inclusion inside a infinite medium. Thus the overall stiffness of the cell model was measured in terms of its Equivalent Young's modulus. A parametric study on the effect of each structural component of cell on the overall stiffness of cell was performed. The study showed that the microtubules and internal fluid pressure played a major role on the overall stiffness of cell compared to the actin filaments and the pre-stress in actins. The reason for such a behavior could be due to, the kind of loading. The microtubules take up major part of the uniaxial loading and hence the effect of actin filaments on the overall stiffness of cell is less.

Thus such a model can be further used to study the behavior of the cell to different mechanical environments representing different experimental set up. The present model of cell is very stiff, even though the realistic material properties are used for the cell model. This shows that there is a need to give importance to viewing the cell as a structure rather than a purely continuum body. Also viewing in such a dimension would give a idea on how the cell responds to its mechanical environment and also how the biological factors change in response to the mechanical loads. 


\subsection{Limitations}

Thought the model can predict various cellular behavior observed experimentally, there are some limitations that are to over come for predicting the behavior more accurately. The actual values of the pre-stress in actin and microtubules are not known, and hence only a arbitrary pre-stress values were applied.

In our cell model the organization of the cytoskeletal elements are simple and neat. But the cytoekeletal elements are known to be organized in a more complex organization in a cell. This can be overcome by developing a finite element model of a cell from a realistic cytoskeletal structure of a cell.

The actual value of intracellular fluid pressure is not known. So an arbitrary value of internal fluid pressure is assumed. Also the cell membrane is assumed to be fully permeable and hence when compressed, the fluid from inside the cell freely flows out. But in reality the cell membrane is not fully permeable.

The microtubles and actin crossections are assumed to be perfectly circular, but the actual structure of microtubules and actins are much more complicated.

The juction where microtubules are attached to other actins and membranes are considered to be rigidly fixed, hence, though our model can predict the action at a distance phenomenon, the load required for seeing such an behavior is much higher than actually required. This can be overcome by using multiple point constraints where in the junction where the actin and microtubules are attached move together but are free to rotate with respect to each other. 


\subsection{Future studies}

The present model of cell is very stiff, even though the realistic material properties are used for the cell model. This shows less rigid constraints are needed to be applied at the juctions between the actin and microtubules. This can be achieved by using Multiple point constraints in ABAQUS.

A realistic cytoskeletal architecture can be modeled by reconstructing the images of the cytoskeletal structure to a finite element model. This would give a more accurate prediction of the behavior of cell to different mechanical environments. 


\section{References}

1. Buckwalter, J.A., T.A. Einhorn, and S.R. Simon, Orthopaedic Basic Science. 2nd Edition ed. 2000: American Academy of Orthopaedic Surgeons.

2. Hutchinson, T.M., et al., Factors in daily physical activity related to calcaneal mineral density in men. Med Sci Sports Exerc, 1995. 27(5): p. 745-50.

3. Goodship, A.E., et al., Bone loss during long term space flight is prevented by the application of a short term impulsive mechanical stimulus. Acta Astronaut, 1998. 43(3-6): p. 65-75.

4. Schneider, V., et al., Bone and body mass changes during space flight. Acta Astronaut, 1995. 36(8-12): p. 463-6.

5. Wittich, A., et al., Body composition of professional football (soccer) players determined by dual X-ray absorptiometry. J Clin Densitom, 2001. 4(1): p. 51-5.

6. Andreoli, A., et al., Effects of different sports on bone density and muscle mass in highly trained athletes. Med Sci Sports Exerc, 2001. 33(4): p. 507-11.

7. Buschmann, M.D., et al., Mechanical compression modulates matrix biosynthesis in chondrocyte/agarose culture. J Cell Sci, 1995. 108(Pt 4): p. 1497-508.

8. Guilak, F. and V.C. Mow, The mechanical environment of the chondrocyte: a biphasic finite element model of cell-matrix interactions in articular cartilage. $\mathrm{J}$ Biomech, 2000. 33(12): p. 1663-73.

9. Durrant A., et al., Organisation of the chondrocyte cytoskeleton and its response to changing mechanical condtions in organ culture. J. Anat., 1999. 194: p. 343-353.

10. Guilak, F., Compression-induced changes in the shape and volume of the chondrocyte nucleus. J Biomech, 1995. 28(12): p. 1529-41. 
11. Guilak, F., The deformation behavior and viscoelastic properties of chondrocytes in articular cartilage. Biorheology, 2000. 37(1-2): p. 27-44.

12. Guilak, F., et al., The deformation behavior and mechanical properties of chondrocytes in articular cartilage. Osteoarthritis Cartilage, 1999. 7(1): p. 59-70.

13. Shin, D. and K. Athanasiou, Cytoindentation for obtaining cell biomechanical properties. J Orthop Res, 1999. 17(6): p. 880-90.

14. Freeman, P.M., et al., Chondrocyte cells respond mechanically to compressive loads. J Orthop Res, 1994. 12(3): p. 311-20.

15. Somjen D, Binderman I, and Berger E, Bone remodeling induced by physical stress is prostagladin E2 mediated. Biochim Biophys Acta, 1980. 627: p. 91-100.

16. Buschmann MD, et al., Altered aggrecan synthesis correlates with cell and nucleus structure in statically compressed cartilage. journal of Cell Science, 1996. 109: p. 499-508.

17. Thomas Quinn M, et al., Mechanical compression alters proteoglycan deposition and matrix deformation around individual cells in cartilage explants. journal of Cell Science, 1998. 111: p. 573-583.

18. Robert L Mauck, et al., Functional tissue engineering of articular cartilage through dynamic loading of chondrocyte-seeded agarose gels. journal of biomechanical engineering, 2000. 122: p. 252-260.

19. Hochmuth, R.M. and N. Mohandas, Uniaxial loading of the red-cell membrane. J Biomech, 1972. 5(5): p. 501-9.

20. Alberts, B., et al., Molecular biology of the cell. 3rd ed. 1994, New York and London: Garland Publishing, Inc. 
21. Bruce Alberts, D.B., Julian Lewis, Martin Raff, Keith Roberts, James D. Watson, Molecular Biology of THE CELL. 3 ed. 1994.

22. Gittes, F., et al., Flexural rigidity of microtubules and actin filaments measured from thermal fluctuations in shape. J Cell Biol, 1993. 120(4): p. 923-34.

23. Smith, A.E., On uniquely determining cell-wall material properties with the compression experiment. Chem Eng Sci, 1998. 53(23): p. 3913-3922.

24. Smith, A.E., Wall material properties of yeast cells: Part I. Cell measurements and compression experiments. Chem Eng Sci, 2000. 55: p. 2031-2041.

25. Durrant, L.A., et al., Organisation of the chondrocyte cytoskeleton and its response to changing mechanical conditions in organ culture. J Anat, 1999. 194(Pt 3): p. 343-53.

26. Benya, P.D. and J.D. Shaffer, Dedifferentiated chondrocytes reexpress the differentiated collagen phenotype when cultured in agarose gels. Cell, 1982. 30(1): p. 215-24.

27. Zanetti, N.C. and M. Solursh, Induction of chondrogenesis in limb mesenchymal cultures by disruption of the actin cytoskeleton. J Cell Biol, 1984. 99(1 Pt 1): p. 11523.

28. Parker, K.K., et al., Directional control of lamellipodia extension by constraining cell shape and orienting cell tractional forces. Faseb J, 2002. 16(10): p. 1195-204.

29. Gopalan, S.M., et al., Anisotropic stretch-induced hypertrophy in neonatal ventricular myocytes micropatterned on deformable elastomers. Biotechnol Bioeng, 2003. 81(5): p. 578-87.

30. Wang, N., et al., Mechanical behavior in living cells consistent with the tensegrity model. Proc Natl Acad Sci U S A, 2001. 98(14): p. 7765-70. 
31. Jones, W.R., et al., Alterations in the Young's modulus and volumetric properties of chondrocytes isolated from normal and osteoarthritic human cartilage [see comments]. J Biomech, 1999. 32(2): p. 119-27.

32. Ingber, D.E., Opposing views on tensegrity as a structural framework for understanding cell mechanics. J Appl Physiol, 2000. 89(4): p. 1663-70.

33. Stamenovic, D., et al., Cell prestress. II. Contribution of microtubules. Am J Physiol Cell Physiol, 2002. 282(3): p. C617-24.

34. Dennerll, T.J., et al., Tension and compression in the cytoskeleton of PC-12 neurites. II: Quantitative measurements. J Cell Biol, 1988. 107(2): p. 665-74.

35. Wang, N., et al., Cell prestress. I. Stiffness and prestress are closely associated in adherent contractile cells. Am J Physiol Cell Physiol, 2002. 282(3): p. C606-16.

36. Smith, A.E., et al., The mechanical properties of Saccharomyces cerevisiae. Proc Natl Acad Sci U S A, 2000. 97(18): p. 9871-4.

37. Smith, A.E., Wall material properties of yeast cells. Part II. Analysis. Chem Eng Sci, 2000. 55: p. 2043-2053.

38. Strohmeier, R. and J. Bereiter-Hahn, Hydrostatic pressure in epidermal cells is dependent on Ca-mediated contractions. J Cell Sci, 1987. 88(Pt 5): p. 631-40.

39. Goodier, J.N., Concentration of stress around spherical and cylindrical inclusions and flows. J Applied Mechanics, 1933. 55: p. 39-44.

40. Ingber, D.E., Cellular tensegrity: defining new rules of biological design that govern the cytoskeleton. journal of Cell Science, 1993. 104: p. 613-627.

41. Ingber, D.E., Cellular tensegrity: defining new rules of biological design that govern the cytoskeleton. J Cell Sci, 1993. 104(Pt 3): p. 613-27. 
42. Volokh, K.Y., O. Vilnay, and M. Belsky, Tensegrity architecture explains linear stiffening and predicts softening of living cells. J Biomech, 2000. 33(12): p. 1543-9.

43. M.F Coughlin, D.S., A Tensigrity Structure With Buckling Compression Elements: Application to Cell Mechanics. Journal Of Applied Mechanics, 1997. 64: p. 480-486.

44. Ferdinand P.Beer, E.R.J., Jr, John T. Dewolf, Mechanics Of Materials. third ed. 2000. 


\section{Appendix 1}

\section{Program for generation of 3D finite Element model of a cell}

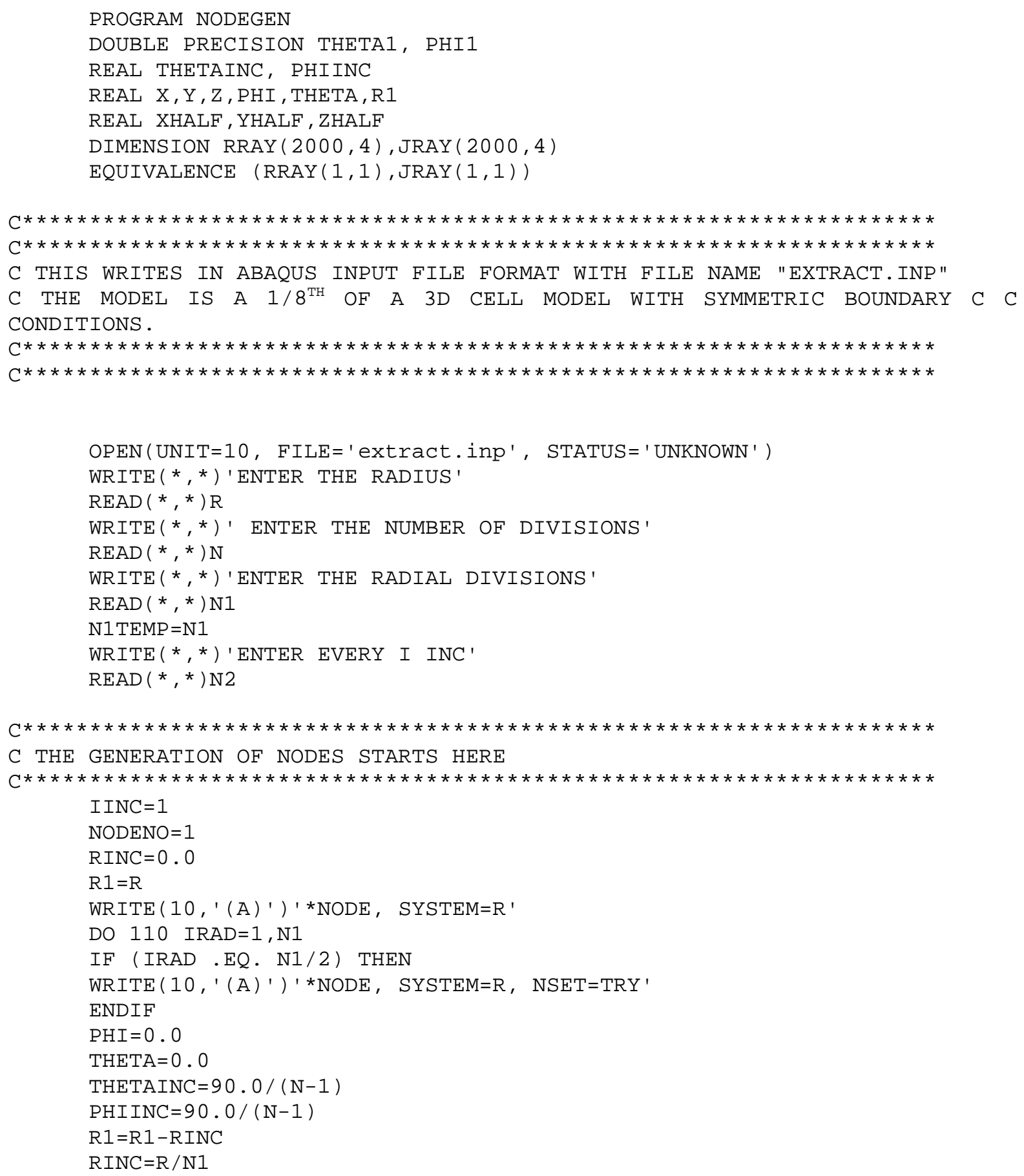


$\operatorname{WRITE}(10, *) \mathrm{R} 1$

DO 100 IVERTNO $=1, \mathrm{~N}-1$

DO 200 IHORINO $=1, \mathrm{~N}$

THETA $1=\operatorname{THETA} *(22.0 /(7.0 * 180.0))$

PHII $=$ PHI* $(22.0 /(7.0 * 180.0))$

$\mathrm{X}=\left(\left(\mathrm{R} 1{ }^{*} \operatorname{COS}(\mathrm{THETA} 1)\right) \star \operatorname{COS}(\mathrm{PHI} 1)\right)$

IF (X .LT. 0.0) THEN

$\mathrm{X}=0.0$

ENDIF

$\mathrm{Y}=(\mathrm{R} 1 * \operatorname{SIN}(\mathrm{PHI} 1))$

$\mathrm{Z}=\left(\left(\mathrm{R} 1{ }^{*} \operatorname{COS}(\mathrm{PHI} 1)\right) * \operatorname{SIN}(\mathrm{THETA} 1)\right)$

WRITE $(10,1000)$ NODENO, X, Y, Z

IF (IRAD . EQ. ( (N1/2)+1)) THEN

$R P=S Q R T((X \star X)+(Y \star Y)+(Z \star Z))$

IF (Z .NE. 0.0) THEN

$\mathrm{RZCOORD}=\mathrm{RP} * \mathrm{RP} / \mathrm{Z}$

RZDASH $=(Z-R Z C O O R D)$

IF (RZDASH .NE. 0.0) THEN

$\operatorname{RPDASH}=\operatorname{SQRT}((\mathrm{X} * \mathrm{X})+(\mathrm{Y} * \mathrm{Y})+(\mathrm{RZDASH} * \mathrm{RZDASH}))$

WRITE $(*, *)$ NODENO, $X, Y, Z, R Z D A S H, R P D A S H$

WRITE $(*, *)$ RZDASH /RPDASH

JRAY $($ IINC, 1$)=$ NODENO

RRAY $(I I N C, 2)=X / R P D A S H$

RRAY (IINC, 3) =Y / RPDASH

RRAY (IINC, 4$)=$ RZDASH $/$ RPDASH

$\operatorname{WRITE}(*, *)$ NODENO, X, Y, Z

$\operatorname{WRITE}(*, *) \operatorname{JRAY}(\operatorname{IINC}, 1), \operatorname{RRAY}(\operatorname{IINC}, 2)$, RRAY (IINC, 3)

$I I N C=I I N C+1$

ENDIF

ENDIF

ENDIF

NODENO $=$ NODENO +1

THETA $=$ THETA+THETAINC

$200 \quad$ CONTINUE

$\mathrm{PHI}=\mathrm{PHI}+\mathrm{PHIINC}$

$\mathrm{THETA}=0.0$

$100 \quad$ CONTINUE

WRITE $(10,1000)$ NODENO, 0, R1, 0

IF (IRAD .EQ. ( (N1/2)+1)) THEN

WRITE $(*, *)$ NODENO, $0, \mathrm{R} 1,0$

ENDIF

$\mathrm{NODENO}=\mathrm{NODENO}+1$

$110 \quad$ CONTINUE

WRITE $(10,1000) 10000,0,0,0$

1000 FORMAT (I, ', ', E14.8, ' ' ', E14.8, ' , ', E14.8)

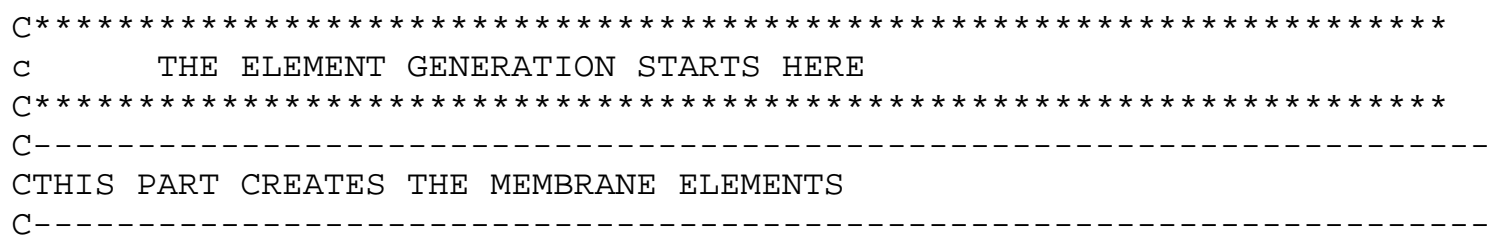

IELNO $=1$

$\mathrm{I}$ TEMP $=0$

$\mathrm{LEVEL} 1=0$ 


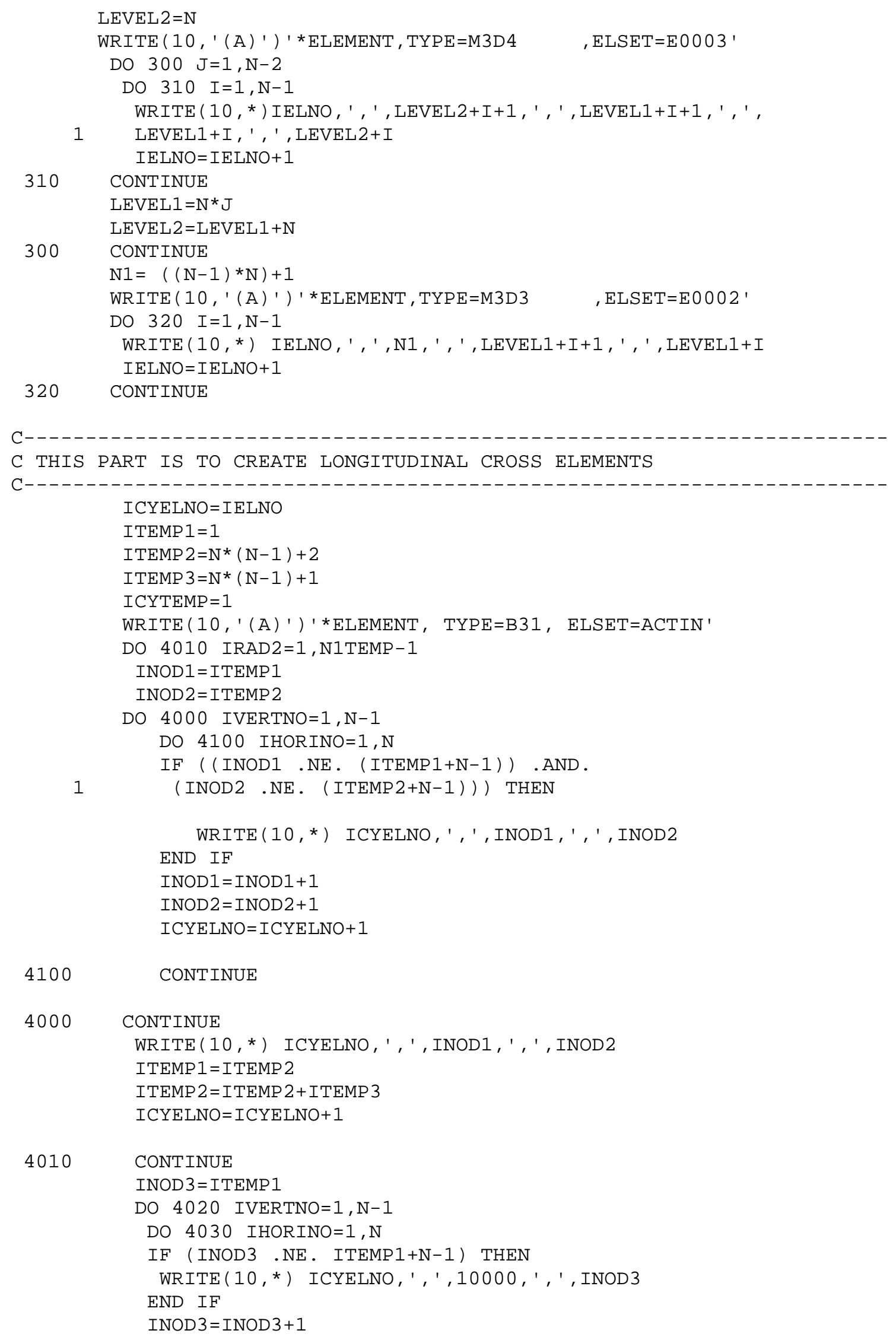




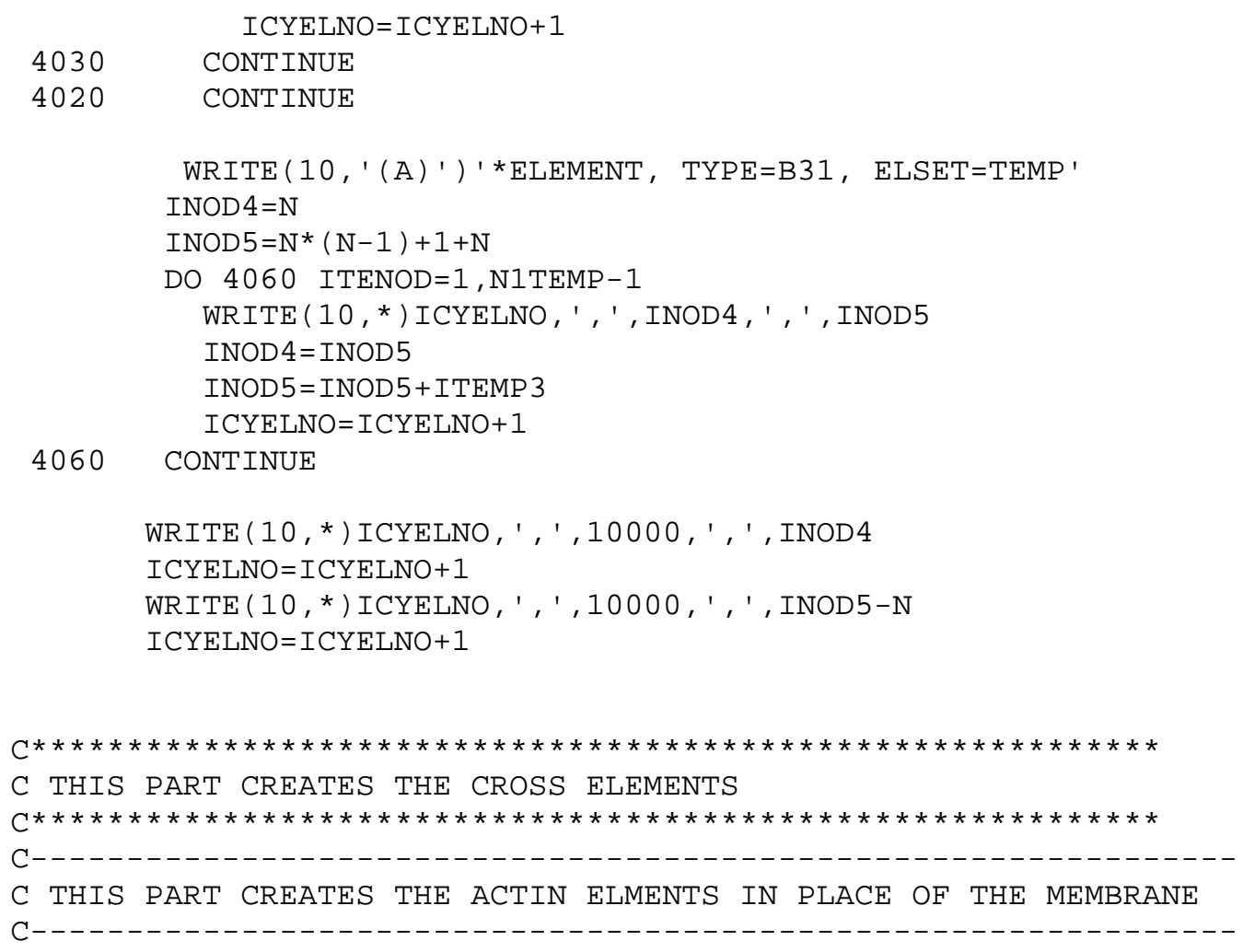




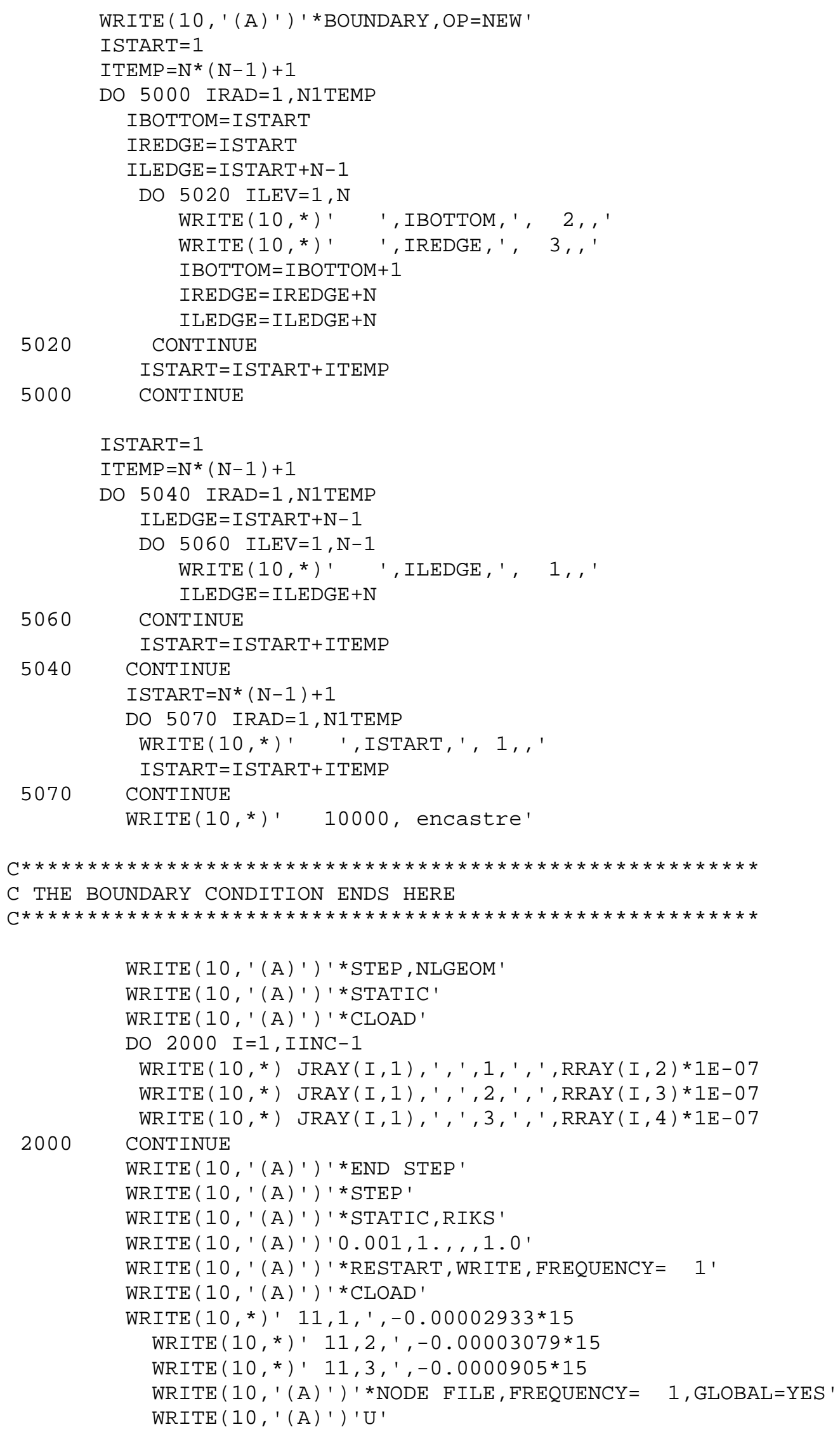


WRITE $(10, '$ (A) ') '*EL FILE, FREQUENCY= 1, POSITION=NODES ' WRITE $(10, '(A)$ ') 'S'

WRITE $(10, '$ (A) ' ) ' NODE PRINT, FREQUENCY $=1$, GLOBAL=YES ' WRITE $(10, '(A)$ ' ) 'U' WRITE $(10, '(A) ') ' \star E N D$ STEP' END 


\section{Appendix 2}

\section{$\underline{\text { Nodes generation model-calculation }}$}

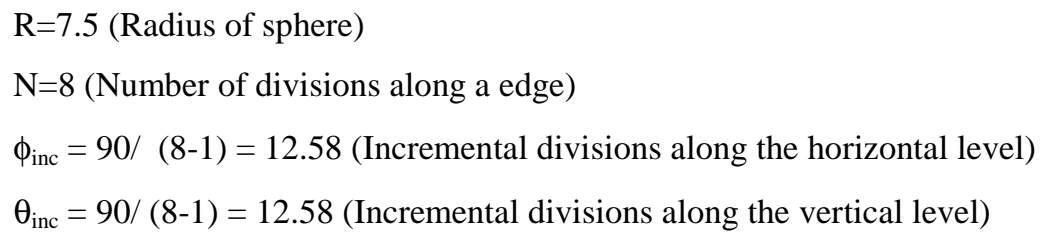

For :

$$
\begin{aligned}
& \theta=12.58 \\
& \phi=12.58
\end{aligned}
$$

$\mathrm{X}$-coord $=\mathrm{R} * \cos (\theta) * \cos (\phi)$

$$
=7.5 * \cos (12.85) * \cos (12.85)
$$

$\underline{X-\text { coord }=7.129}$

$$
\begin{aligned}
\mathrm{Y} \text {-coord } & =\mathrm{R} * \sin (\phi) \\
& =7.5 * \sin (12.85) \\
\underline{\mathrm{Y} \text {-coord }} & =1.6679 \\
\mathrm{Z} \text {-coord } & =\mathrm{R} * \cos (\phi) * \sin (\theta) \\
& =7.5 * 0.9762 * 0.2223 \\
\underline{\mathrm{Z}-\operatorname{coord}} & =1.6277
\end{aligned}
$$




\title{
Appendix 3
}

\section{ABAQUS INPUT FILE REPRESENTING 3D CELL MODEL}

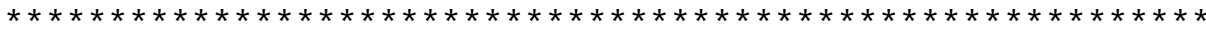

$\star \star$ CREATE NODES WITH NODE NUMBER AND THERE COORDINATES

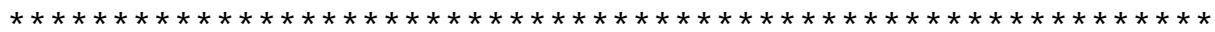

*NODE, SYSTEM=R

$1,0.75000000 \mathrm{E}+01,0.00000000 \mathrm{E}+00,0.00000000 \mathrm{E}+00$

$2,0.53016238 \mathrm{E}+01,0.00000000 \mathrm{E}+00,0.53049774 \mathrm{E}+01$

$3,0.00000000 \mathrm{E}+00,0.00000000 \mathrm{E}+00,0.74999986 \mathrm{E}+01$

$4,0.53016238 E+01,0.53049774 E+01,0.00000000 E+00$

$5,0.37476287 \mathrm{E}+01,0.53049774 \mathrm{E}+01,0.37499993 \mathrm{E}+01$

$6,0.00000000 \mathrm{E}+00,0.53049774 \mathrm{E}+01,0.53016229 \mathrm{E}+01$

$7,0.00000000 \mathrm{E}+00,0.75000000 \mathrm{E}+01,0.00000000 \mathrm{E}+00$

$8,0.60000000 \mathrm{E}+01,0.00000000 \mathrm{E}+00,0.00000000 \mathrm{E}+00$

$9,0.42412992 \mathrm{E}+01,0.00000000 \mathrm{E}+00,0.42439818 \mathrm{E}+01$

$10,0.00000000 \mathrm{E}+00,0.00000000 \mathrm{E}+00,0.59999986 \mathrm{E}+01$

$11,0.42412992 \mathrm{E}+01,0.42439818 \mathrm{E}+01,0.00000000 \mathrm{E}+00$

$12,0.29981029 \mathrm{E}+01,0.42439818 \mathrm{E}+01,0.29999993 \mathrm{E}+01$

$13,0.00000000 \mathrm{E}+00,0.42439818 \mathrm{E}+01,0.42412982 \mathrm{E}+01$

$14,0.00000000 \mathrm{E}+00,0.60000000 \mathrm{E}+01,0.00000000 \mathrm{E}+00$

$15,0.45000000 \mathrm{E}+01,0.00000000 \mathrm{E}+00,0.00000000 \mathrm{E}+00$

$16,0.31809742 \mathrm{E}+01,0.00000000 \mathrm{E}+00,0.31829865 \mathrm{E}+01$

$17,0.00000000 \mathrm{E}+00,0.00000000 \mathrm{E}+00,0.44999990 \mathrm{E}+01$

$18,0.31809742 \mathrm{E}+01,0.31829865 \mathrm{E}+01,0.00000000 \mathrm{E}+00$

$19,0.22485774 \mathrm{E}+01,0.31829865 \mathrm{E}+01,0.22499995 \mathrm{E}+01$

$20,0.00000000 \mathrm{E}+00,0.31829865 \mathrm{E}+01,0.31809738 \mathrm{E}+01$

$21,0.00000000 \mathrm{E}+00,0.45000000 \mathrm{E}+01,0.00000000 \mathrm{E}+00$

$22,0.30000000 \mathrm{E}+01,0.00000000 \mathrm{E}+00,0.00000000 \mathrm{E}+00$

$23,0.21206496 \mathrm{E}+01,0.00000000 \mathrm{E}+00,0.21219909 \mathrm{E}+01$

$24,0.00000000 \mathrm{E}+00,0.00000000 \mathrm{E}+00,0.29999993 \mathrm{E}+01$

$25,0.21206496 \mathrm{E}+01,0.21219909 \mathrm{E}+01,0.00000000 \mathrm{E}+00$

$26,0.14990515 \mathrm{E}+01,0.21219909 \mathrm{E}+01,0.14999996 \mathrm{E}+01$

$27,0.00000000 \mathrm{E}+00,0.21219909 \mathrm{E}+01,0.21206491 \mathrm{E}+01$

$28,0.00000000 \mathrm{E}+00,0.30000000 \mathrm{E}+01,0.00000000 \mathrm{E}+00$

$29,0.15000000 \mathrm{E}+01,0.00000000 \mathrm{E}+00,0.00000000 \mathrm{E}+00$

$30,0.10603248 \mathrm{E}+01,0.00000000 \mathrm{E}+00,0.10609955 \mathrm{E}+01$

$31,0.00000000 \mathrm{E}+00,0.00000000 \mathrm{E}+00,0.14999996 \mathrm{E}+01$

$32,0.10603248 \mathrm{E}+01,0.10609955 \mathrm{E}+01,0.00000000 \mathrm{E}+00$

$33,0.74952573 \mathrm{E}+00,0.10609955 \mathrm{E}+01,0.74999982 \mathrm{E}+00$

$34,0.00000000 \mathrm{E}+00,0.10609955 \mathrm{E}+01,0.10603245 \mathrm{E}+01$

$35,0.00000000 \mathrm{E}+00,0.15000000 \mathrm{E}+01,0.00000000 \mathrm{E}+00$

$10000,0.00000000 \mathrm{E}+00,0.00000000 \mathrm{E}+00,0.00000000 \mathrm{E}+00$
\end{abstract}




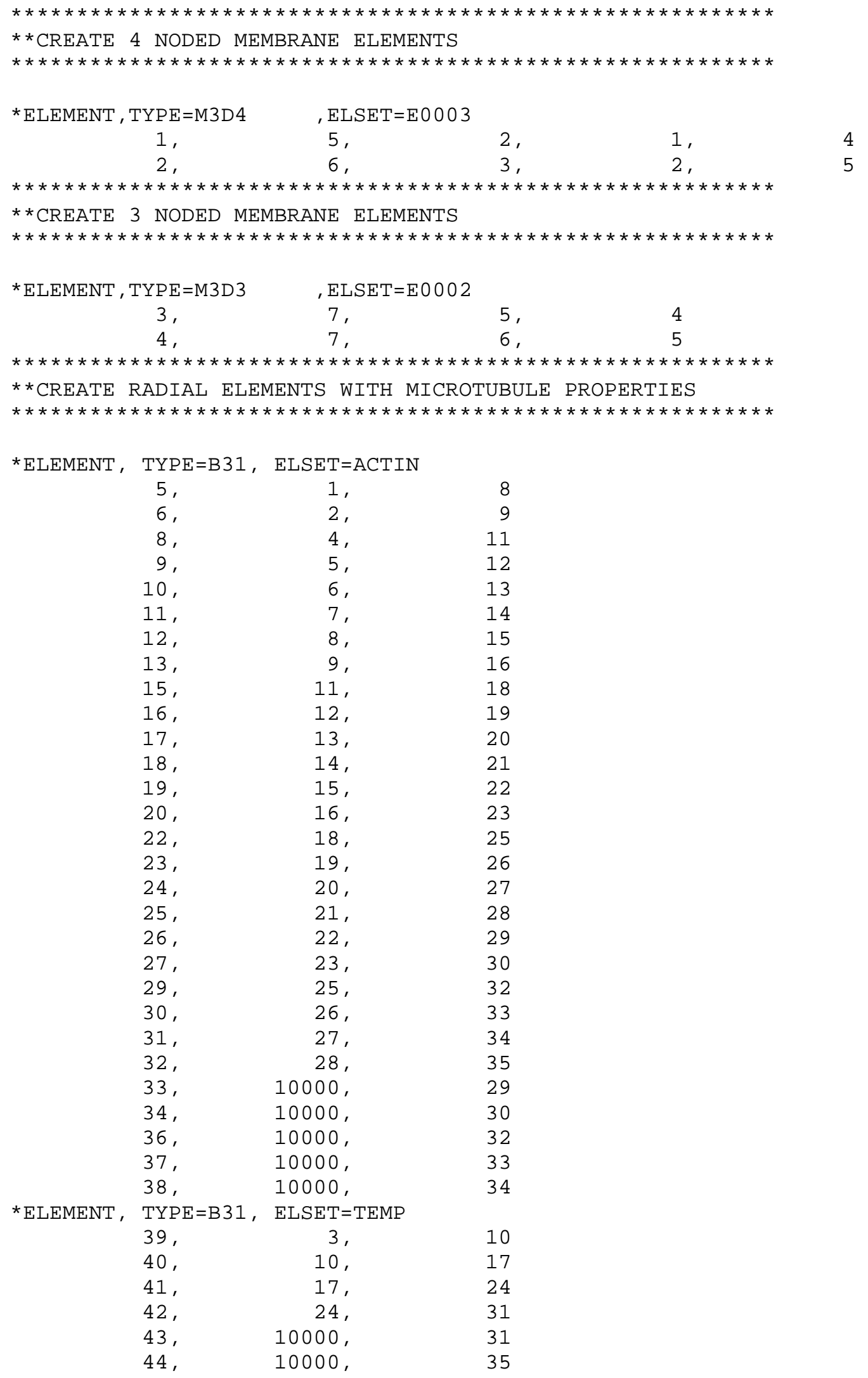




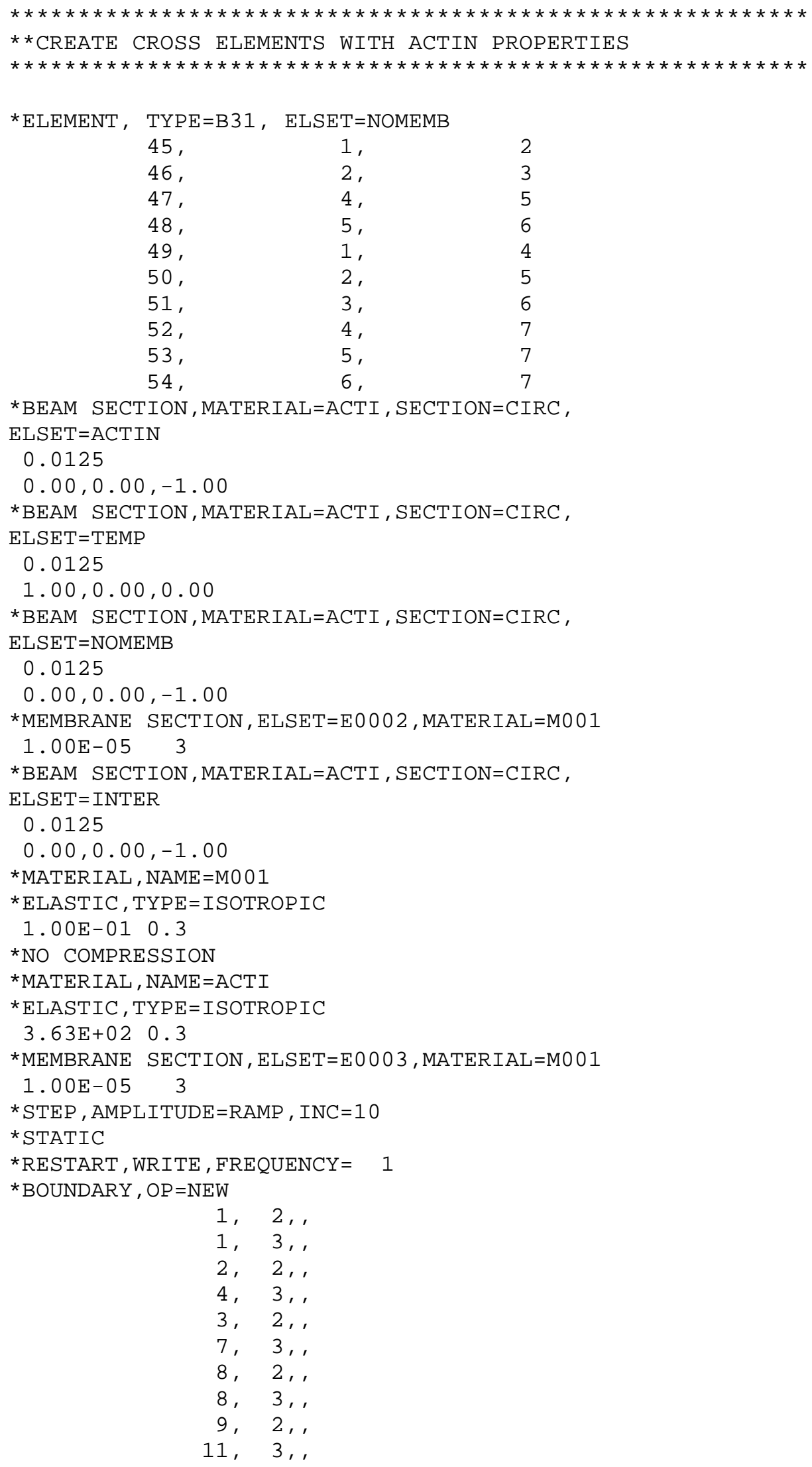




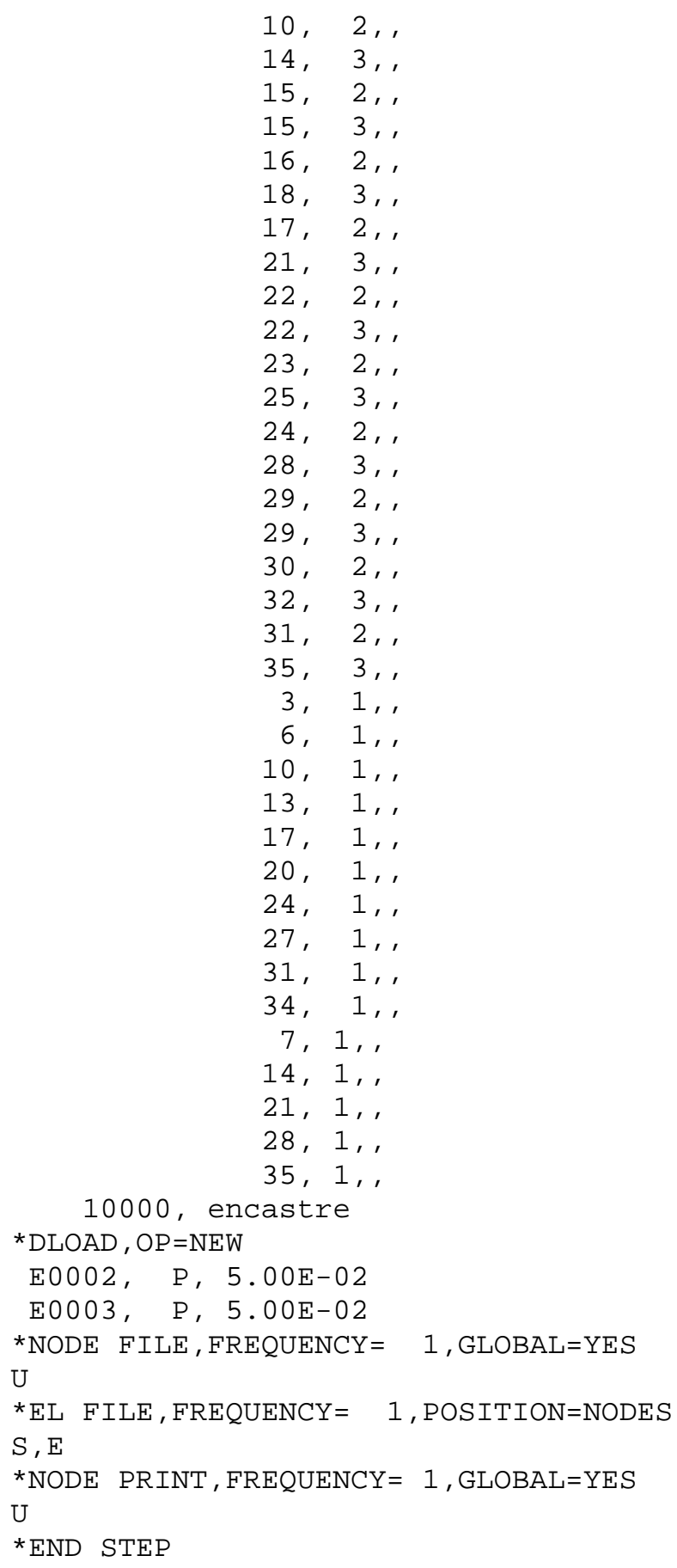




\section{Appendix 4}

Maple program for finding stress distribution around a spherical inclusion inside a medium.

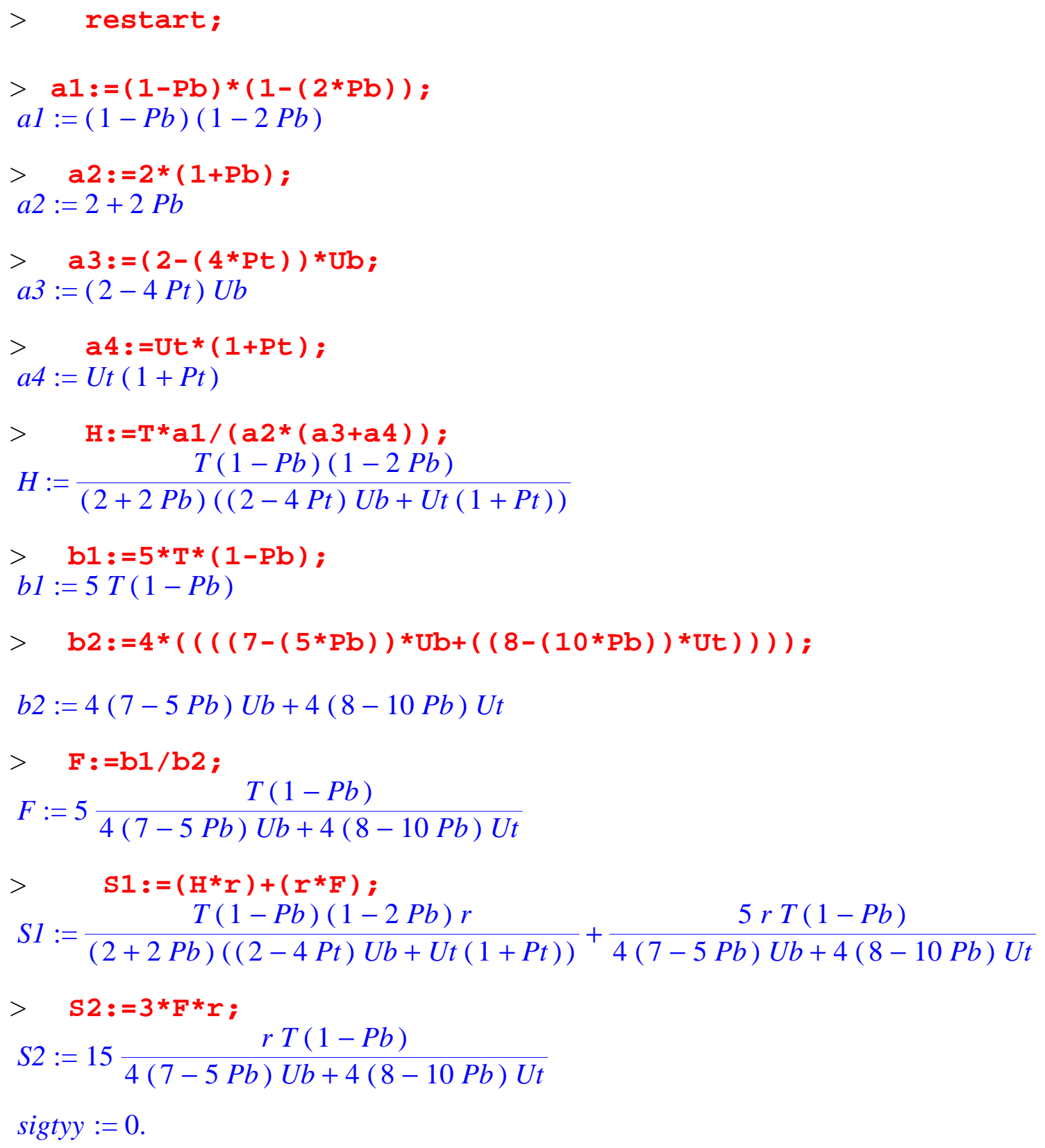




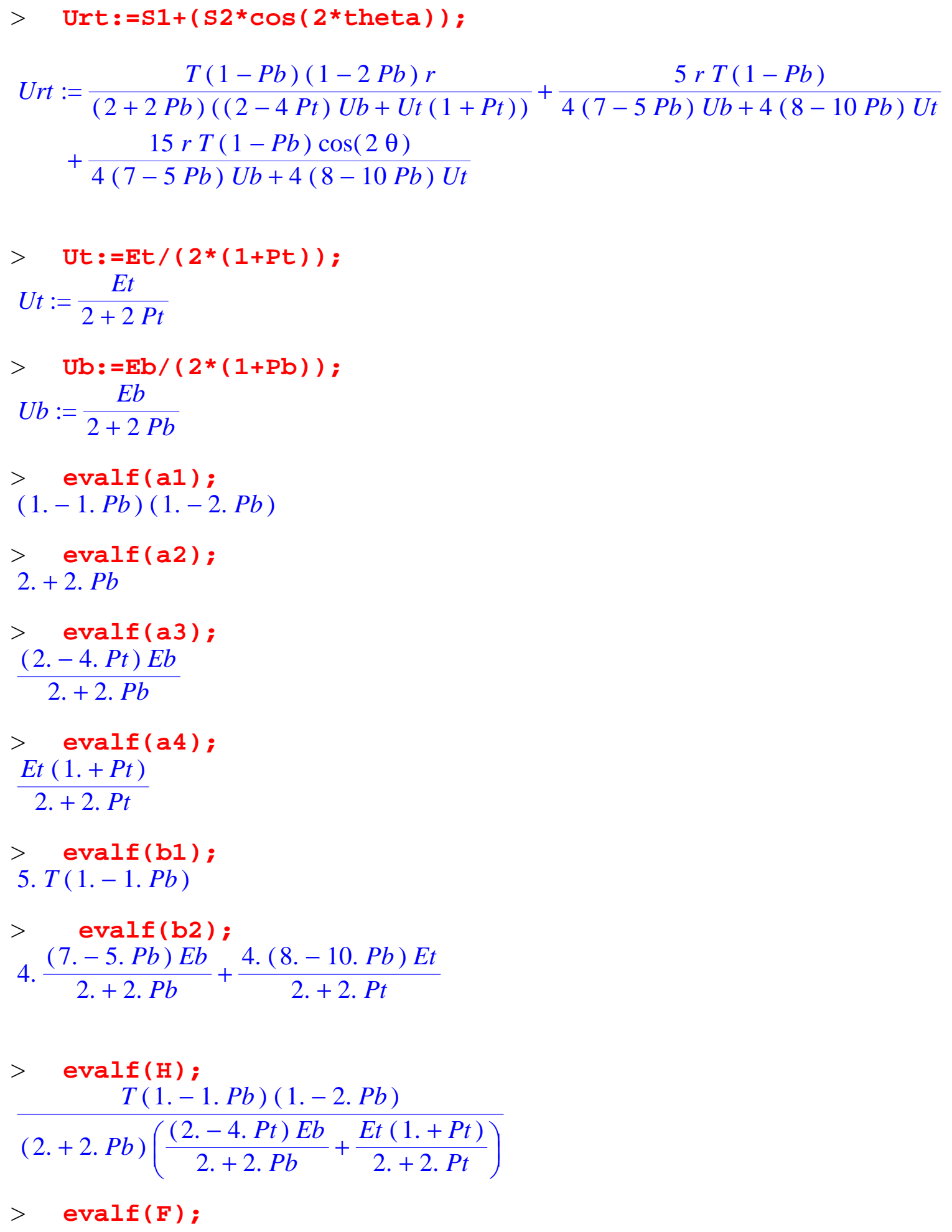




$$
\begin{aligned}
& \text { 5. } \frac{T(1 .-1 . P b)}{4 . \frac{(7 .-5 . P b) E b}{2 .+2 . P b}+\frac{4 .(8 .-10 . P b) E t}{2 .+2 . P t}} \\
& >.6666666665 \mathrm{e}-1 * \mathrm{~T} ; \\
& .06666666665 \mathrm{~T}
\end{aligned}
$$




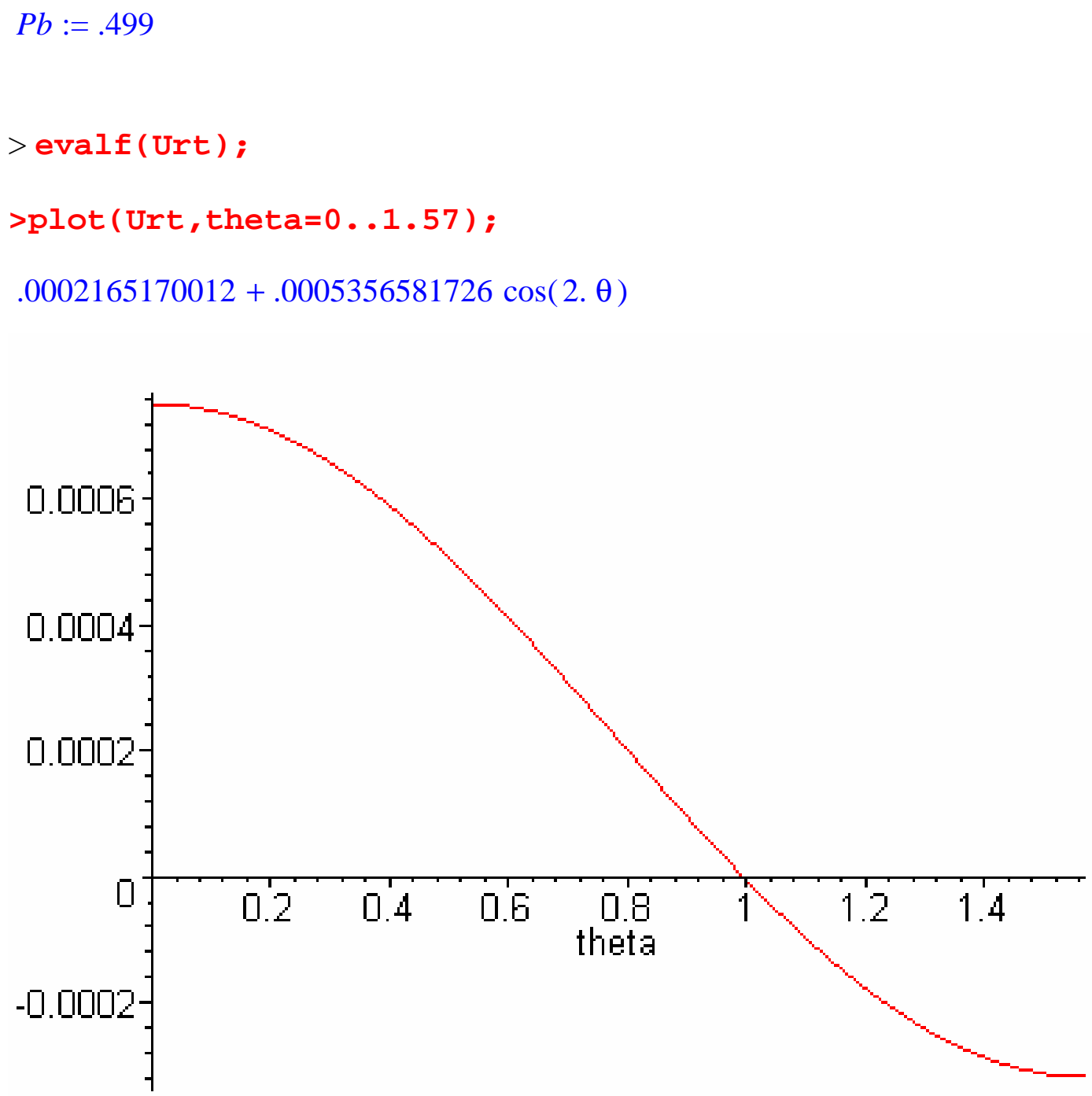




\section{Appendix 5}

\section{Representative ABAQUS input file for performing Buckling Analysis using RIKS-procedure}

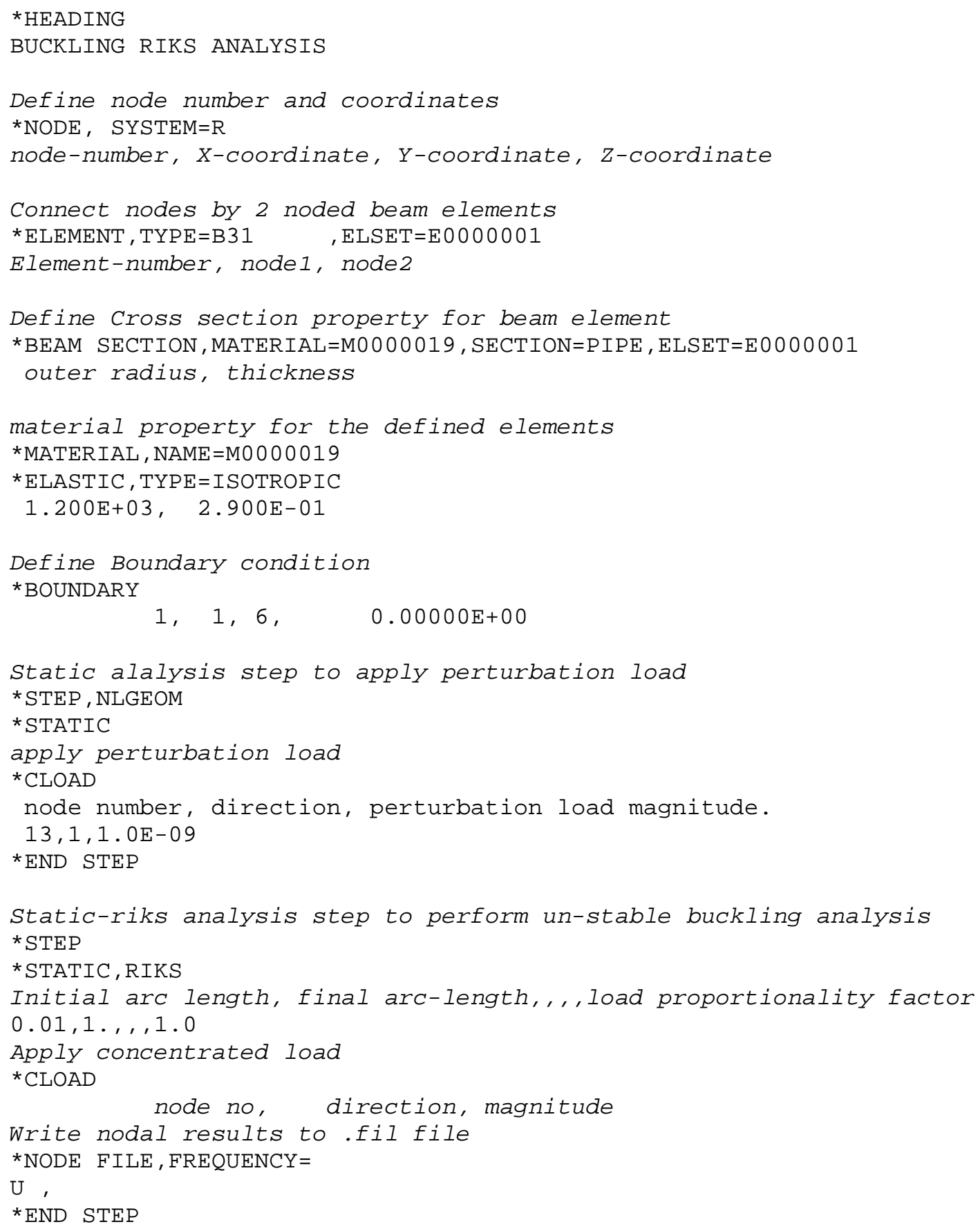




\section{Appendix 6}

\section{Resolving force along a line into there respective coordinates using Direction Cosines}

A line $(\mathrm{OP})$ in 3D space that joins 2 points $(0,0,0)$ and $(\mathrm{x}, \mathrm{y}, \mathrm{z})$ makes an angle of $\alpha, \beta$ and $\gamma$ with respect to the $\mathrm{x}$-axis, $\mathrm{y}$-axis and $\mathrm{z}$-axis as shown in fig \#\#

A force ' $\mathrm{F}$ ' is applied in the direction of the line OP. The force $\mathrm{F}$ can be resolved into there respective $\mathrm{x}, \mathrm{y}$ and $\mathrm{z}$ components. For finding the components of the force ' $\mathrm{F}$ ' direction cosines of the line OP is to be found.

To find the Direction cosine $(1, \mathrm{~m}, \mathrm{n})$ for the line OP that joins the points $(0,0,0)$ and $(\mathrm{x}, \mathrm{y}, \mathrm{z})$ following steps are followed.

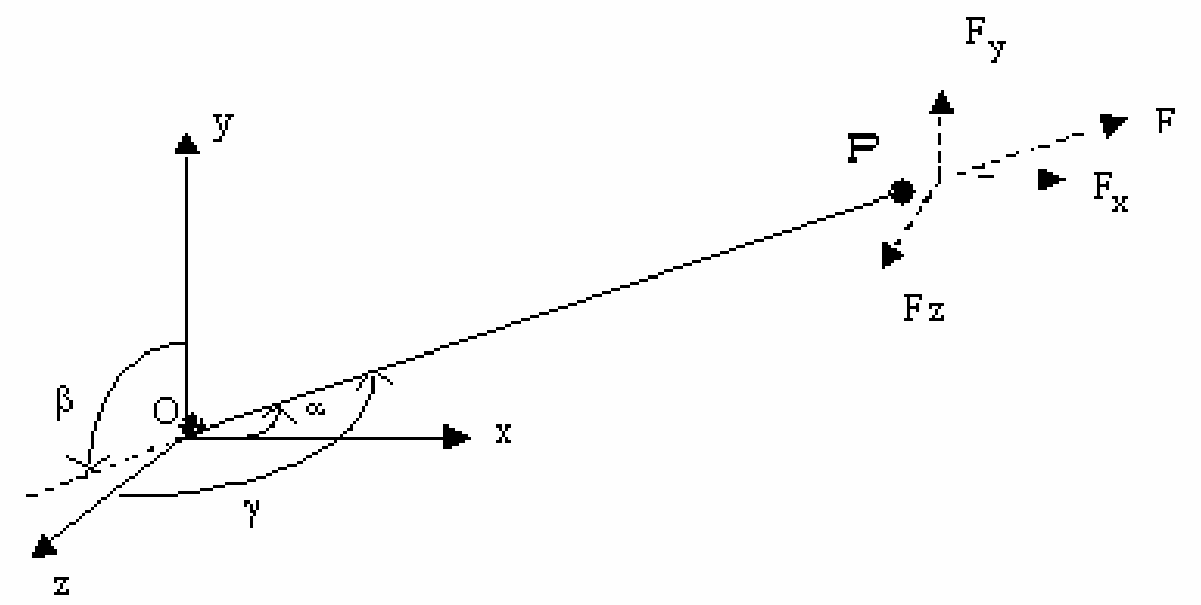

1.

Find

$$
r=\sqrt{x^{2}+y^{2}+z^{2}}
$$

2. The direction cosines are

$$
\begin{aligned}
& 1=\cos (\alpha)=x / r \\
& m=\cos (\beta)=y / r \\
& n=\cos (\gamma)=z / r
\end{aligned}
$$


There fore the force ' $F$ ' applied in the direction along the line OP can be resolved into its components

$$
\begin{aligned}
& \mathbf{F}_{\mathbf{z}}=(\mathbf{x} / \mathbf{r}) * \mathbf{F} \\
& \mathbf{F}_{\mathbf{y}}=(\mathbf{y} / \mathbf{r}) * \mathbf{F} \\
& \mathbf{F}_{\mathbf{z}}=(\mathbf{z} / \mathbf{r}) * \mathbf{F}
\end{aligned}
$$

\section{Force applied in a direction perpendicular to the line (OP)}

A line in 3D space OP as already described makes and angle $\alpha, \beta$ and $\gamma$ with respect to $\mathrm{x}, \mathrm{y}$ and $z$-axis. A force applied in a direction perpendicular to the line OP. The force $F_{p}$ can be resolved into there respective $\mathrm{x}, \mathrm{y}$ and $\mathrm{z}$ components.

Finding the direction cosine of a line that is perpendicular to the line OP can do this. The direction cosine $\left(\mathrm{l}_{\mathrm{p}}, \mathrm{m}_{\mathrm{p}}\right.$ and $\left.\mathrm{n}_{\mathrm{p}}\right)$ can be found using following steps.

The equation of a plane that is perpendicular to the line OP and passes through the point $(\mathrm{x}, \mathrm{y}, \mathrm{z})$ of the line OP.

$$
1 x_{p}+m y_{p}+n z_{p}=r
$$

$\mathrm{x}_{\mathrm{p}}, \mathrm{y}_{\mathrm{p}}, \mathrm{z}_{\mathrm{p}}$ - Any point on the plane perpendicular to line OP

$1, \mathrm{~m}, \mathrm{n} \quad$ - Direction cosines of the line OP already calculated.

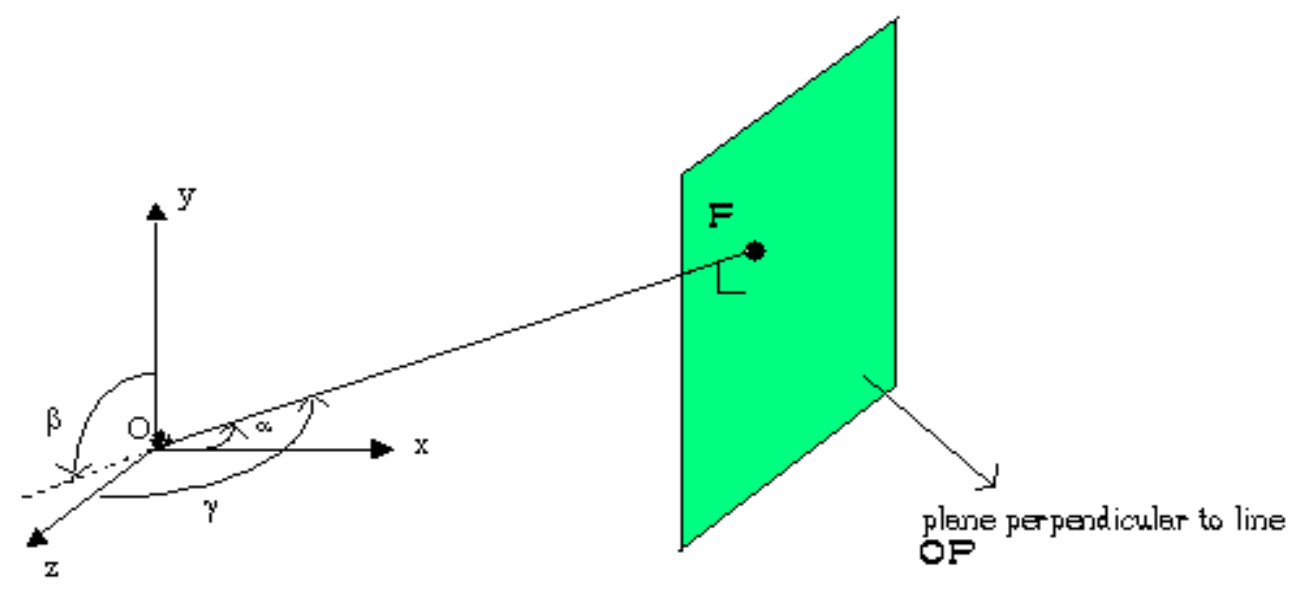


A line on the plane whose equation has been found out, should also be perpendicular to the line OP.

So let

$\mathrm{x}_{\mathrm{p}}=0, \mathrm{y}_{\mathrm{p}}=0$

Then,

$\mathrm{z}_{\mathrm{p}}=\mathrm{r} / \mathrm{n} \quad$ where $\mathrm{n}=\mathrm{z} / \mathrm{r}$

Therefore $\mathbf{z}_{\mathbf{p}}=\mathbf{r}^{2} / \mathbf{z}$

Therefore a line that is perpendicular to the given line OP passes through the point $\left(0,0, \mathrm{r}^{2} / \mathrm{z}\right)$ and $(\mathrm{x}, \mathrm{y}, \mathrm{z})$.

To find the direction cosine of the line passing through the points $\left(0,0, \mathrm{r}^{2} / \mathrm{z}\right)$ and $(\mathrm{x}, \mathrm{y}, \mathrm{z})$ are

$$
r_{p}=\sqrt{(x-0)^{2}+(y-0)^{2}+\left(z-\frac{r^{2}}{z}\right)^{2}}
$$

Therefore the direction cosines of the line perpendicular to the given line OP are

$$
\begin{aligned}
& l_{p}=\frac{x}{r_{p}} \\
& m_{p}=\frac{y}{r_{p}} \\
& n_{p}=\frac{\left(z-\frac{r^{2}}{z}\right)}{r_{p}}
\end{aligned}
$$

Therefore the force $\mathrm{F}_{\mathrm{p}}$ applied in a direction perpendicular to line $\mathrm{OP}$ can be resolved into there respective components as

$$
\begin{aligned}
& \mathbf{F}_{\mathbf{p x}}=\mathbf{l}_{\mathbf{p}} * \mathbf{F}_{\mathbf{p}} \\
& \mathbf{F}_{\mathbf{p y}}=\mathbf{m}_{\mathbf{p}} * \mathbf{F}_{\mathbf{p}} \\
& \mathbf{F}_{\mathbf{p z}}=\mathbf{n}_{\mathbf{p}} * \mathbf{F}_{\mathbf{p}}
\end{aligned}
$$




\section{Appendix 7}

\section{Extraction of load factor, step-increment number and nodal deformation of a particular node for each increment.}

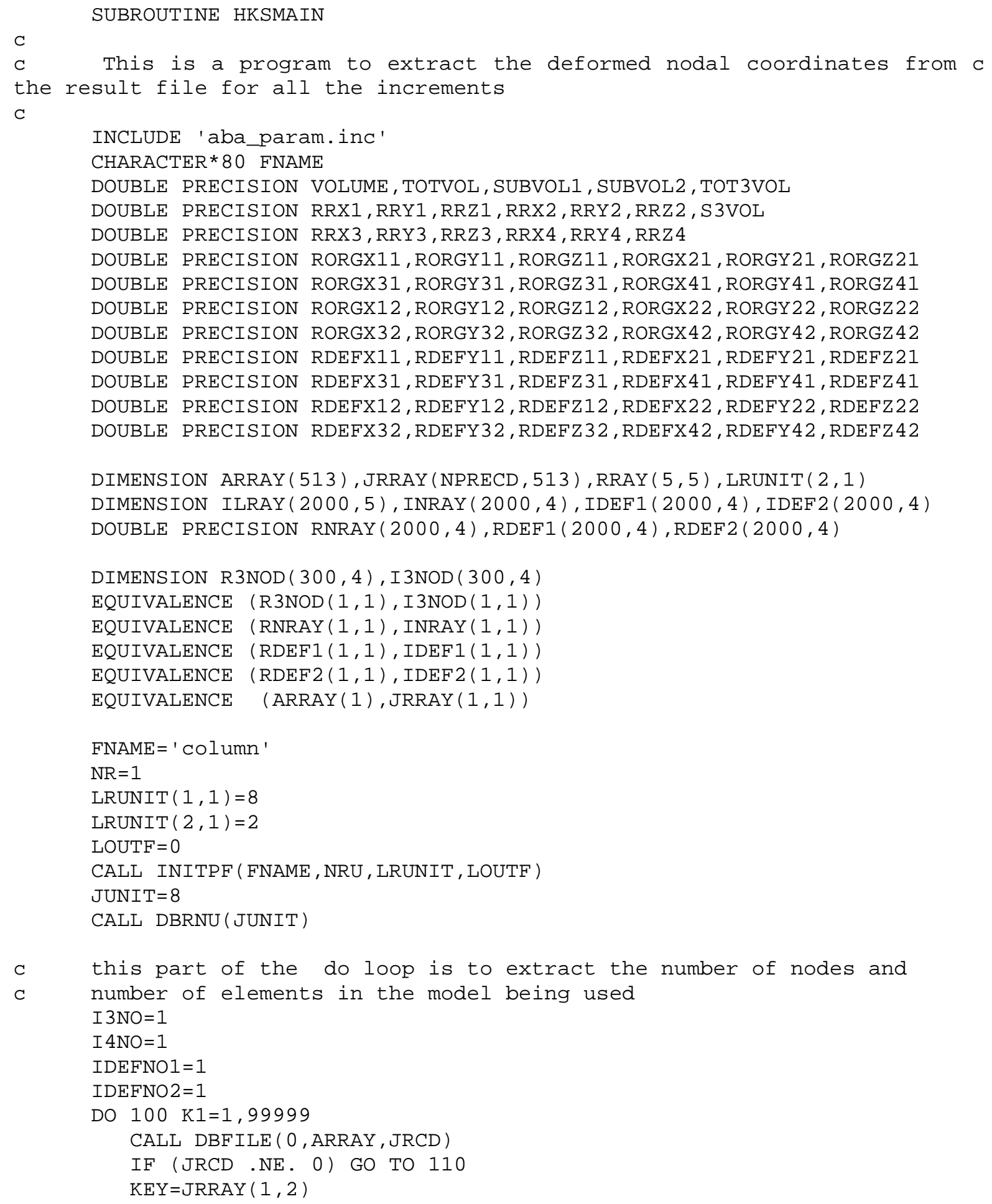


C EXTRACT THE LOAD FACTOR FOR THE INCREMENT AND STORE IN RSTEP

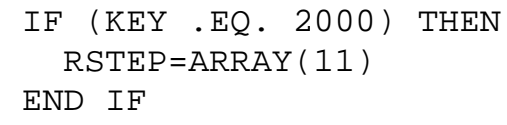

110 CONTINUE STOP

END 


\section{Appendix 8}

Goodier 1933 found a closed form solution for a spherical inclusion inside a medium. The model is shown in the figure. The model is a axis symmetric model of a inclusion inside a infinite medium. This is subjected to a uniform compressive stress $t(\mathrm{~N} / \mathrm{mm})$.

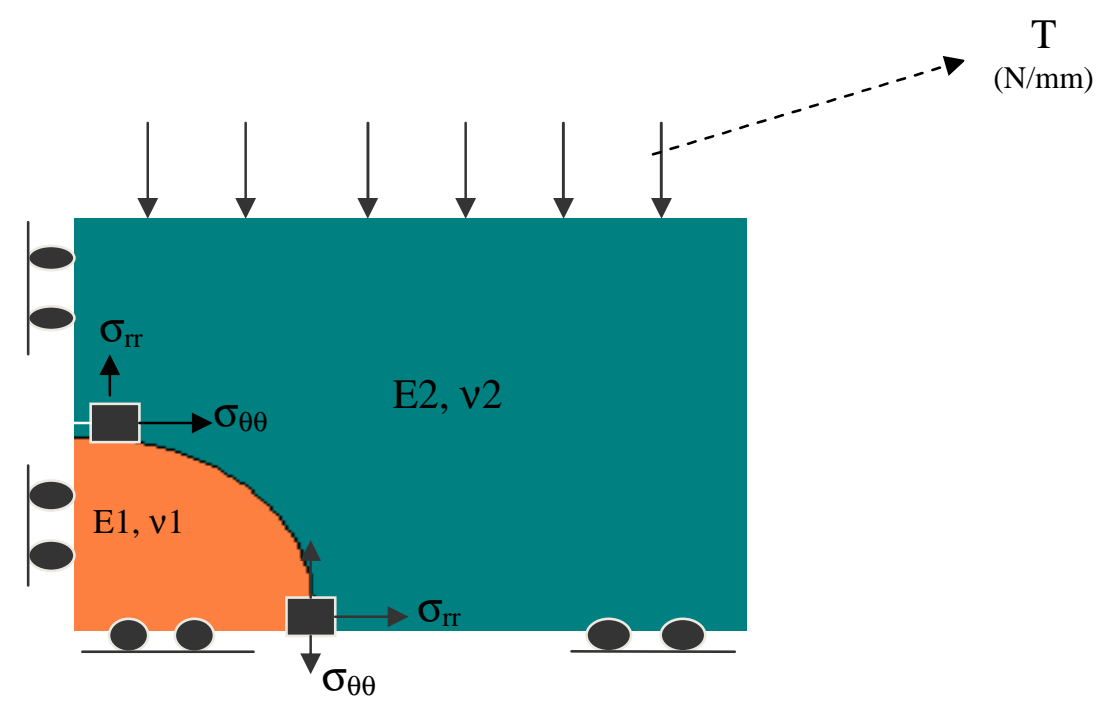

The inclusion is if radius $r$, and material properties E1, $v 1$.

When the material property of the inclusion and the medium is known then the stresses and deformations at any point of the inclusion and the medium can be found by the following set of equations solved by goodier. The radial and tangential deformation at any point on the medium is given by the formula $\mathrm{U}_{\mathrm{r}}^{\mathrm{b}}$ and $\mathrm{U}_{\theta}^{\mathrm{b}}$. There are separate set of equations for finding the radial and tangential deformations for any point inside the inclusion. But at the juction between the inclusion and the medium, the deformation equations for both medium and the inclusion gives the same results.

$$
\begin{gathered}
U_{r}^{b}=-\frac{A}{r^{2}}-\frac{3 B}{r^{4}}+\left(\frac{5-4 v_{b}}{1-2 v_{b}}\right) \frac{2 C}{3 r^{3}}+\left[\frac{-9 B}{r^{4}}+\left(\frac{5-4 v_{b}}{1-2 v_{b}}\right) \frac{C}{r^{2}}\right] \cos 2 \theta+\frac{T r}{2 E}\left[\left(1-v_{b}\right)+\left(1+v_{b}\right) \cos 2 \theta\right] \\
U_{\theta}^{b}=-\left[\frac{6 B}{r^{4}}+\frac{2 C}{r^{2}}\right] \sin (2 \theta)-\frac{T r}{2 E}\left(1+v_{b}\right) \sin 2 \theta
\end{gathered}
$$




$$
\begin{gathered}
A=\frac{T a^{3}\left[\left(-1-v_{b}+2 v_{t}\right) \mu_{b}+\left(1-2 v_{b}+v_{t}\right) \mu_{t}+2 v_{b} v_{t}\left(\mu_{b}-\mu_{t}\right)\right]}{6 \mu_{b}\left(1+v_{b}\right)\left[\left(2-4 v_{t}\right) \mu_{b}+\left(1+v_{t}\right) \mu_{t}\right]} \\
B=\frac{\left.T a^{5}\left(\mu_{b}-\mu_{t}\right)\right]}{8 \mu_{b}\left[\left(7-5 v_{b}\right) \mu_{b}+\left(8-10 v_{b}\right) \mu_{t}\right]} \\
C=\frac{\left.5 T a^{3}\left(1-2 v_{b}\right)\left(\mu_{b}-\mu_{t}\right)\right]}{8 \mu_{b}\left[\left(7-5 v_{b}\right) \mu_{b}+\left(8-10 v_{b}\right) \mu_{t}\right]}
\end{gathered}
$$

Where

a - location on the model where the deformation are needed to be found.

Any formulae with a sub-script "b" is related to the medium property and sub-script " $\mathrm{t}$ " belongs to the inclusion property.

So from the material properties of the medium and inclusion, and their dimensions, we can calculate the values for the function A, B, C. From this we can arrive at the deformations and stress at any point in the medium including the boundary between the inclusion and the medium. While the deformation and stresses at any point for the inclusion are found using a different set of functions. 


\section{Appendix 9}

\section{ABAQUS subroutine for extracting the nodel deformed coordinates from the .fil file and calcuate the change in volume and hence its bulk modulus.}

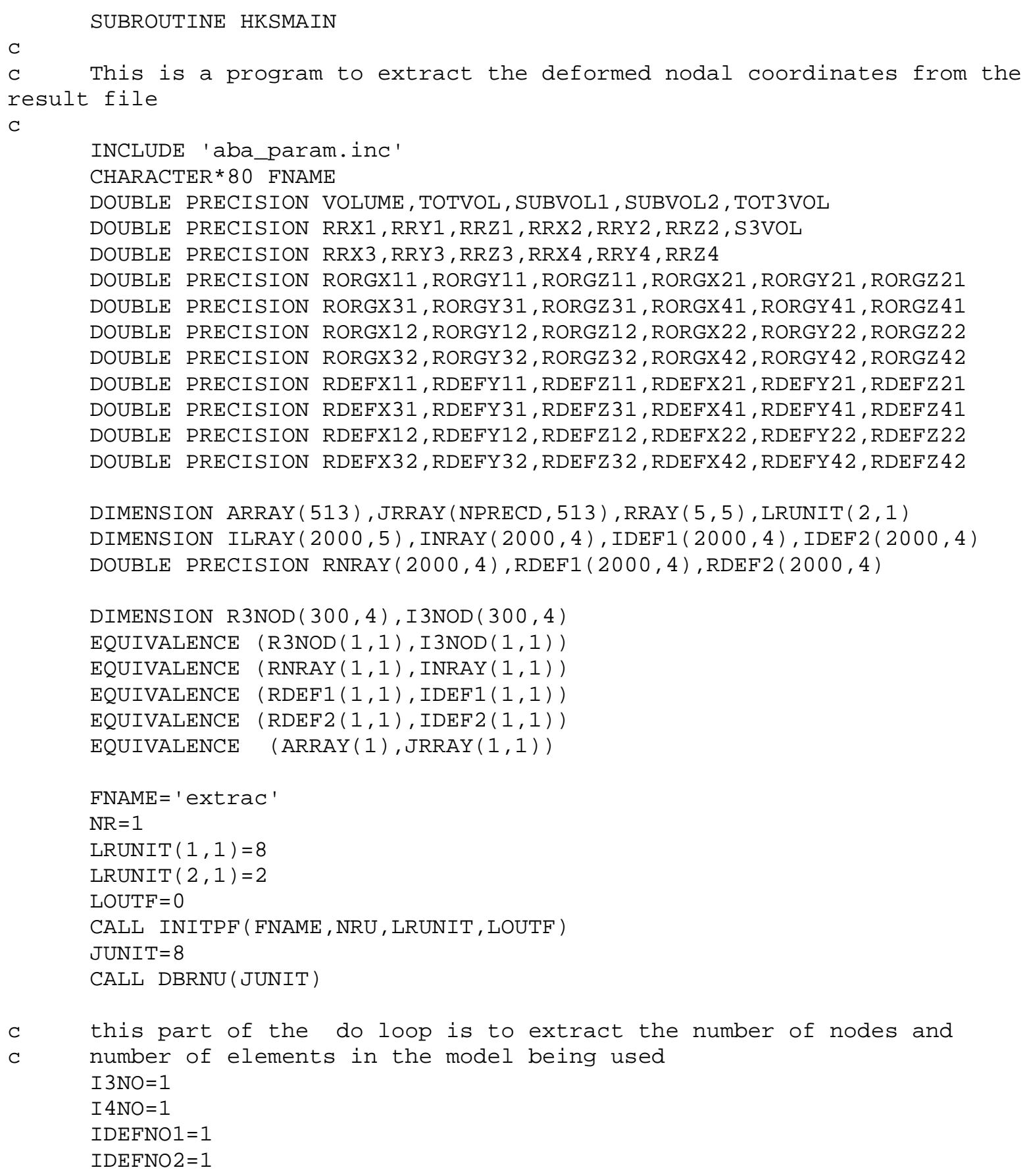




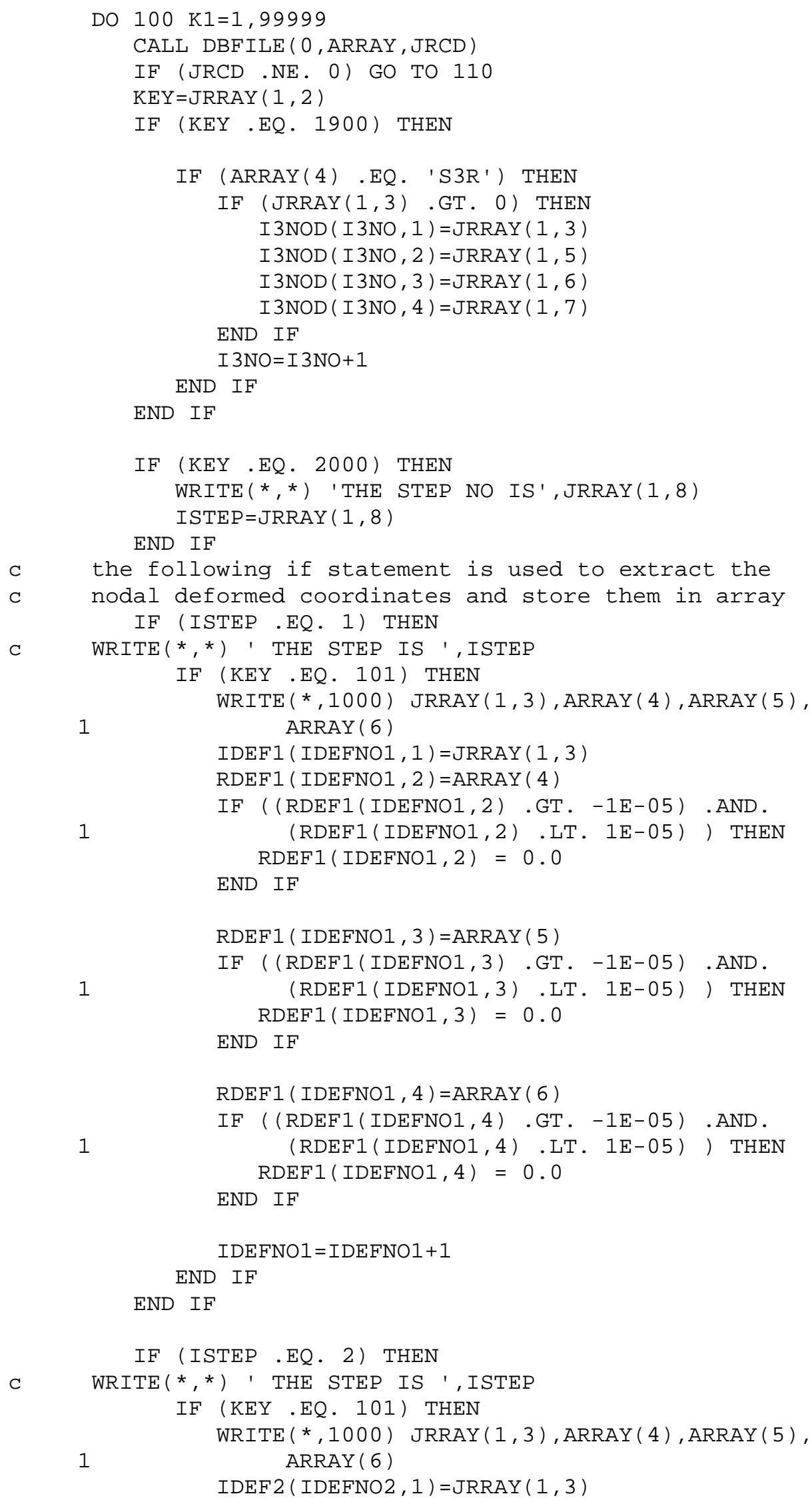




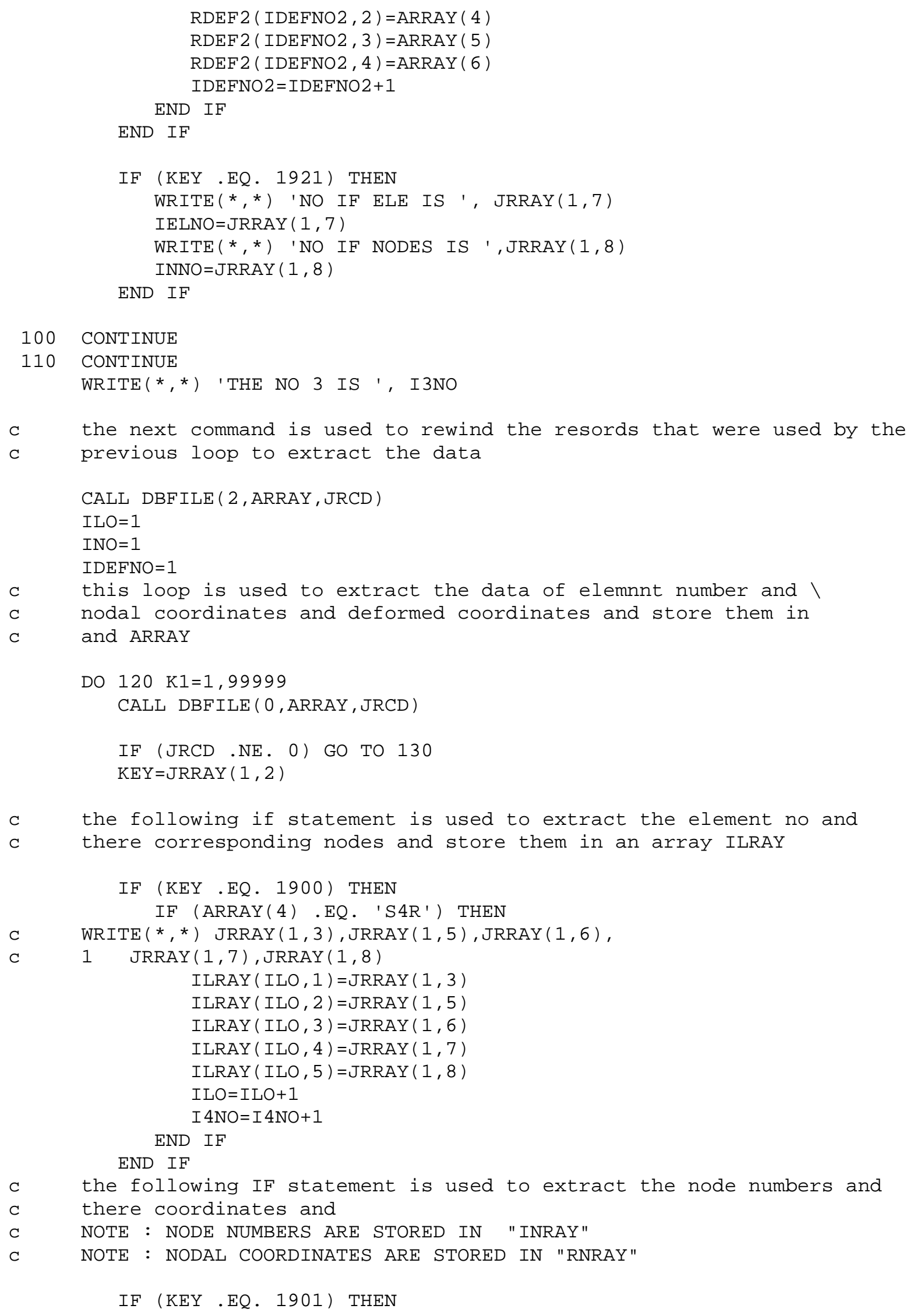




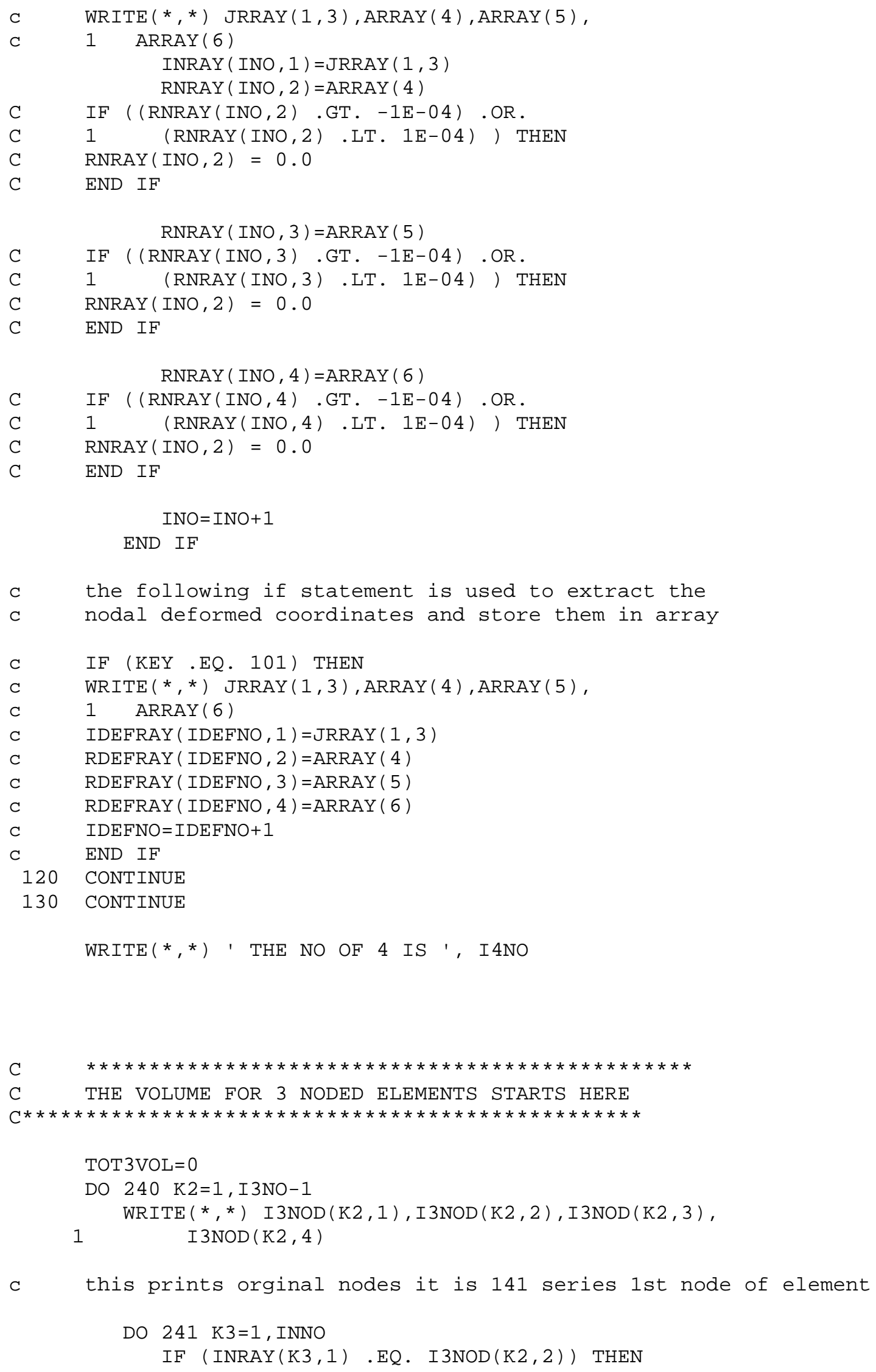




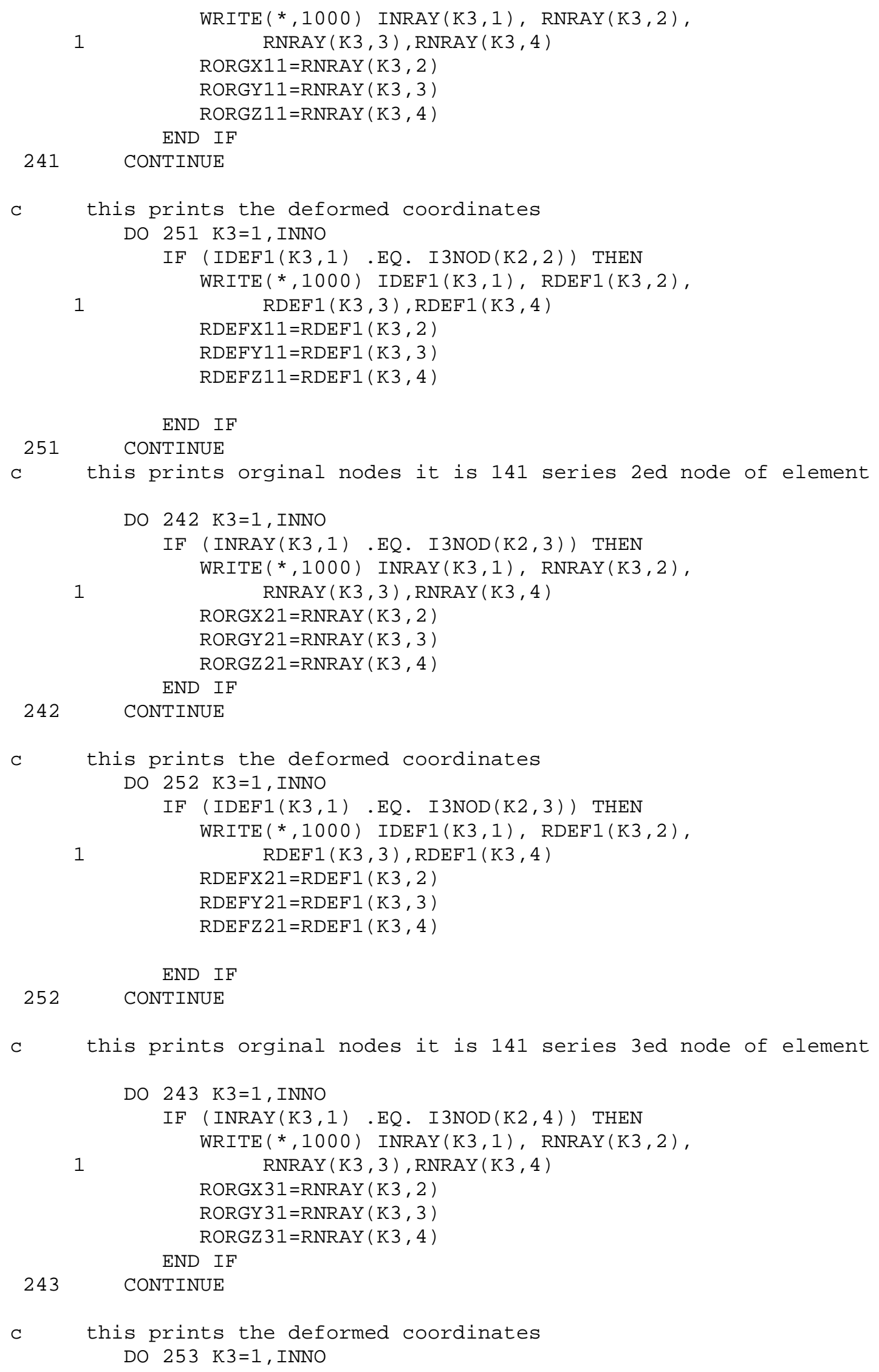




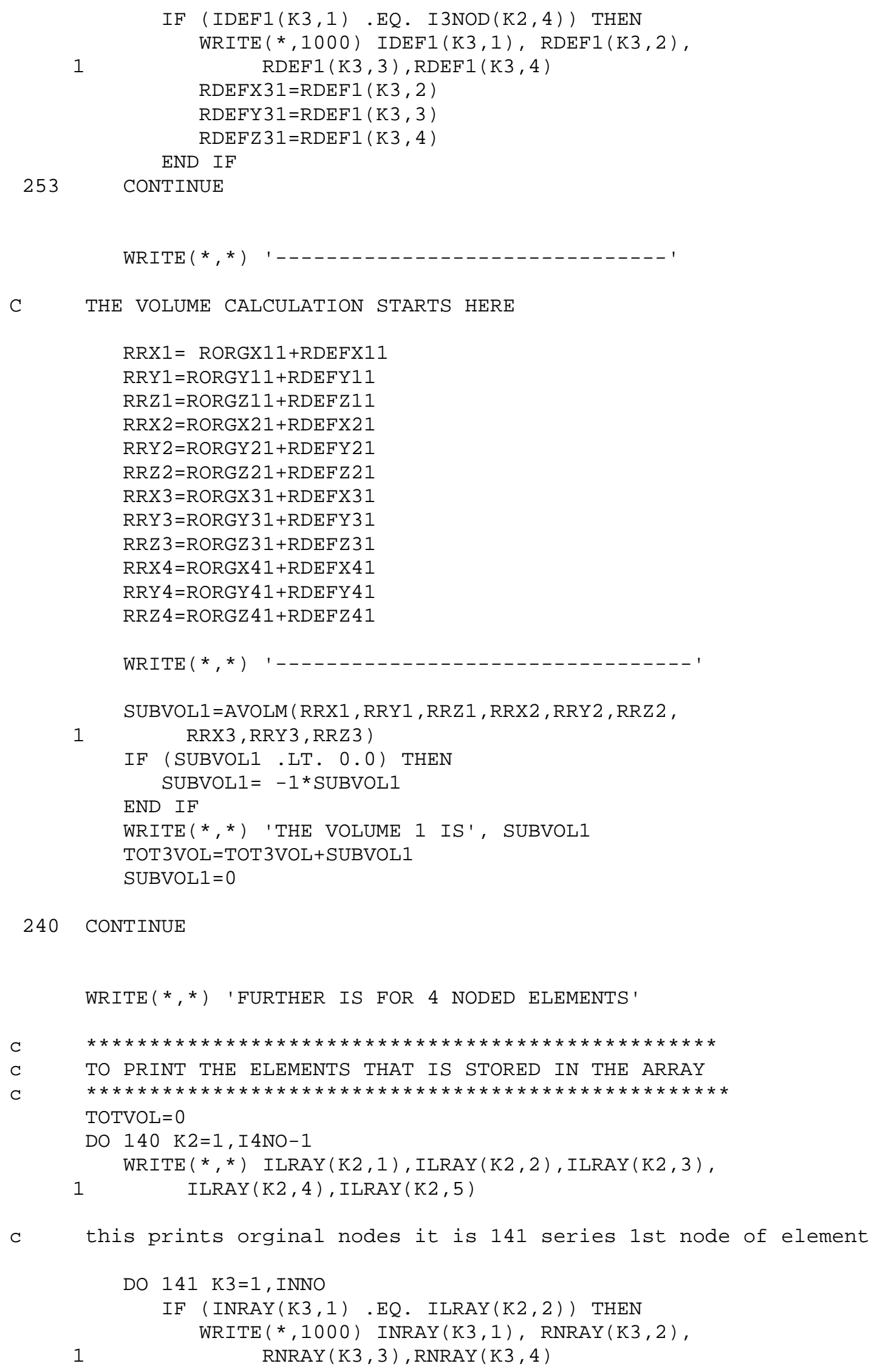




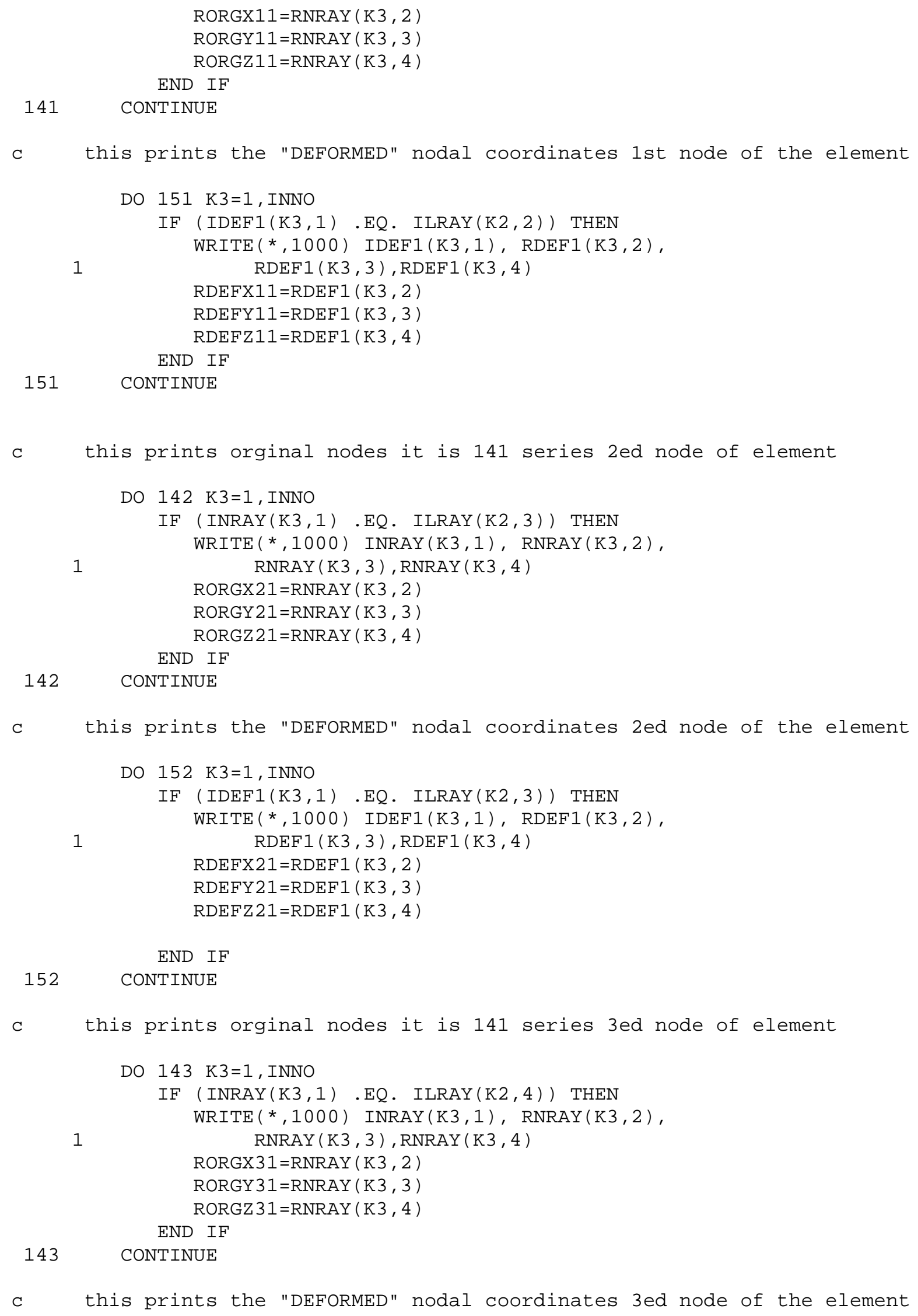




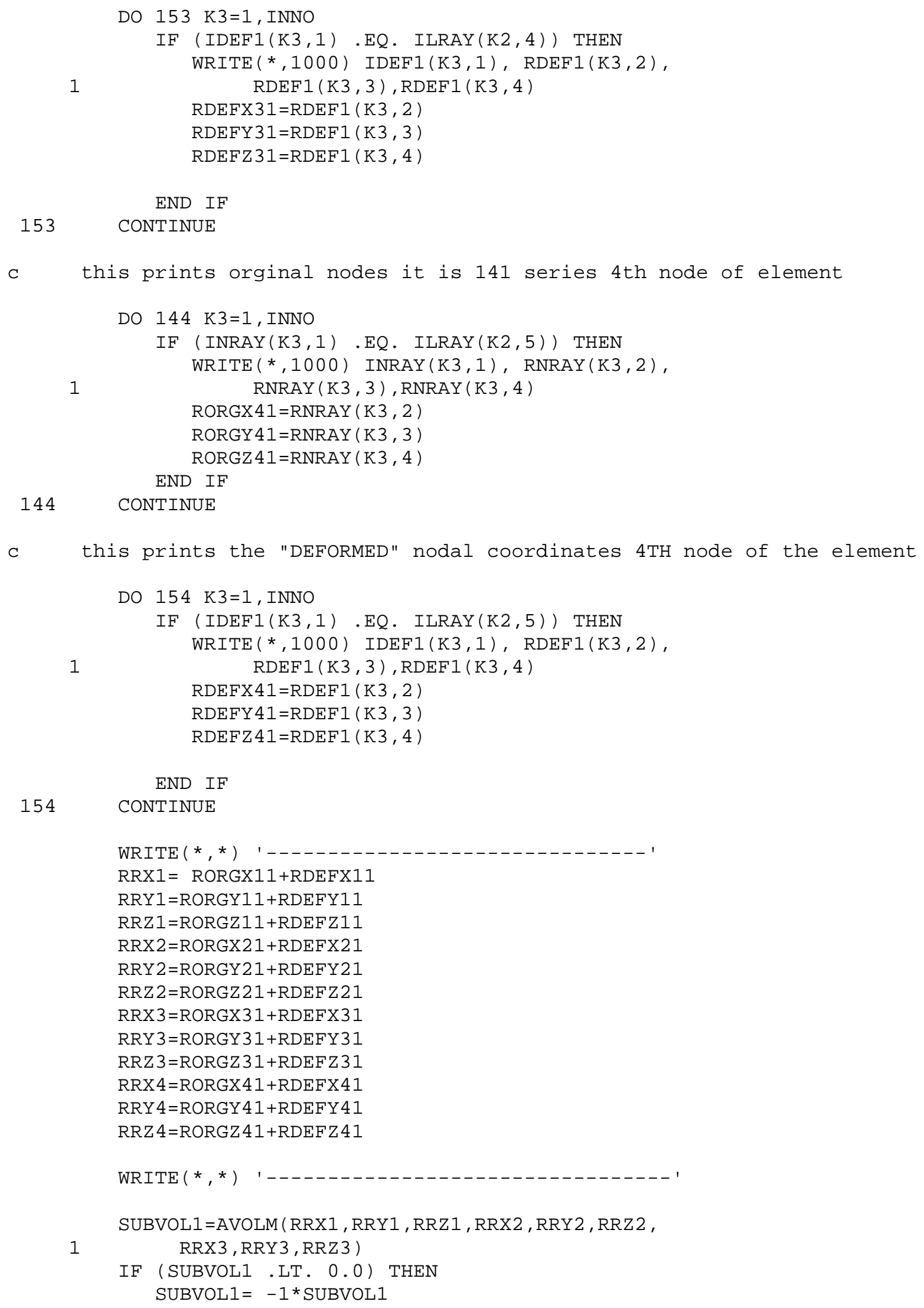

C

this prints the "DEFORMED" nodal coordinates $4 \mathrm{TH}$ node of the element

DO $154 \mathrm{~K} 3=1$, INNO

$\operatorname{IF}(\operatorname{IDEF} 1(\mathrm{~K} 3,1) \cdot \mathrm{EQ} \cdot \operatorname{ILRAY}(\mathrm{K} 2,5))$ THEN

$\operatorname{WRITE}(*, 1000) \operatorname{IDEF} 1(\mathrm{~K} 3,1), \operatorname{RDEF} 1(\mathrm{~K} 3,2)$,

1 $\operatorname{RDEF} 1(\mathrm{~K} 3,3), \operatorname{RDEF} 1(\mathrm{~K} 3,4)$ 


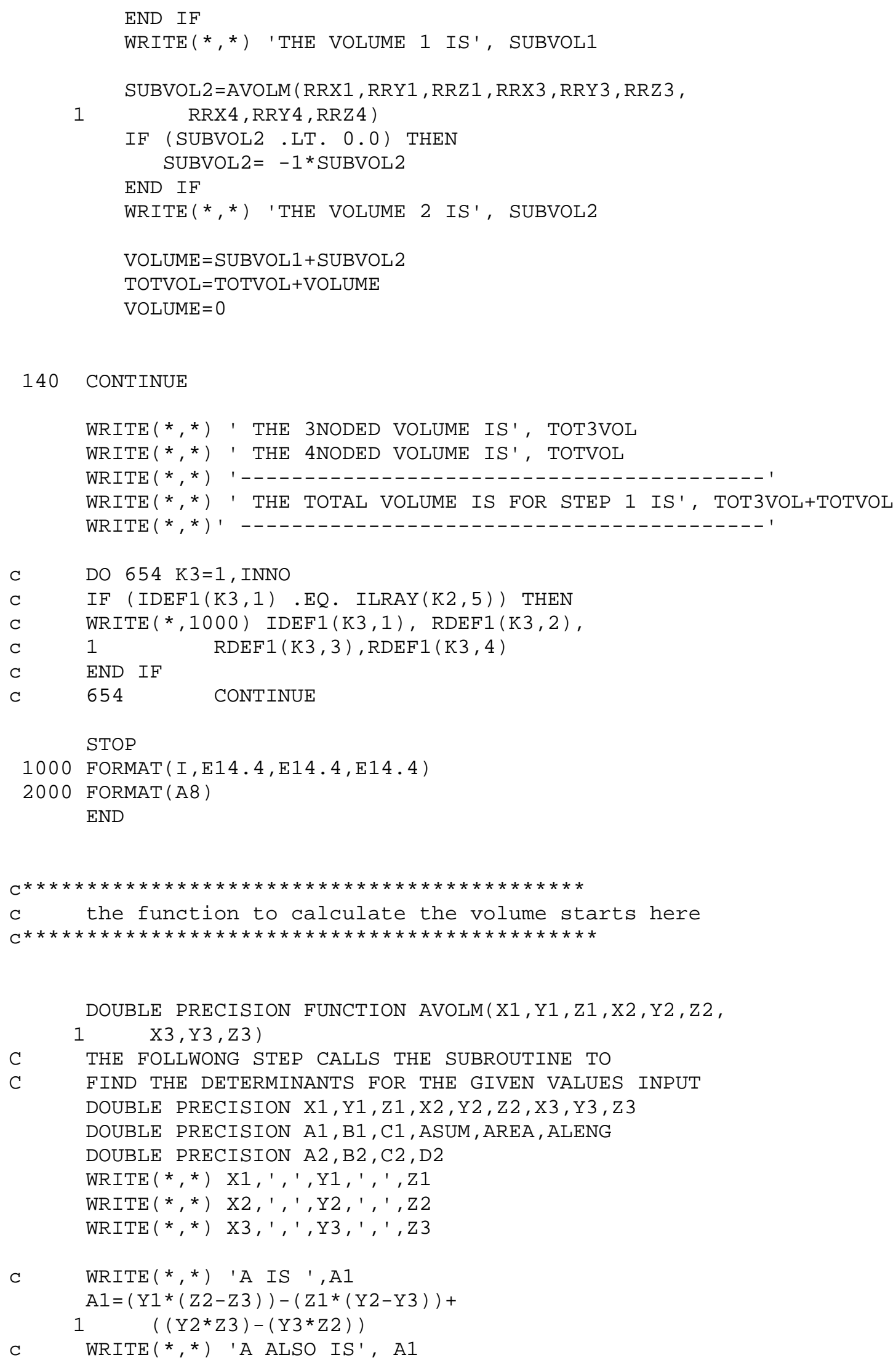


C THE FOLLWONG STEP CALLS THE SUBROUTINE TO

C FIND THE DETERMINANTS FOR THE GIVEN VALUES INPUT

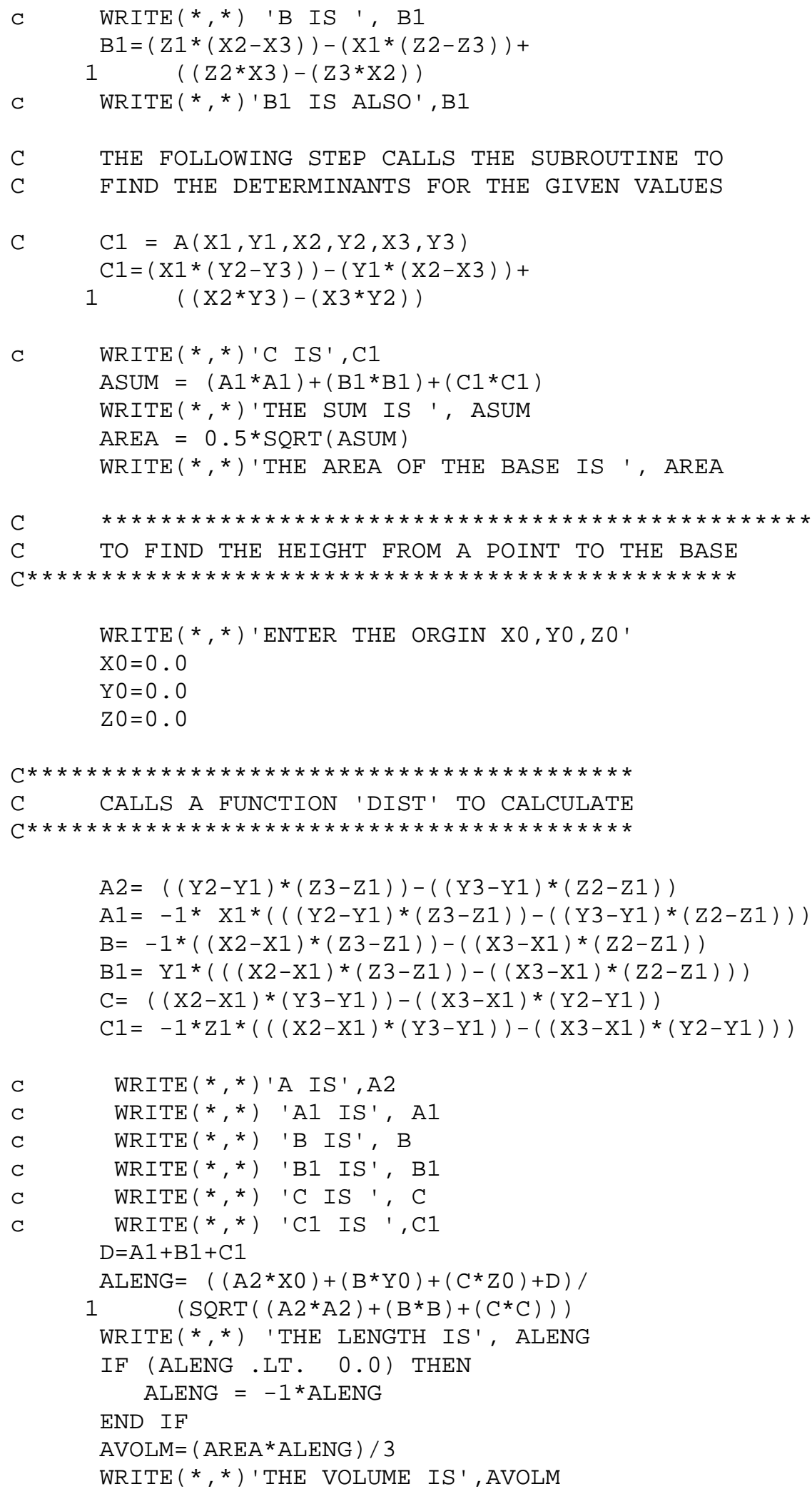


END

C-----------------------------------------------

C THE FUNCTIONS FOR CALCULATING DISTANCE STARTS HERE

DOUBLE PRECISION FUNCTION DIST (AX1, AX2, AX3, BX1, BX2, BX3)

DOUBLE PRECISION AX1, AX2, BX1, BX2, BX3, AX3

C $\quad \operatorname{WRITE}(*, *) \mathrm{AX} 1, \mathrm{AX} 2, \mathrm{AX} 3, \mathrm{BX} 1, \mathrm{BX} 2, \mathrm{BX} 3$

$\mathrm{DIST}=((\mathrm{AX} 2-\mathrm{AX} 1) *(\mathrm{BX} 3-\mathrm{BX} 1))-((\mathrm{BX} 2-\mathrm{BX} 1) *(\mathrm{AX} 3-\mathrm{AX} 1))$

END 